\title{
Intermolecular Cross-Double-Michael Addition between Nitro and Carbonyl Activated Olefins as A New Approach in C-C Bond Formation
}

\author{
Xiaohua Sun, ${ }^{\text {a }}$ Sujata Sengupta, ${ }^{\text {a }}$ Jeffrey L. Petersen, ${ }^{\text {a }}$ Hong \\ Wang, ${ }^{\mathrm{b}}$ James Lewis, ${ }^{\mathrm{b}}$ Xiaodong Shi ${ }^{\text {a }}$
}

C. Eugene Bennett Department of Chemistry ${ }^{\mathrm{a}} \&$ Department of Physics ${ }^{\mathrm{b}}$ at West Virginia University, Morgantown, WV 26506, USA

\section{Email:Xiaodong.Shi@mail.wvu.edu}

$\begin{array}{lll}\text { I. } & \text { General Methods and Materials } & \text { S2-S3 } \\ \text { II. } & \text { NMR Spectra of the Reaction between 1a and 2a to Form 3 } & \text { S4 } \\ \text { III. } & \text { Compounds Characterization } & \text { S5-S13 } \\ \text { IV. } & \text { The Computation Studies } & \text { S14 } \\ \text { V. } & \text { ORTEP Drawing of the crystal structures } & \text { S15-S16 } \\ \text { VI. } & { }^{1} \text { H-NMR and }{ }^{13} \text { C-NMR Spectra } & \text { S17-S64 }\end{array}$ 


\section{General Methods and materials:}

Unless otherwise noted, all commercial reagents and solvents were obtained from the commercial provider and used without further purification. Air and/or moisture-sensitive reactions were carried out under an atmosphere of nitrogen using oven/flame-dried glassware and standard syringe/septa techniques. ${ }^{1} \mathrm{H}-\mathrm{NMR}$ and ${ }^{13} \mathrm{C}-\mathrm{NMR}$ spectra were recorded on Joel 270 and Varian $600 \mathrm{MHz}$ spectrometers. Chemical shifts were reported relative to internal tetramethylsilane $(\delta 0.00 \mathrm{ppm})$ or $\mathrm{CDCl}_{3}(\delta 7.26 \mathrm{ppm})$ or DMSO $(\delta 2.49 \mathrm{ppm})$ for ${ }^{1} \mathrm{H}$ and $\mathrm{CDCl}_{3}$ $(\delta 77.0 \mathrm{ppm}$ ) or DMSO ( $\delta 39.5 \mathrm{ppm})$ for ${ }^{13} \mathrm{C}$. Infrared (IR) spectra were obtained on a Prospect MIDAC FT-IR spectrometer. Flash column chromatography was performed on 230-430 mesh silica gel. Analytical thin layer chromatography was performed with precoated glass baked plates $(250 \mu)$ and visualized by fluorescence and by charring after treatment with potassium permanganate stain. $\mathrm{R}_{f}$ values were obtained by elution in the stated solvent ratios.

General Procedure for Intermolecular tandem double Michael addition reaction: The nitroalkene (1.0 eq.) was added to a solution of $\alpha, \beta$-unsaturated ketone/ester (2.0 eq.), L-Proline (20 mol\%) and $\mathrm{NaN}_{3}$ (1.0 eq.) in DMSO. The resulting reaction mixture was stirred at room temperature for $5 \mathrm{hrs}$ followed by the dilution with dichloromethane. The organic layer was washed with water and brine. The combined organic layer was dried over anhydrous sodium sulfate. The solvent was removed under reduced pressure with flash silica gel chromatography to give the pure product.

Synthesis of compound 6a: The zinc powder (440 mg, $6.76 \mathrm{mmols})$ was added into the solution of $\mathbf{5 f}(720 \mathrm{mg}, 3.38 \mathrm{mmols})$ in acetic acid $(2.5 \mathrm{~mL})$ over 10 minutes at $0{ }^{\circ} \mathrm{C}$. The resuling reaction mixture was stirred at room temperature for $1.5 \mathrm{hr}$. followed by the filtration through Celite with washing by ether $(20 \mathrm{~mL})$. The filtrate was then diluted with water and cooled to 0 ${ }^{\circ} \mathrm{C}$. The $10 \mathrm{M}$ sodium hydroxide solution was added until the aqueous layer $\mathrm{pH}$ was 12 . After separation, the aqueous phase was extracted with ether $(2 \times 50 \mathrm{~mL})$. The combined organic layer was washed with water $(50 \mathrm{~mL})$, dried over sodium sulfate and removed by vacuum. Purification by column chromatography gave the desired amine $\mathbf{6 a}$. 
Synthesis of allylic alcohol 6b: Compound $5 \mathbf{j}$ (175 mg, 1.0 eq.) was dissolved in 1,2dichlorobenzene to which was added DABCO (56 mg, 1.0 eq.). The resulting reaction mixture was then refluxed at $180{ }^{\circ} \mathrm{C}$ for 3 hrs. The crude product was purified by flash chromatography (Hex/EA 8:2) to give $131 \mathrm{mg}$ of $\mathbf{6 b}$ as a pale red oil an exclusively as a single diastereomer.

General procedure for Nef reaction for synthesis of compound 6c: To a solution of compound 5q $(227 \mathrm{mg}, 1.0 \mathrm{mmols})$ in methanol was added sodium methoxide $(25 \mathrm{mg})$ in methanol at $0{ }^{\circ} \mathrm{C}$ and the mixture was stirred for $0.5 \mathrm{hrs}$. To the nitronate solution was added a mixture of conc. sulfuric acid $(0.2 \mathrm{~mL})$ and methanol $(10 \mathrm{~mL})$. During the addition, the mixture was kept at $0{ }^{\circ} \mathrm{C}$ and after additional stirring at $0{ }^{\circ} \mathrm{C}$ for an hour, it was poured into dichloromethane $(20 \mathrm{~mL})$.The organic layer was washed with cold water and the water layer was extracted with dichloromethane. The combined organic layer was dried over sodium sulfate and the solvent was removed under vacuum pressure. Dichloromethane and $2 \mathrm{~N}$ hydrochloric acid was added and the mixture was stirred at room temperature for $2 \mathrm{hrs}$. The water layer was then washed with dichloromethane and the organic layer was dried over sodium sulfate and removed under vacuum and the crude product was then purified by column chromatography to give $\mathbf{6 c}$ in $72 \%$ yield.

Preparation of single crystal for X-ray diffraction: Single crystal suitable for X-ray diffraction analysis was obtained by slow evaporation of the solution of $\mathbf{5 d}$ and $\mathbf{6 a}$ in acetone. 


\section{NMR spectra of the reaction between 1a and 2a forming 3.}

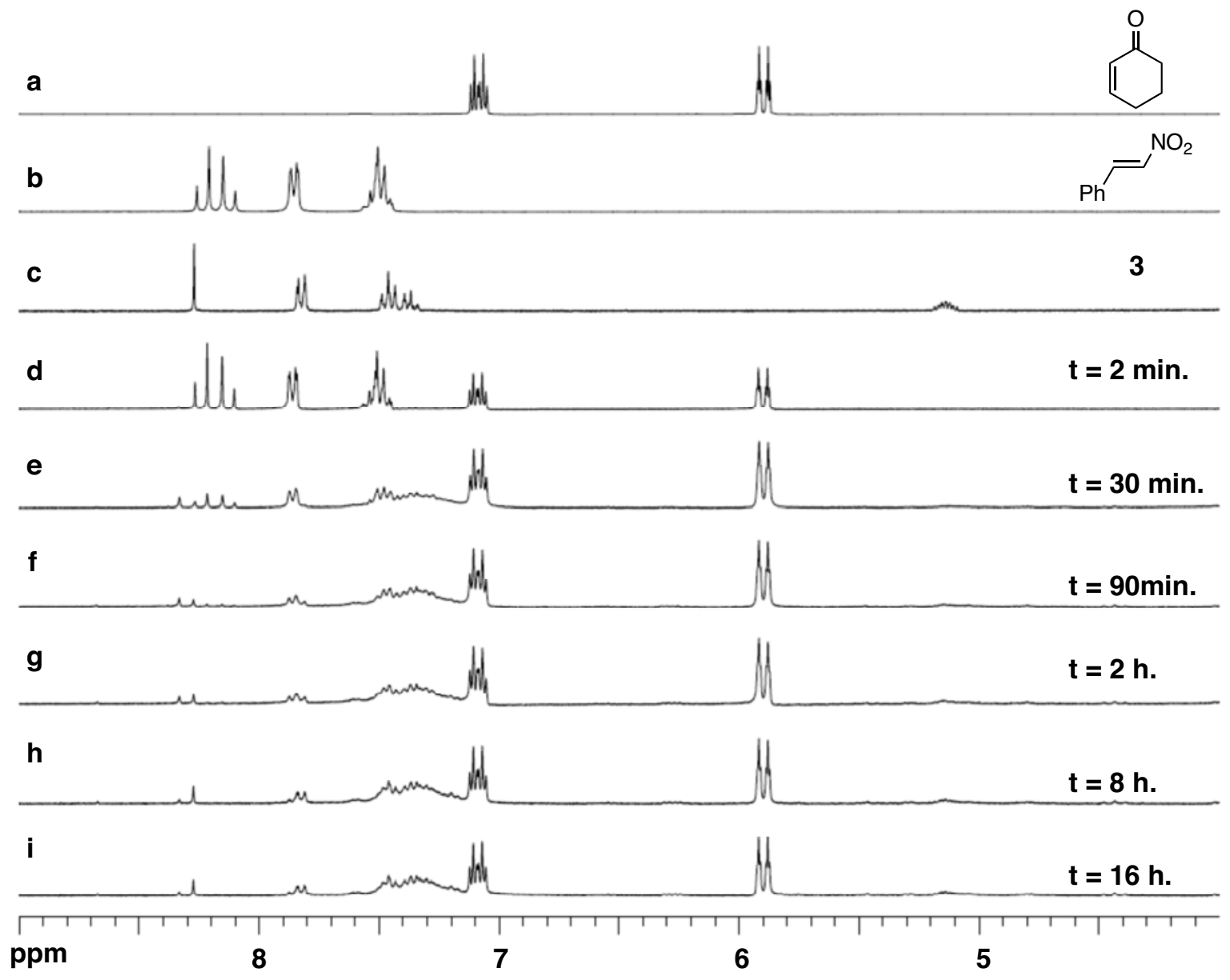

Figure S-1. The NMR spectra of the reaction between $1 \mathbf{a}$ and $\mathbf{2 a}$ : a) 2a; b) 1a; c) 3, a mixture of two double bond isomers with a ration of $2: 1$; d) reaction between $1 \mathrm{a}$ and 2 a when time is 2 min.; e) 30 min.; f) 90 min.; g) 2 h.; h) 8 h.; i) 2 h.

Based on the spectra, the nitroalkene was consumed rapidly. At 30 minutes, more than 90\% 1a was consumed while no 3 forming. The amount of $\mathbf{3}$ kept increasing even almost all 1a was consumed. These spectra clearly revealed two important mechanistic facts: 1) nitroalkene is more reactive Michael receptor than enone; and 2) the oligomerization of 1a is equilibrium and can come back to simple nitroalkene before polymerization. This result set up our key hypothesis that installation of a $\beta$-alkyl group will help the reaction to reach the desired cross double Michael addition. 


\section{Compound Characterization:}<smiles>C=C(c1ccccc1)C(C1CCCC(=O)C1)[N+](=O)[O-]</smiles>

(S)-3-((R)-1-nitro-2-phenylallyl) cyclohexanone (d:r = 1:1) (5a): 5a was purified by flash chromatography (Hexane-EtOAc, v/v 5/1) as yellow oil with overall isolated yield: 87\%. The two diastereomer were isolated. Isomer 1: ${ }^{1} \mathrm{H}-\mathrm{NMR}\left(270 \mathrm{MHz}, \mathrm{CDCl}_{3}\right) \delta 1.37-1.82(\mathrm{~m}, 2 \mathrm{H})$, $1.82-1.98(\mathrm{~m}, 2 \mathrm{H}), 2.00-2.26(\mathrm{~m}, 2 \mathrm{H}), 2.32-2.52(\mathrm{~m}, 2 \mathrm{H}), 2.60-2.82(\mathrm{~m}, 1 \mathrm{H}), 5.24(\mathrm{~d}, J=10.4$ $\mathrm{Hz}, 1 \mathrm{H}), 5.65(\mathrm{~s}, 1 \mathrm{H}), 5.71(\mathrm{~s}, 1 \mathrm{H}), 7.30-7.40(\mathrm{~m}, 5 \mathrm{H}) ;{ }^{13} \mathrm{C}-\mathrm{NMR}\left(67.5 \mathrm{MHz}, \mathrm{CDCl}_{3}\right) \delta 24.1$, 28.5, 41.0, 42.3, 43.2, 94.1, 119.3, 126.6, 128.7, 129.0, 139.5, 141.7, 208.5; IR (cm¹) : 2940, 2346, 1708, 1547, 1446; HRMS Calculated for $\left[\mathrm{C}_{15} \mathrm{H}_{17} \mathrm{NO}_{3} \mathrm{Na}\right]^{+}: 282.1106$, Found: 282.1101.

Isomer 2: ${ }^{1} \mathrm{H}-\mathrm{NMR}\left(270 \mathrm{MHz}, \mathrm{CDCl}_{3}\right) \delta 1.52-2.53(\mathrm{~m}, 8 \mathrm{H}), 2.81-3.02(\mathrm{~m}, 1 \mathrm{H}), 5.27(\mathrm{~d}, J=7.4$ $\mathrm{Hz}, 1 \mathrm{H}), 5.62(\mathrm{~s}, 1 \mathrm{H}), 5.67(\mathrm{~s}, 1 \mathrm{H}), 7.20-7.45(\mathrm{~m}, 5 \mathrm{H}){ }^{13} \mathrm{C}-\mathrm{NMR}\left(67.5 \mathrm{MHz}, \mathrm{CDCl}_{3}\right) \delta 24.3,27.1$, $40.9,42.0,44.4,94.1,118.9,126.6,128.7,129.0,139.5,139.5,208.2$.<smiles>C=C(c1ccccc1)C(C1CCCCC(=O)C1)[N+](=O)[O-]</smiles>

3-(1-nitro-2-phenylallyl)cycloheptanone $(\mathrm{d}: \mathrm{r}=1.2: 1)(\mathbf{5 b}): \mathbf{5 b}$ was purified by flash chromatography (Hexane-EtOAc, v/v 5/1) as yellow oil with overall isolated yield: 72\%. The two diastereomers cannot be separated. Two sets of signals were obtained for the ${ }^{1} \mathrm{H}$ and ${ }^{13} \mathrm{C}$ NMR. ${ }^{1} \mathrm{H}-\mathrm{NMR}\left(270 \mathrm{MHz}, \mathrm{CDCl}_{3}\right) \delta$ 1.35-1.64 (m, 6H), 1.85-1.92 (m, 4H), 2.1-2.2 (m, 4H), 2.48-2.58 (m, 8H), $5.21(\mathrm{~d}, \mathrm{~J}=9.7 \mathrm{~Hz}, 1 \mathrm{H}), 5.26(\mathrm{~d}, \mathrm{~J}=8.9 \mathrm{~Hz}, 1 \mathrm{H}), 5.64(\mathrm{~s}, 1 \mathrm{H}), 5.67(\mathrm{~s}, 1 \mathrm{H}), 5.70$ (s, 1H), $5.75(\mathrm{~s}, 1 \mathrm{H}), 7.33-7.40(\mathrm{~m}, 5 \mathrm{H}) ;{ }^{13} \mathrm{C}-\mathrm{NMR}\left(67.5 \mathrm{MHz}, \mathrm{CDCl}_{3}\right) \delta 24.3,24.4,28.2,28.3$, $32.0,33.4,39.2,39.7,43.5,45.0,46.0,94.1,94.3,119.0,119.4,126.6,126.7,128.7$ (overlapped), 128.9 (overlapped), 139.3, 139.4, 142.3, 142.5, 211.4, 211.5; IR (cm¹): 2944, 2355, 1698 1542, 1438; HRMS Calculated for $\left[\mathrm{C}_{16} \mathrm{H}_{19} \mathrm{NO}_{3} \mathrm{Na}\right]^{+}:$296.12626, Found: 296.12576. 
<smiles>C=C(c1ccccc1)C(C1CCC(=O)C1)[N+](=O)[O-]</smiles>

3-(1-nitro-2-phenylallyl) cyclopentanone (d:r $=1.1: 1)(5 \mathrm{c}): 5 \mathrm{c}$ was purified by flash chromatography (Hexane-EtOAc, v/v 3/1) as yellow oil, isolated yield 55\%. The two diastereomers cannot be separated. Two sets of signals were obtained for the ${ }^{1} \mathrm{H}$ and ${ }^{13} \mathrm{C}$ NMR. ${ }^{1} \mathrm{H}-\mathrm{NMR}\left(270 \mathrm{MHz}, \mathrm{CDCl}_{3}\right): \delta$ 1.20-1.82 (m, 4H), 2.03-2.62 (m, 10H), 3.05-3.22 (m, 2H), 5.27 $(\mathrm{d}, \mathrm{J}=10.3 \mathrm{~Hz}, 2 \mathrm{H}), 5.62(\mathrm{~s}, 1 \mathrm{H}), 5.67(\mathrm{~s}, 1 \mathrm{H}), 5.69(\mathrm{~s}, 1 \mathrm{H}), 5.74(\mathrm{~s}, 1 \mathrm{H}), 7.34-7.65(\mathrm{~m}, 10 \mathrm{H}) ;{ }^{13} \mathrm{C}-$ NMR (67.5 MHz, $\left.\mathrm{CDCl}_{3}\right): \delta$ 25.8, 27.3, 37.9, 38.1, 40.1, 40.2, 41.3, 42.8, 94.1, 94.2, 119.5, 119.7, 126.7, 126.8, 128.7 (overlapped), 128.9 (overlapped), 138.9, 139.0, 142.5, 142.8, 215.4, 215.5; IR (cm-1): 2195, 1690,1546, 1497, 1436; HRMS calculated for $\left[\mathrm{C}_{14} \mathrm{H}_{15} \mathrm{NO}_{3} \mathrm{Na}^{+}\right.$: 268.0949, found 286.09486<smiles>C=C(c1ccccc1)C(CC(=O)c1ccccc1)c1ccccc1</smiles>

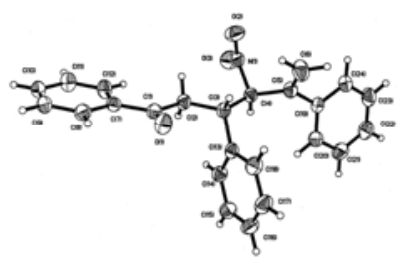

Crystal structure of anti isomer

(3S, 4R)-4-nitro-1,3,5-triphenylhex-5-en-one $(\mathrm{d}: \mathrm{r}=1.6: 1)(\mathbf{5 d}): \mathbf{5 d}$ was purified by flash chromatography (Hexane-EtOAc, v/v 3/1) as White solid, isolated yield: 70\%, m.p. $165^{\circ} \mathrm{C}$. The two diastereomers cannot be separated by column chromatography. The purification was achieved by recrystallization of anti-isomer. IR (cm-1): 2175, 1674,1534, 1497, 1446; HRMS Calculated for $\left[\mathrm{C}_{24} \mathrm{H}_{21} \mathrm{NO}_{3} \mathrm{Na}\right]^{+}: 394.1419$, Found: 394.1415 .

For anti isomer, ${ }^{1} \mathrm{H}-\mathrm{NMR}\left(270 \mathrm{MHz}, \mathrm{CDCl}_{3}\right) \delta 3.22(\mathrm{dd}, J=16.8,3.5 \mathrm{~Hz}, 1 \mathrm{H}), 3.21(\mathrm{dd}, J=$ 17.1, 9.6 Hz, 1H), 4.44 (ddd, $J=16.8,9.9,3.5 \mathrm{~Hz}, 1 \mathrm{H}), 5.75$ (s, 1H), 5.89 (d, J=11.6 Hz, 1H), $6.01(\mathrm{~s}, 1 \mathrm{H}), 7.12-7.57(\mathrm{~m}, 13 \mathrm{H}), 7.66-7.69(\mathrm{~m}, 2 \mathrm{H}) ;{ }^{13} \mathrm{C}-\mathrm{NMR}\left(67.5 \mathrm{MHz}, \mathrm{CDCl}_{3}\right) \delta 41.1,45.2$, 93.9, 120.1, 126.7, 127.9, 128.0, 128.2, 128.7, 128.9 (overlapped), 129.1, 133.4, 136.2, 138.9, $139.9,142.2,196.5$.

For syn isomer. ${ }^{1} \mathrm{H}-\mathrm{NMR}\left(270 \mathrm{MHz}, \mathrm{CDCl}_{3}\right) \delta 3.33(\mathrm{dd}, J=16.8,2.9 \mathrm{~Hz}, 1 \mathrm{H}), 3.69(\mathrm{dd}, J=$ 17.1, 7.2 Hz, 1H), 4.34 (ddd, $J=16.8,10.3,3.2 \mathrm{~Hz}, 1 \mathrm{H}), 5.48$ (s, 1H), 5.78 (s, 1H), 5.86 (d, $J=$ $10.6 \mathrm{~Hz}, 1 \mathrm{H}), 6.01(\mathrm{~s}, 1 \mathrm{H}), 7.12-7.57(\mathrm{~m}, 13 \mathrm{H}), 7.66-7.69(\mathrm{~m}, 2 \mathrm{H}) ;{ }^{13} \mathrm{C}-\mathrm{NMR}(67.5 \mathrm{MHz}$, 
$\left.\mathrm{CDCl}_{3}\right) \delta 41.6,44.8,93.6,119.5,126.5,127.5,128.1,128.2,128.3,128.5,128.6,128.7,128.8$, $133.3,136.9,137.5,139.9,142.5,196.6$.

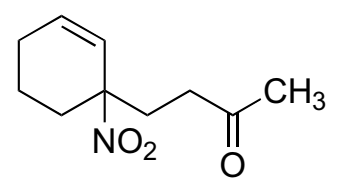

4-((S)-1-nitrocyclohex-2-enyl)-butan-2-one (5e): 5e was purified by flash chromatography (Hexane-EtOAc, v/v 3/1) as yellow oil, isolated yield: 93\%. ${ }^{1} \mathrm{H}-\mathrm{NMR}\left(270 \mathrm{MHz}, \mathrm{CDCl}_{3}\right) \delta 1.40$ $1.56(\mathrm{~m}, 1 \mathrm{H}), 1.59-1.73(\mathrm{~m}, 2 \mathrm{H}), 1.86-2.16(\mathrm{~m}, 7 \mathrm{H}), 2.36-2.52(\mathrm{~m}, 3 \mathrm{H}), 5.81$ (ddd, $J=10.1,3.6$, $2.1 \mathrm{~Hz} 1 \mathrm{H}), 6.02(\mathrm{ddd}, J=10.1,4.1,3.5 \mathrm{~Hz}, 1 \mathrm{H}) ;{ }^{13} \mathrm{C}-\mathrm{NMR}\left(67.5 \mathrm{MHz}, \mathrm{CDCl}_{3}\right) \delta 18.8,24.8$, 30.0, 31.9, 34.0, 37.6, 88.4, 125.1, 134.2, 206.4; IR (cm¹) : 2294, 2331, 1708, 1534, 1444; HRMS Calculated for $\left[\mathrm{C}_{10} \mathrm{H}_{15} \mathrm{NO}_{3} \mathrm{Na}\right]^{+}: 220.0950$, Found: 220.0945.

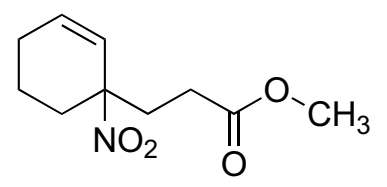

Methyl-3-((s)-1-nitrocyclohex-2-enyl) propionate (5f): $5 f$ was purified by flash chromatography (Hexane-EtOAc, v/v 3/1) as yellow oil, isolated yield: 85\%. ${ }^{1} \mathrm{H}-\mathrm{NMR}(270$ $\left.\mathrm{MHz}, \mathrm{CDCl}_{3}\right) \delta 1.40-1.75(\mathrm{~m}, 3 \mathrm{H}), 1.85-2.33(\mathrm{~m}, 6 \mathrm{H}), 2.38-2.54(\mathrm{~m}, 1 \mathrm{H}), 3.60(\mathrm{~s}, 3 \mathrm{H}), 5.81$ (ddd, $J=9.9,3.7,2.2 \mathrm{~Hz}, 1 \mathrm{H}), 6.02$ (ddd, $J=10.2,4.2,3.2 \mathrm{~Hz}, 1 \mathrm{H}) ;{ }^{13} \mathrm{C}-\mathrm{NMR}(67.5 \mathrm{MHz}$, $\left.\mathrm{CDCl}_{3}\right) \delta 18.8,24.7,28.5,31.6,35.3,51.9,88.3,124.9,134.4,172.5$; IR (cm-1) : 2956, 2331, 1742, 1538, 1448; HRMS Calculated for $\left[\mathrm{C}_{10} \mathrm{H}_{15} \mathrm{NO}_{4} \mathrm{Na}\right]^{+}: 236.0899$, Found: 236.0896.<smiles>CC(=O)CC(C)C1([N+](=O)[O-])C=CCCC1</smiles>

4-Methyl-5-((S)-1-nitrocyclohex-2-enyl) pentan-2-one $(\mathrm{d} / \mathrm{r}=1.6 / 1)(5 \mathrm{~g}): 5 \mathrm{~g}$ was purified by flash chromatography (Hexane-EtOAc, v/v 3/1) as yellow oil, isolated yield: $81 \%$. The two diastereomers cannot be separated. Two sets of signals were obtained for the ${ }^{1} \mathrm{H}$ and ${ }^{13} \mathrm{C}$ NMR. ${ }^{1} \mathrm{H}$ NMR $\left(270 \mathrm{MHz}, \mathrm{CDCl}_{3}\right) \delta 0.77(\mathrm{~d}, J=6.6 \mathrm{~Hz}, 3 \mathrm{H}), 0.81(\mathrm{~d}, \mathrm{~J}=6.6 \mathrm{~Hz}, 3 \mathrm{H}), 1.28-2.53(\mathrm{~m}$, $16 \mathrm{H}), 2.04(\mathrm{~s}, 3 \mathrm{H}), 2.05(\mathrm{~s}, 3 \mathrm{H}), 2.65-2.74(\mathrm{~m}, 2 \mathrm{H}), 5.71-5.79(\mathrm{~m}, 2 \mathrm{H}), 5.99-6.08(\mathrm{~m}, 2 \mathrm{H})$; Isomer 1: ${ }^{13} \mathrm{C} \mathrm{NMR}\left(67.5 \mathrm{MHz}, \mathrm{CDCl}_{3}\right) \delta 14.6,18.6,24.9,27.3,30.5,37.2,45.6,92.2,125.4$, 
135.6, 206.1; Isomer 2: ${ }^{13} \mathrm{C}$ NMR (67.5 MHz, $\left.\mathrm{CDCl}_{3}\right) \delta 15.1,18.7,24.8,27.6,30.5,37.1,45.1$, 92.2, 124.9, 135.0, 206.2; HRMS Calculated for $\left[\mathrm{C}_{11} \mathrm{H}_{17} \mathrm{NO}_{3} \mathrm{Na}\right]^{+}: 234.1106$, Found: 214.1101.<smiles>O=C1CCCC(C2([N+](=O)[O-])C=CCCC2)C1</smiles>

$(\boldsymbol{S})-3-((S)-1-n i t r o c y c l o h e x-2-e n y l)$ cyclohexanone $(\mathrm{d}: \mathrm{r}=1.3: 1)(\mathbf{5 h}):$ Both isomers of $\mathbf{5 h}$ were purified by flash chromatography (Hexane-EtOAc, v/v 3/1) as yellow oils, with overall isolated yield: $76 \%$. The two diastereomers cannot be separated. Two sets of signals were obtained for the ${ }^{1} \mathrm{H}$ and ${ }^{13} \mathrm{C}$ NMR. ${ }^{1} \mathrm{H}-\mathrm{NMR}\left(270 \mathrm{MHz}, \mathrm{CDCl}_{3}\right) \delta$ 1.31-2.62 (m, 30H), 5.81-5.92 (m, 2H), 6.08-6.19 (m, 2H); Isomer $1:{ }^{13} \mathrm{C}-\mathrm{NMR}\left(67.5 \mathrm{MHz}, \mathrm{CDCl}_{3}\right) \delta 18.8,24.5,25.0,25.9,28.5,41.0$, 42.5, 46.5, 91.8, 123.7, 135.8, 209.2; Isomer 2: ${ }^{13} \mathrm{C}$ - NMR (67.5 MHz, $\left.\mathrm{CDCl}_{3}\right) \delta$ 18.7, 24.5, 24.8, 25.6, 27.4, 41.0, 42.7, 46.7, 91.6, 124.7, 135.4, 209.2; HRMS Calculated for $\left[\mathrm{C}_{12} \mathrm{H}_{17} \mathrm{NO}_{3} \mathrm{Na}\right]^{+}$: 246.1106, Found: 246.1101.<smiles>O=C1CCCC(C(C2=CCCCC2)[N+](=O)[O-])C1</smiles>

$(\boldsymbol{S})-3-((\boldsymbol{R})$-cyclohexenylnitromethyl) cyclohexanone $(\mathrm{d}: \mathrm{r}=1.2: 1)(\mathbf{5 i}): \mathbf{5 i}$ was purified by flash chromatography (Hexane-EtOAc, v/v 3/1) as yellow oil, isolated yield: 90\%. The two diastereomers cannot be separated. Two sets of signals were obtained for the ${ }^{1} \mathrm{H}$ and ${ }^{13} \mathrm{C}$ NMR. ${ }^{1} \mathrm{H}-\mathrm{NMR}\left(270 \mathrm{MHz}, \mathrm{CDCl}_{3}\right.$ ) $\delta$ 1.11-1.79 (m, 14H), 1.79-2.07 (m, 12H), 2.10-2.35 (m, 6H), 2.49$2.68(\mathrm{~m}, 2 \mathrm{H}), 4.58(\mathrm{~d}, J=10.6 \mathrm{~Hz}, 1 \mathrm{H}), 4.60(\mathrm{~d}, J=11.8 \mathrm{~Hz}, 1 \mathrm{H}), 5.87-5.90(\mathrm{br}, 2 \mathrm{H})$; Isomer 1: ${ }^{13} \mathrm{C}-\mathrm{NMR}\left(67.5 \mathrm{MHz}, \mathrm{CDCl}_{3}\right) \delta$ 21.7, 22.3, 23.7, 24.3, 25.5, 26.9, 38.4, 41.0, 44.4, 98.9, 130.7, 133.3, 208.5; Isomer 2: ${ }^{13} \mathrm{C}-\mathrm{NMR}\left(67.5 \mathrm{MHz}, \mathrm{CDCl}_{3}\right) \delta$ 21.6, 22.2, 23.6, 24.2, 25.5, 28.2, 38.3, 41.0, 43.1, 98.8, 130.2, 133.7, 209.1; IR ( $\left.\mathrm{cm}^{-1}\right)$ : 2294, 2331, 1713, 1534, 1439; HRMS Calculated for $\left[\mathrm{C}_{13} \mathrm{H}_{19} \mathrm{NO}_{3} \mathrm{Na}\right]^{+}: 260.1263$, Found: 260.1258 . 


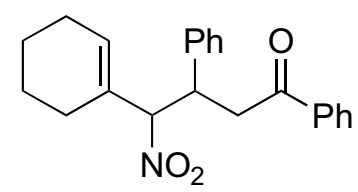

4-cyclohexenyl-4-nitro-1,3-diphenylbutan-1-one $(\mathbf{5 j}) ; \mathbf{5 j}$ was purified by flash chromatography (Hexane-EtOAc, v/v 3/1) with overall isolated yield: 70\% $(\mathrm{d}: \mathrm{r}=4: 1)$. The two diastereomers were separated by column chromatography. Isomer 1 (major) is white solid, m.p. is $186{ }^{\circ} \mathrm{C}$; isomer 2 (minor) colorless oil.

Isomer 1: ${ }^{1} \mathrm{H}-\mathrm{NMR}\left(270 \mathrm{MHz}, \mathrm{CDCl}_{3}\right) \delta 1.24-1.43(\mathrm{~m}, 4 \mathrm{H}), 1.71-2.04(\mathrm{~m}, 4 \mathrm{H}), 3.19(\mathrm{dd}$, $\mathrm{J}=16.6 \mathrm{~Hz}, 1 \mathrm{H}), 3.49(\mathrm{dd}, \mathrm{J}=16.8 \mathrm{~Hz}, 1 \mathrm{H}), 4.2-4.32(\mathrm{~m}, 1 \mathrm{H}), 5.21(\mathrm{~d}, \mathrm{~J}=11.1 \mathrm{~Hz}, 1 \mathrm{H}), 5.77(\mathrm{~s}, 1 \mathrm{H})$, 7.12-7.24(m,1H), 7.36-7.41(m, 2H), 7.47-7.53(m,1H), 7.79-7.82(m, 2H); ${ }^{13} \mathrm{C}-\mathrm{NMR}(67.5 \mathrm{MHz}$, $\left.\mathrm{CDCl}_{3}\right) \delta 21.53,22.2,24.5,42.1,97.8,127.4,128.0,132.9,133.3,196.7$; IR (cm-1) : 2274, 2355, 1709, 1534, 1469; HRMS Calculated for $\left[\mathrm{C}_{22} \mathrm{H}_{23} \mathrm{NO}_{3} \mathrm{Na}\right]^{+}: 372.1575$, Found: 372.15732; Isomer 2: ${ }^{1} \mathrm{H}-\mathrm{NMR}\left(270 \mathrm{MHz}, \mathrm{CDCl}_{3}\right) \delta 1.50-1.63(\mathrm{~m}, 4 \mathrm{H}), 1.99-2.18(\mathrm{~m}, 4 \mathrm{H}), 3.13(\mathrm{dd}, \mathrm{J}=4.2 \mathrm{~Hz}, 1 \mathrm{H})$, 3.23(dd, J=8.9Hz, 1H), 4.34-4.38(m 1H), 5.30(d, J=11.8Hz, 1H), 6.13(s, 1H), 7.21-7.42(m, 7H); ${ }^{13} \mathrm{C}-\mathrm{NMR}\left(67.5 \mathrm{MHz}, \mathrm{CDCl}_{3}\right) \delta 21.7,22.3,23.7,25.6,41.2,98.8,127.6,128.0,133.3,134.4$, 196.7<smiles>CC(=O)CC(C)C(C1=CCCCC1)[N+](=O)[O-]</smiles>

(4R, 5R)-5-methyl-5-nitropentan-2-one (5k): Both isomers of $5 \mathbf{k}$ was purified by flash chromatography (Hexane-EtOAc, v/v 3/1) as yellow oils with overall isolated yield: $81 \%(\mathrm{~d} / \mathrm{r}=$ 1.6/1). The two diastereomers were separated by column chromatography.

Isomer-1 (major): ${ }^{1} \mathrm{H}-\mathrm{NMR}\left(270 \mathrm{MHz}, \mathrm{CDCl}_{3}\right) \delta 0.89(\mathrm{~d}, J=6.9 \mathrm{~Hz}, 3 \mathrm{H}), 1.52-1.69(\mathrm{~m}, 5 \mathrm{H})$, 2.01-2.19 (m, 6H), $2.33(\mathrm{dd}, J=17.1,8.9 \mathrm{~Hz}, 1 \mathrm{H}), 2.45$ (dd, $J=17.1,3.2 \mathrm{~Hz}, 1 \mathrm{H}), 2.80-2.90$ $(\mathrm{m}, 1 \mathrm{H}), 4.69(\mathrm{~d}, J=10.9 \mathrm{~Hz}, 1 \mathrm{H}), 5.90-6.00(\mathrm{br}, 1 \mathrm{H}) ;{ }^{13} \mathrm{C}-\mathrm{NMR}\left(67.5 \mathrm{MHz}, \mathrm{CDCl}_{3}\right) \delta 15.90$, 21.8, 22.3, 23.7, 25.5, 29.8, 30.5, 46.6, 98.6, 130.1, 132.7, 206.3; HRMS Calculated for $\left[\mathrm{C}_{12} \mathrm{H}_{19} \mathrm{NO}_{3} \mathrm{Na}\right]^{+}:$248.1263, Found: 248.1258.

Isomer-2 (minor): ${ }^{1} \mathrm{H}-\mathrm{NMR}\left(270 \mathrm{MHz}, \mathrm{CDCl}_{3}\right) \delta 0.98(\mathrm{~d}, J=6.2 \mathrm{~Hz}, 3 \mathrm{H}), 1.45-1.74(\mathrm{~m}, 4 \mathrm{H})$, 1.89-2.20 (m 7H), $2.26(\mathrm{dd}, J=17.6,8.9 \mathrm{~Hz}, 1 \mathrm{H}), 2.41(\mathrm{dd}, J=17.3,3.5 \mathrm{~Hz}, 1 \mathrm{H}), 2.83-3.06(\mathrm{~m}$, 
$1 \mathrm{H}), 4.72(\mathrm{~d}, J=11.1 \mathrm{~Hz}, 1 \mathrm{H}), 5.90-6.10(\mathrm{br}, 1 \mathrm{H}) ;{ }^{13} \mathrm{C}-\mathrm{NMR}\left(67.5 \mathrm{MHz}, \mathrm{CDCl}_{3}\right) \delta 16.9,21.7$, $22.3,23.8,25.5,29.8,30.7,45.6,99.2,131.3,133.2,206.1$.

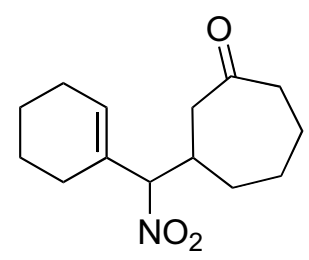

$(\boldsymbol{S})-3-((\boldsymbol{R})$-cyclohexenylnitromethyl-cyclopentanone $(\mathrm{d}: \mathrm{r}=1: 1)(\mathbf{5 l}): \mathbf{5 l}$ was purified by flash chromatography (Hexane-EtOAc, v/v 3/1) as yellow oil, isolated yield: $76 \%$. The two diastereomers cannot be separated. Two sets of signals were obtained for the ${ }^{1} \mathrm{H}$ and ${ }^{13} \mathrm{C}$ NMR. ${ }^{1} \mathrm{H}-\mathrm{NMR}\left(270 \mathrm{MHz}, \mathrm{CDCl}_{3}\right) \delta$ 1.08-2.64 (m, 38H), $4.60(\mathrm{~s}, 1 \mathrm{H}), 4.65(\mathrm{~s}, 1 \mathrm{H}), 5.92-6.08(\mathrm{br}, 2 \mathrm{H})$; Isomer 1 (major): ${ }^{13} \mathrm{C}-\mathrm{NMR}\left(67.5 \mathrm{MHz}, \mathrm{CDCl}_{3}\right) \delta 21.7,22.2,23.5,24.4,25.5,28.2,33.5,36.0$, 43.6, 44.9, 99.2, 130.8, 134.1, 211.8; Isomer 2 (minor): ${ }^{13} \mathrm{C}-\mathrm{NMR}\left(67.5 \mathrm{MHz}, \mathrm{CDCl}_{3}\right) \delta 21.7$, 22.3, 23.8, 24.6, 25.5, 28.5, 31.4, 35.7, 43.6, 46.4, 98.8, 131.0, 133.7, 211.3; HRMS Calculated for $\left[\mathrm{C}_{14} \mathrm{H}_{21} \mathrm{NO}_{3} \mathrm{Na}\right]^{+}: 274.1419$, Found: 274.1414<smiles>CCCCCC(C)(CC(C)=O)C(C)C=O</smiles>

(E)-methyl 3,4-dimethyl-4-nitrodec-5-enoate (5m): $5 \mathrm{~m}$ was purified by flash chromatography (Hexane-EtOAc, v/v 5/1) as yellow oil, isolated yield: $82 \%$. The two diastereomers cannot be separated. Two sets of signals were obtained for the ${ }^{1} \mathrm{H}$ and ${ }^{13} \mathrm{C}$ NMR. ${ }^{1} \mathrm{H}-\mathrm{NMR}(270 \mathrm{MHz}$, $\left.\mathrm{CDCl}_{3}\right) \delta$ 0.84-0.91(m, $\left.12 \mathrm{H}\right), 1.23-1.40(\mathrm{~m}, 8 \mathrm{H}), 1.55(\mathrm{~s}, 3 \mathrm{H}), 1.58(\mathrm{~s}, 3 \mathrm{H}), 2.07-2.47(\mathrm{~m}, 14 \mathrm{H})$, 2.84-3.01(m, 2H), 5.76-7.83(m, 4H). Isomer $1:{ }^{13} \mathrm{C}-\mathrm{NMR}\left(67.5 \mathrm{MHz}, \mathrm{CDCl}_{3}\right) \delta 13.8,14.8,17.5$, 22.2, 30.4, 31.0, 32.1, 37.1, 45.7, 94.4, 128.7, 135.9, 206.0. Isomer $2:{ }^{13} \mathrm{C}-\mathrm{NMR}(67.5 \mathrm{MHz}$, $\left.\mathrm{CDCl}_{3}\right) \delta 13.8,15.3,16.9,22.1,30.2,30.9,32.0,37.0,45.7,94.5,128.8,135.2,206.1$. IR (cm-1): 2930, 2346, 1718, 1537, 1456; HRMS Calculated for $\left[\mathrm{C}_{13} \mathrm{H}_{23} \mathrm{NO}_{3} \mathrm{Na}\right]^{+}:$264.15756, Found: 264.15695 
<smiles>[B]/C=C/C(C)(CCC(=O)OC)[N+](=O)[O-]</smiles>

(R,E)-methyl 4-methyl-4-nitrodec-5-enoate (5n): 5n was purified by flash chromatography (Hexane-ETOAc, v/v 5/1) as yellow oil, isolated yield: 70\%. ${ }^{1} \mathrm{H}$ NMR $\left(270 \mathrm{MHz}, \mathrm{CDCl}_{3}\right) \delta 0.83$ (t, $J=6.9 \mathrm{~Hz}, 3 \mathrm{H}), 1.97-2.06(\mathrm{~m}, 2 \mathrm{H}), 1.59(\mathrm{~s}, 3 \mathrm{H}), 1.98-2.06(\mathrm{~m}, 2 \mathrm{H}), 2.17-2.39(\mathrm{~m}, 4 \mathrm{H}), 3.61$ (s, 3H), 5.65-5.80 (m, 2H); ${ }^{13} \mathrm{C}$ NMR (67.5 MHz, $\left.\mathrm{CDCl}_{3}\right) \delta 13.9,22.2,29.1,30.9,32.0,34.5$, 51.9, 90.2, 128.7, 134.8, 172.6; IR ( $\left.\mathrm{cm}^{-1} 1\right)$ : 2956, 2346, 1738, 1538, 1437; HRMS Calculated for $\left[\mathrm{C}_{12} \mathrm{H}_{21} \mathrm{NO}_{4} \mathrm{Na}\right]^{+}:$266.1368, Found: 266.1365.<smiles>C=C(C)C(C1CCCC(=O)C1)[N+](=O)[O-]</smiles>

(S)-3-((R)-2-methyl-1-nitroallyl) cyclohexanone $(\mathrm{d} / \mathrm{r}=1.1 / 1)(50)$ : 50 was purified by flash chromatography (Hexane-EtOAc, v/v 5/1) as yellow oil, isolated yield: 67\%. The two diastereomers cannot be separated. Two sets of signals were obtained for the ${ }^{1} \mathrm{H}$ and ${ }^{13} \mathrm{C}$ NMR. ${ }^{1} \mathrm{H}-\mathrm{NMR}\left(270 \mathrm{MHz}, \mathrm{CDCl}_{3}\right) \delta$ 1.21-2.47 (m, 22H), 2.55-2.71 (m, 2H), $4.74(\mathrm{~d}, J=10.4 \mathrm{~Hz}, 1 \mathrm{H})$, $4.75(\mathrm{~d}, J=10.8 \mathrm{~Hz}, 1 \mathrm{H}), 5.19-5.21(\mathrm{~m}, 4 \mathrm{H})$; Isomer 1 (major): ${ }^{13} \mathrm{C}-\mathrm{NMR}\left(67.5 \mathrm{MHz}, \mathrm{CDCl}_{3}\right) \delta$ 17.9, 24.3, 26.8, 38.7, 40.9, 44.2, 97.9, 120.9, 137.2, 208.2; Isomer 2: ${ }^{13} \mathrm{C}-\mathrm{NMR}(67.5 \mathrm{MHz}$, $\left.\mathrm{CDCl}_{3}\right) \delta 17.7,24.2,28.2,38.6,41.0,43.0,97.8,121.4,136.7,208.7$; HRMS Calculated for $\left[\mathrm{C}_{10} \mathrm{H}_{15} \mathrm{NO}_{3} \mathrm{Na}\right]^{+}:$220.0949, Found: 220.0946 .<smiles>C=C(c1ccccc1)C(CC(C)=O)C(C)(C)CC(=O)c1ccccc1</smiles>

(R)-4,4-dimethyl-5-nitro-6-phenylhept-6-en-2-one $\quad(5 p): \quad 5 p$ was purified by flash chromatography (Hexane-EtOAc, v/v 5/1) as yellow oil, isolated yield: 45\%. ${ }^{1} \mathrm{H}$ NMR (270 $\left.\mathrm{MHz}, \mathrm{CDCl}_{3}\right) \delta 1.06(\mathrm{~s}, 3 \mathrm{H}), 1.19(\mathrm{~s}, 3 \mathrm{H}), 1.78(\mathrm{~s}, 3 \mathrm{H}), 2.39(\mathrm{q}, \mathrm{J}=18.03 \mathrm{~Hz}, 2 \mathrm{H}), 5.55(\mathrm{~s}, 1 \mathrm{H})$, 5.70(s, 1H), 7.31-7.37(m, 5H); ${ }^{13} \mathrm{C}-\mathrm{NMR}\left(67.5 \mathrm{MHz}, \mathrm{CDCl}_{3}\right) \delta$ 23.9, 24.2, 31.1, 37.9, 51.1, 93.8, 126.9, 128.3, 128.9, 141.2, 206.7; HRMS Calculated for $\left[\mathrm{C}_{15} \mathrm{H}_{19} \mathrm{NO}_{3} \mathrm{Na}\right]^{+}:$284.12626, Found: 284.12587. 
<smiles>COC(=O)CCC(C1=CCCCC1)[N+](=O)[O-]</smiles>

(R)-Methyl-4-cyclohexenyl-4-nitrobutanoate (5q): 5q was purified by flash chromatography (Hexane-EtOAc, v/v 5/1) as yellow oil, isolated yield: 85\%. ${ }^{1} \mathrm{H} \mathrm{NMR}\left(270 \mathrm{MHz}, \mathrm{CDCl}_{3}\right) \delta 1.38$ $1.65(\mathrm{~m}, 4 \mathrm{H}), 1.80-2.18(\mathrm{~m}, 5 \mathrm{H}), 2.26(\mathrm{t}, J=6.9 \mathrm{~Hz}, 2 \mathrm{H}), 2.32-2.45(\mathrm{~m}, 1 \mathrm{H}), 3.61(\mathrm{~s}, 3 \mathrm{H}), 4.84$ $(\mathrm{t}, J=7.4 \mathrm{~Hz}, 1 \mathrm{H}), 5.86-5.89$ (br, $1 \mathrm{H}) .{ }^{13} \mathrm{C} \mathrm{NMR}\left(67.5 \mathrm{MHz}, \mathrm{CDCl}_{3}\right) \delta 21.7,22.2,23.4,25.3$, 25.7, 30.1, 51.9, 92.4, 131.5, 131.6, 172.4; IR (cm¹) : 2916, 2346, 1706, 1478, 1433; HRMS Calculated for $\left[\mathrm{C}_{11} \mathrm{H}_{17} \mathrm{NO}_{4} \mathrm{Na}\right]^{+}: 250.1055$, Found: 250.1051 .
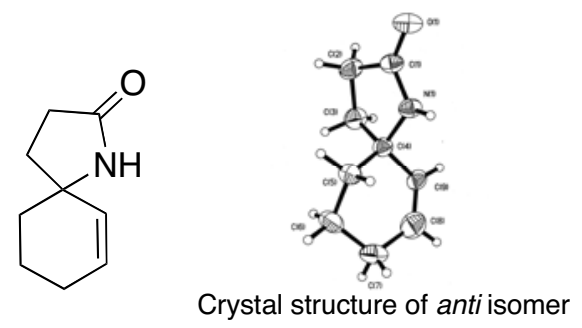

6a: (444 mg, 72\% yield). IR (cm-1) : 3175, 2901, 1975, 1703, 1547, $1425{ }^{1} \mathrm{H}$ NMR (270 MHz, $\left.\mathrm{CDCl}_{3}\right) \delta 1.53-1.69(\mathrm{~m}, 2 \mathrm{H}), 1.85-2.17(\mathrm{~m}, 6 \mathrm{H}), 2.38(\mathrm{dd}, J=8.4,6.9 \mathrm{~Hz}, 2 \mathrm{H}), 5.56(\mathrm{~d}, J=10.2$ $\mathrm{Hz}, 1 \mathrm{H}), 5.93-5.99(\mathrm{~m}, 1 \mathrm{H}) ;{ }^{13} \mathrm{C} \mathrm{NMR}\left(67.5 \mathrm{MHz}, \mathrm{CDCl}_{3}\right) \delta 19.6,24.2,26.3,30.0,30.8,63.3$, 129.8, 132.0, 169.5; HRMS Calculated for $\left[\mathrm{C}_{9} \mathrm{H}_{13} \mathrm{NONa}\right]^{+}:$174.0895, Found: 174.0890.

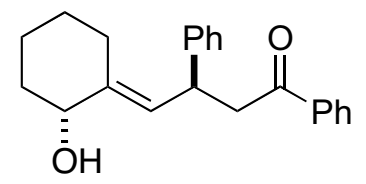

6b: ${ }^{1} \mathrm{H}$ NMR $\left(270 \mathrm{MHz}, \mathrm{CDCl}_{3}\right) \delta$ 1.27-1.48 (m, 4H), 1.60-2.00 (m, 3H), 2.47-2.60 (m, 1H), $3.30(\mathrm{dd}, J=15.8,7.9 \mathrm{~Hz}, 1 \mathrm{H}), 3.39(\mathrm{dd}, J=15.8,6.2 \mathrm{~Hz}, 1 \mathrm{H}), 3.98(\mathrm{dd}, J=6.4,4.1 \mathrm{~Hz}, 1 \mathrm{H})$, $4.36(\mathrm{ddd}, \mathrm{J}=14.4,6.2,3.5 \mathrm{~Hz}, 1 \mathrm{H}), 5.56(\mathrm{~d}, J=9.9 \mathrm{~Hz}, 1 \mathrm{H}), 7.13-7.21(\mathrm{~m}, 1 \mathrm{H}), 7.24-7.29$ (m, $3 \mathrm{H}), 7.36-7.45(\mathrm{~m}, 2 \mathrm{H}), 7.51-7.60(\mathrm{~m}, 1 \mathrm{H}), 7.89-7.93(\mathrm{~m}, 2 \mathrm{H}) ;{ }^{13} \mathrm{C} \mathrm{NMR}\left(67.5 \mathrm{MHz}, \mathrm{CDCl}_{3}\right) \delta$ 23.3, 26.9, 27.1, 36.3, 39.0, 45.9, 73.4, 123.0, 126.4, 127.3, 128.3, 128.6, 128.7, 133.1, 137.2, 142.0, 144.8, 198.9; HRMS Calculated for $\left[\mathrm{C}_{22} \mathrm{H}_{24} \mathrm{NO}_{2} \mathrm{Na}\right]^{+}: 343.1674$, Found: 343.1669. (the absolute stereochemistry of the product is not determined. However, the single diastereomer of $\mathbf{5} \mathbf{j}$ gave single diastereomer of $\mathbf{6 b}$, while the mixture of 1.5:1 syn/anti isomers gave 1.5:1 mixture 
of two stereoisomers. Therefore, the conversion is concerted and the structure of the product can be rationalized by the starting material $\mathbf{5} \mathbf{j}$, whose relative stereo-chemistry was determined by the comparison with the 5d X-ray structure).

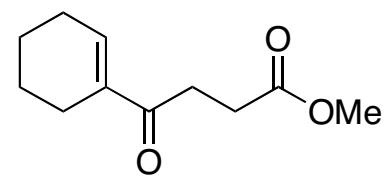

6c: IR (cm-1) : 3394, 2934, 1735, 1666, $1436{ }^{1} \mathrm{H}$ NMR $\left(270 \mathrm{MHz}, \mathrm{CDCl}_{3}\right) \delta$ 1.56-1.67 (m, 4H), 2.21-2.25 (m, 4H), $2.61(\mathrm{t}, J=6.6 \mathrm{~Hz}, 2 \mathrm{H}), 2.97(\mathrm{t}, J=6.6 \mathrm{~Hz}, 2 \mathrm{H}), 3.67(\mathrm{~s}, 3 \mathrm{H}), 6.94-6.97(\mathrm{br}$, $1 \mathrm{H}) ;{ }^{13} \mathrm{C} \mathrm{NMR}\left(67.5 \mathrm{MHz}, \mathrm{CDCl}_{3}\right) \delta 21.5,21.9,23.0,26.0,28.1,31.7,51.7,138.8,140.2,173.7$, 198.9; HRMS Calculated for $\left[\mathrm{C}_{11} \mathrm{H}_{16} \mathrm{NO}_{3} \mathrm{H}\right]^{+}: 197.1178$, Found: 197.1173 . 
IV. The Computation Studies.

The computational studies were performed using the GAMESS-US package at the MP2/6-31G* level of theory. The results are summarized in Scheme S1.

Scheme S1. Computation studies revealed the unfavored equilibrium for simple cabanion addition

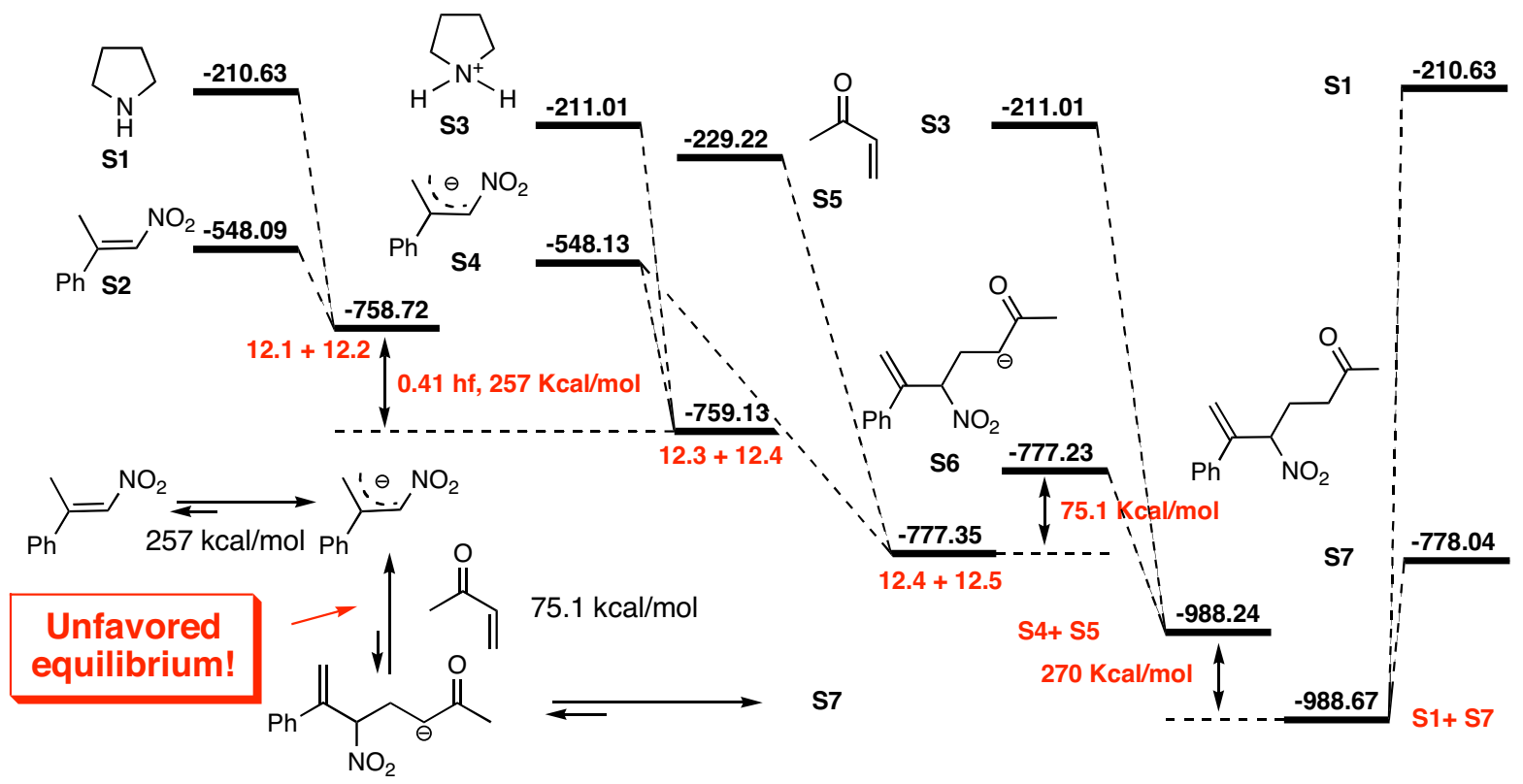

Based on the calculation, a clear unfavored equilibrium for the carbanion $\mathbf{S 4}$ addition to the enone S5 was revealed, which supported the nucleophilic addition mechanism and agreed with the experimental data. 


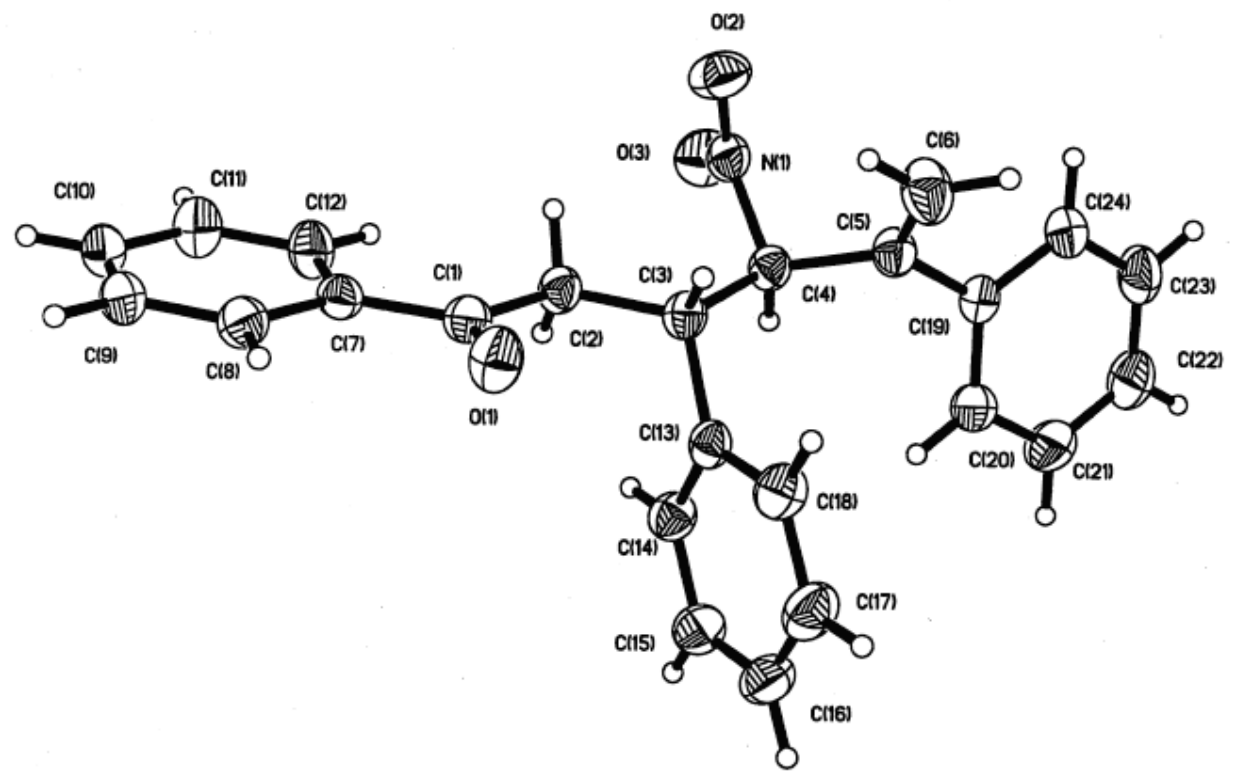

Figure S2. ORTEP drawing of the crystal structure of compound $\mathbf{5 d}$.

Compound 5d: $\mathrm{C}_{24} \mathrm{H}_{21} \mathrm{NO}_{3} ; 371.42 ; 0.22 \times 0.40 \times 0.42 \mathrm{~mm}$; Triclinic; $\mathrm{P} 1 ; \mathrm{a}=5.7208 \mathrm{~A}^{\mathrm{O}}, \mathrm{b}=$ $9.9178 \mathrm{~A}^{\mathrm{O}}, \mathrm{c}=19.0024 \mathrm{~A}^{\mathrm{O},} \alpha=79.479^{\mathrm{O}}, \beta=82.596^{\mathrm{O}}, \gamma=84.090^{\mathrm{O}} ; \mathrm{Vol} .=971.64 \mathrm{~A}^{3} \mathrm{Z}=2 ; \rho=$ $1.270 \mathrm{~g} / \mathrm{cm}^{3} ; \mu=0.84 \mathrm{~cm}^{-1}$; Radiation source: $\operatorname{MoK} \alpha, \lambda=0.71073 \mathrm{~A}^{0} ; \mathrm{T}=293 \mathrm{~K} ; 2 \theta \max =28$; Reflections collected: 7011, Independent reflections 4340; Data collection consisted of the measurement of a total of 1650 frames in five different runs covering a hemisphere of data.; A colorless crystal of $\mathrm{C}_{24} \mathrm{H}_{21} \mathrm{NO}_{3}$ was washed with the perfluoropolyether PFO-XR75 (Lancaster) and wedged in a glass capillary. CCDC \# is 656161. 


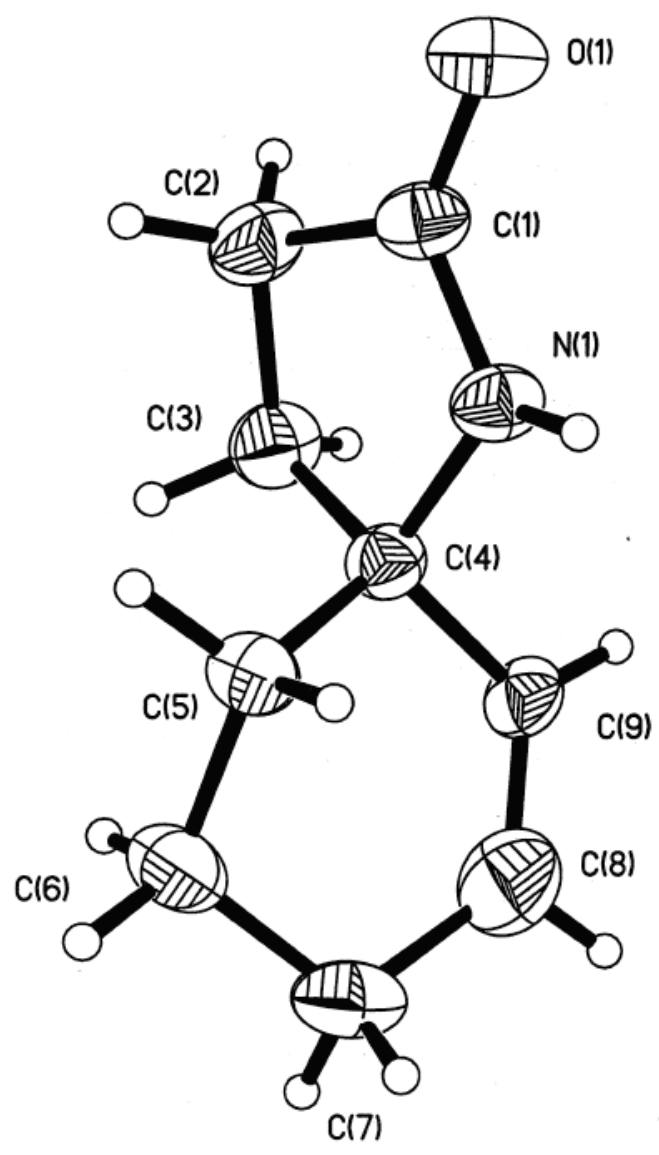

Figure S3. ORTEP drawing of the crystal structure of compound $6 \mathbf{a}$

Compound 6a: $\mathrm{C}_{9} \mathrm{H}_{13} \mathrm{NO} ; 151.20 ; 0.28 \times 0.40 \times 0.42 \mathrm{~mm}$; Monoclinic; $\mathrm{P} 2{ }_{1} / \mathrm{c} ; \mathrm{a}=11.2786 \mathrm{~A}^{\mathrm{O}}, \mathrm{b}$ $=7.629 \mathrm{~A}^{\mathrm{O}}, \mathrm{c}=9.9808 \mathrm{~A}^{\mathrm{O}}, \alpha=90^{\mathrm{O}}, \beta=98.141^{\mathrm{O}}, \gamma=90^{\mathrm{O}} ; \mathrm{Vol} .=850.15 \mathrm{~A}^{3} \mathrm{Z}=4 ; \rho=1.181 \mathrm{~g} / \mathrm{cm}^{3}$; $\mu=0.77 \mathrm{~cm}^{-1}$; Radiation source: $\mathrm{MoK} \alpha, \lambda=0.71073 \mathrm{~A}^{\circ} ; \mathrm{T}=293 \mathrm{~K} ; 2 \theta$ max $=28$; Reflections collected: 5520, Independent reflections 1912; Data collection consisted of the measurement of a total of 1650 frames in five different runs covering a hemisphere of data; A colorless crystal of $\mathrm{C}_{9} \mathrm{H}_{13} \mathrm{NO}$ was washed with the perfluoropolyether PFO-XR75 (Lancaster) and wedged in a glass capillary. CCDC \# is 656162. 
VII. ${ }^{1}$ H-NMR and ${ }^{13}$ C-NMR Spectra

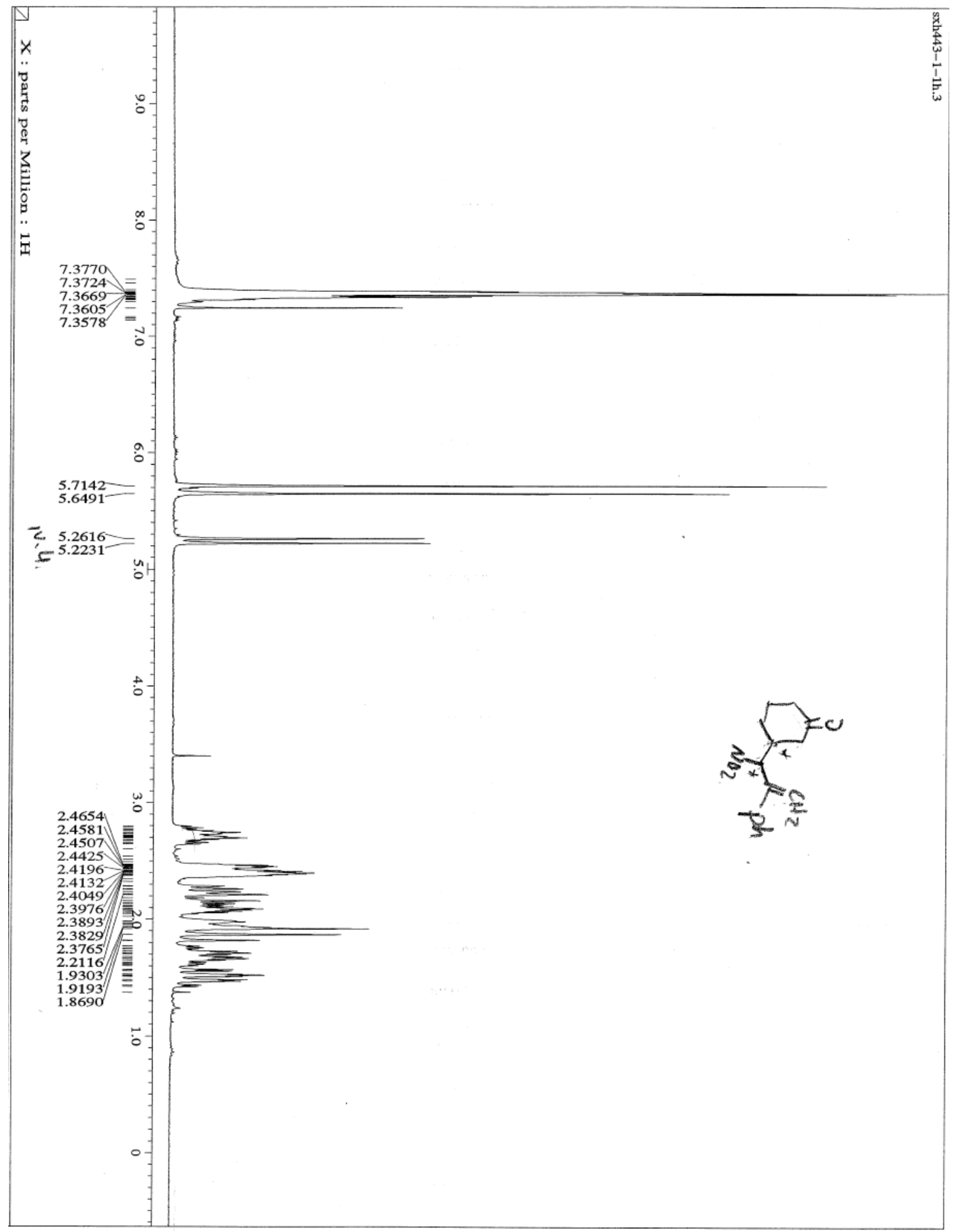




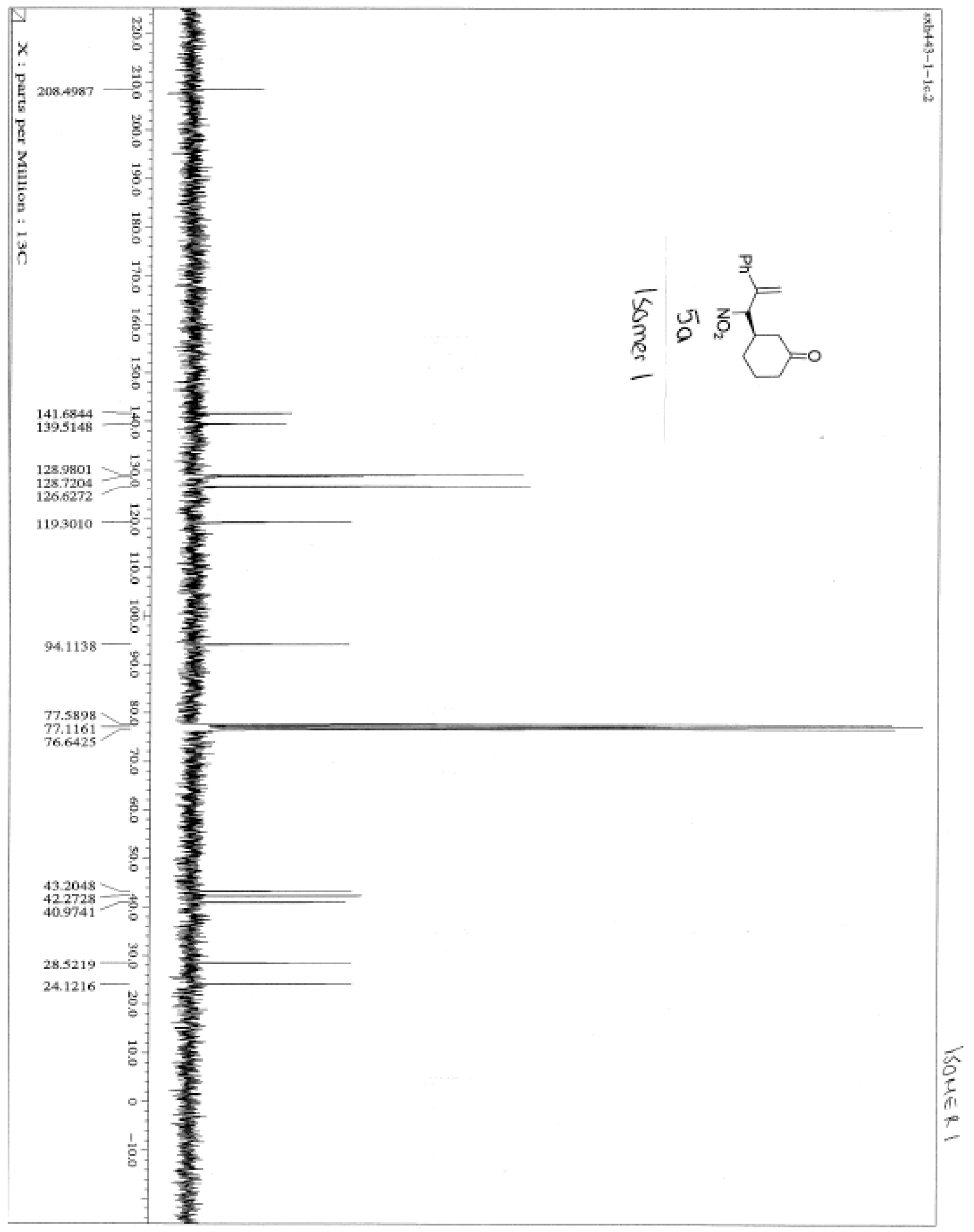




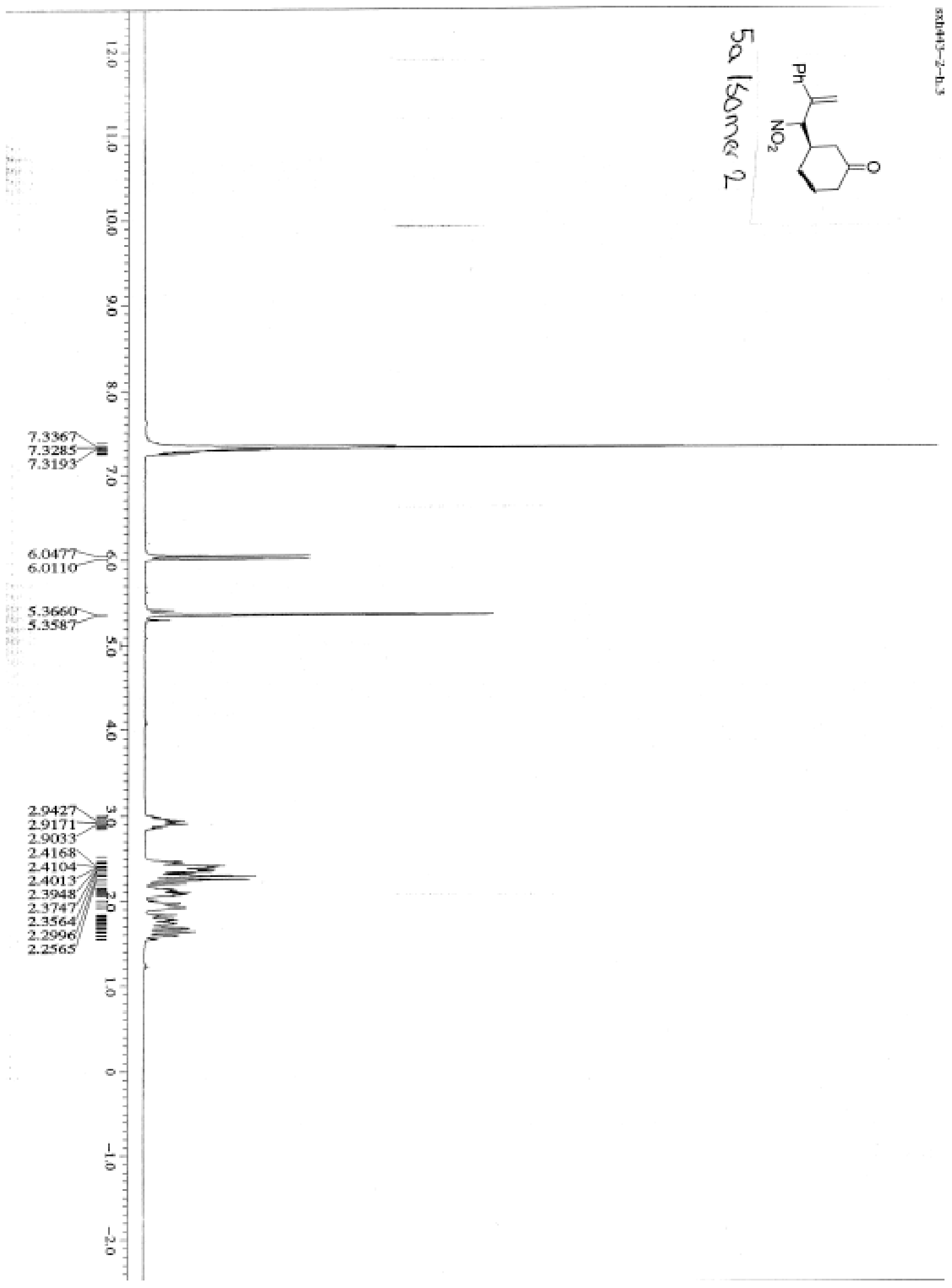

S-19 


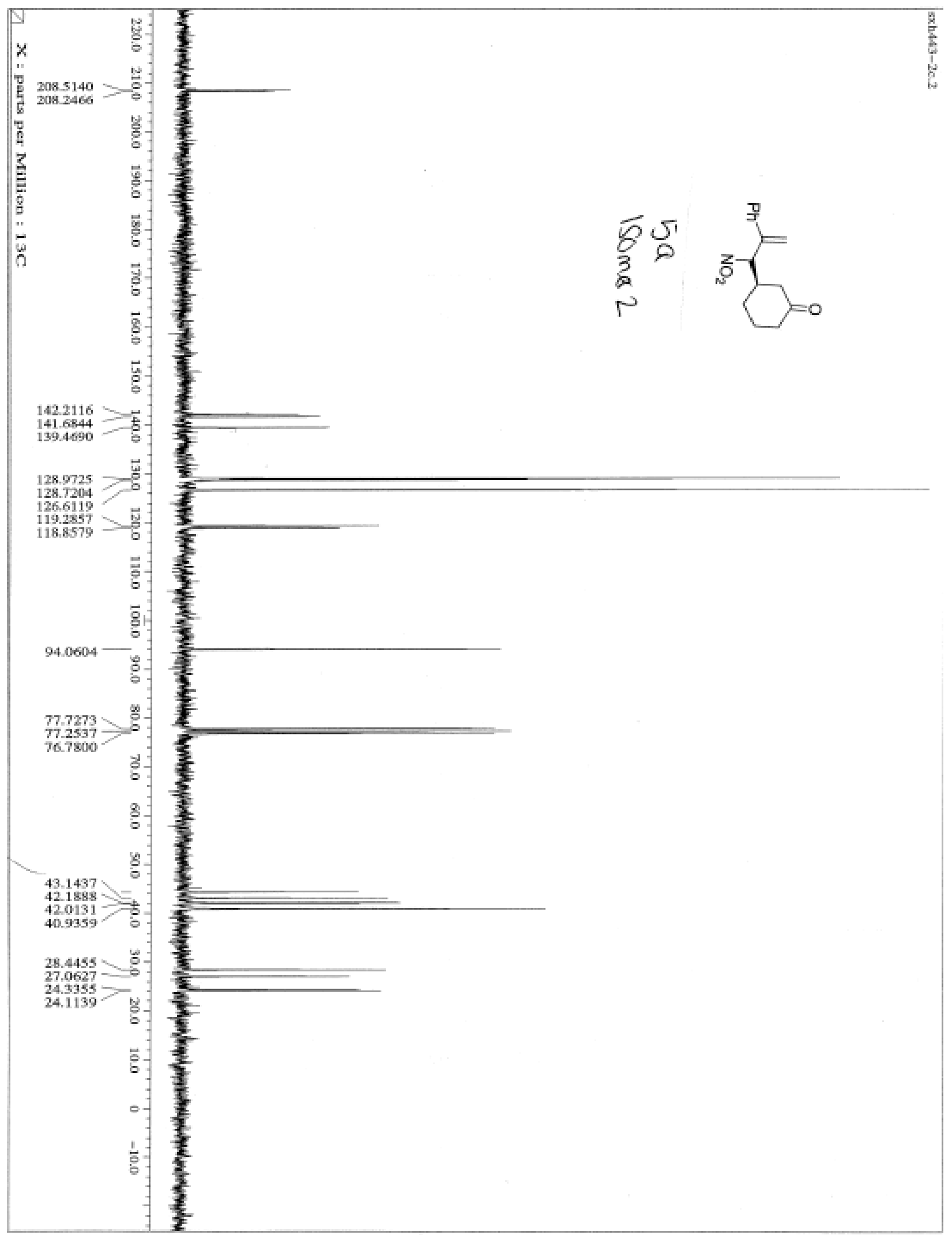




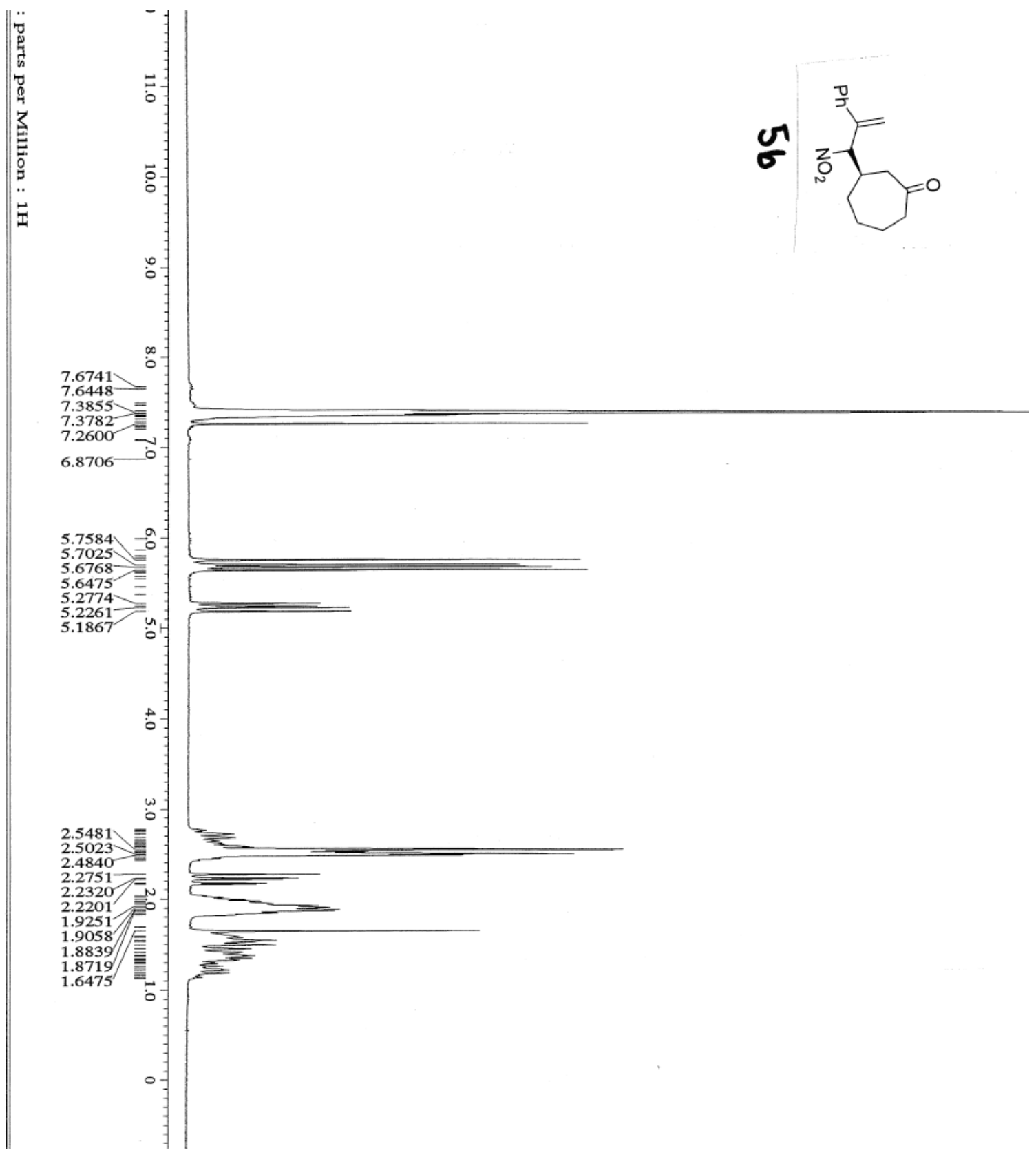




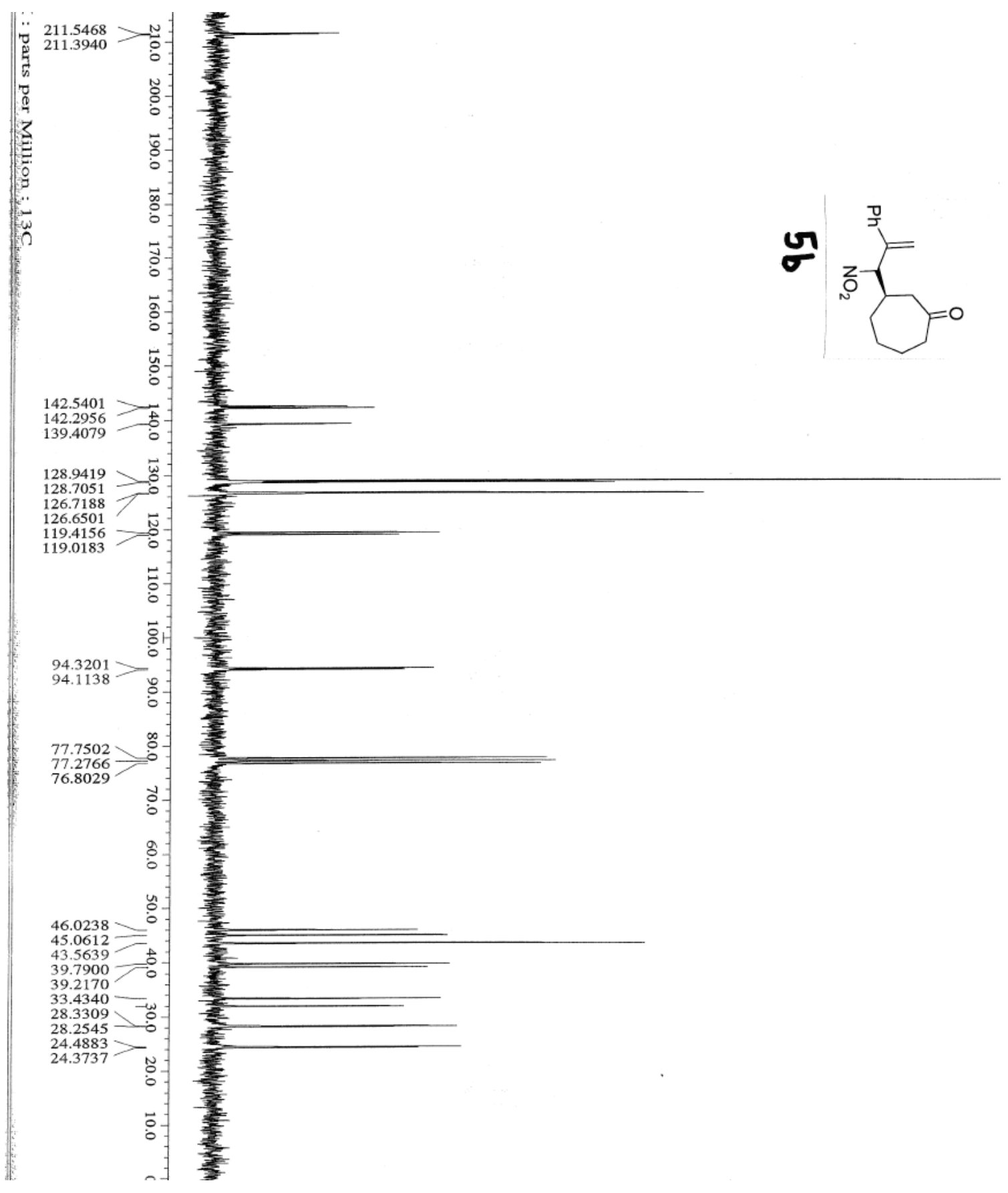




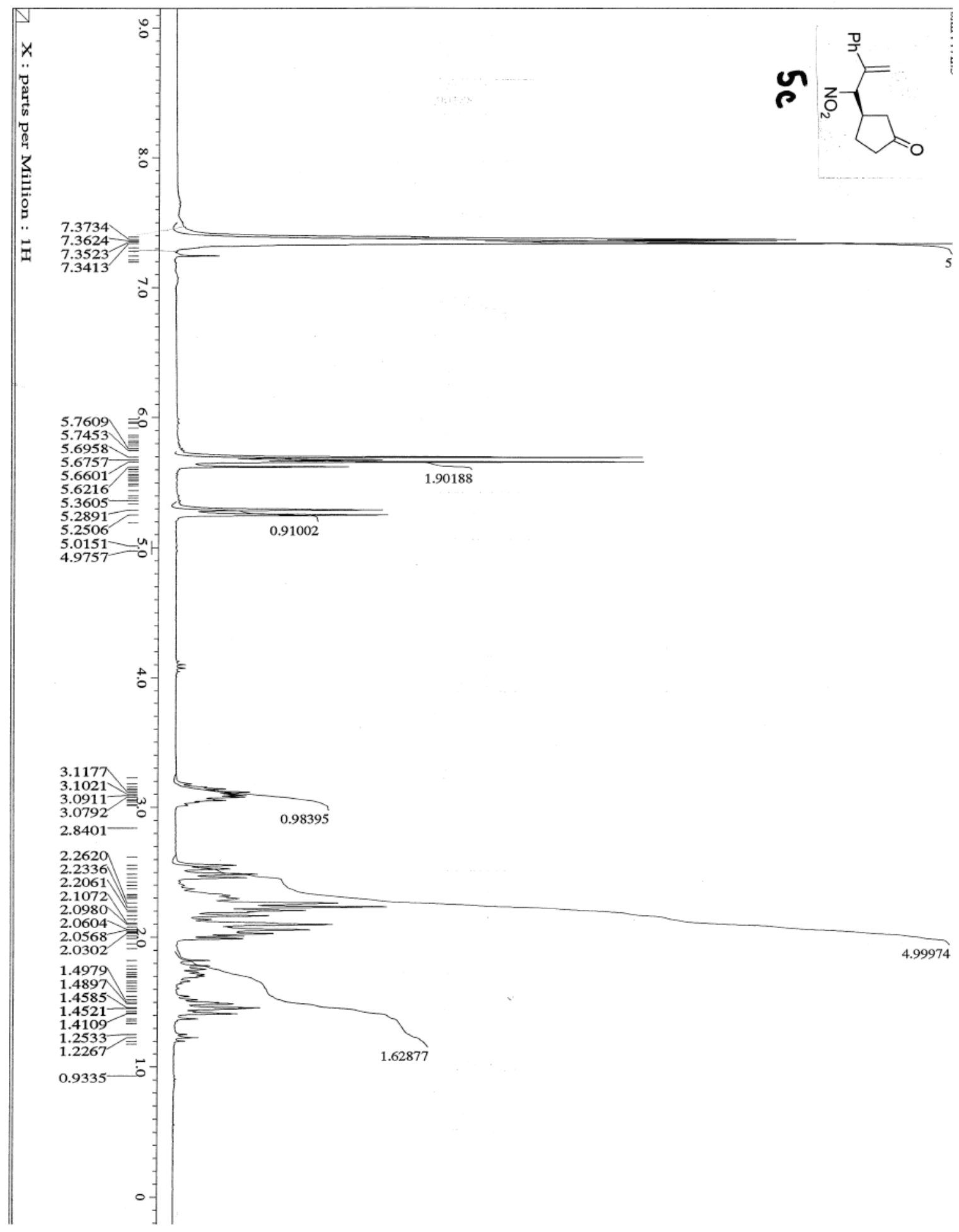

S-23 


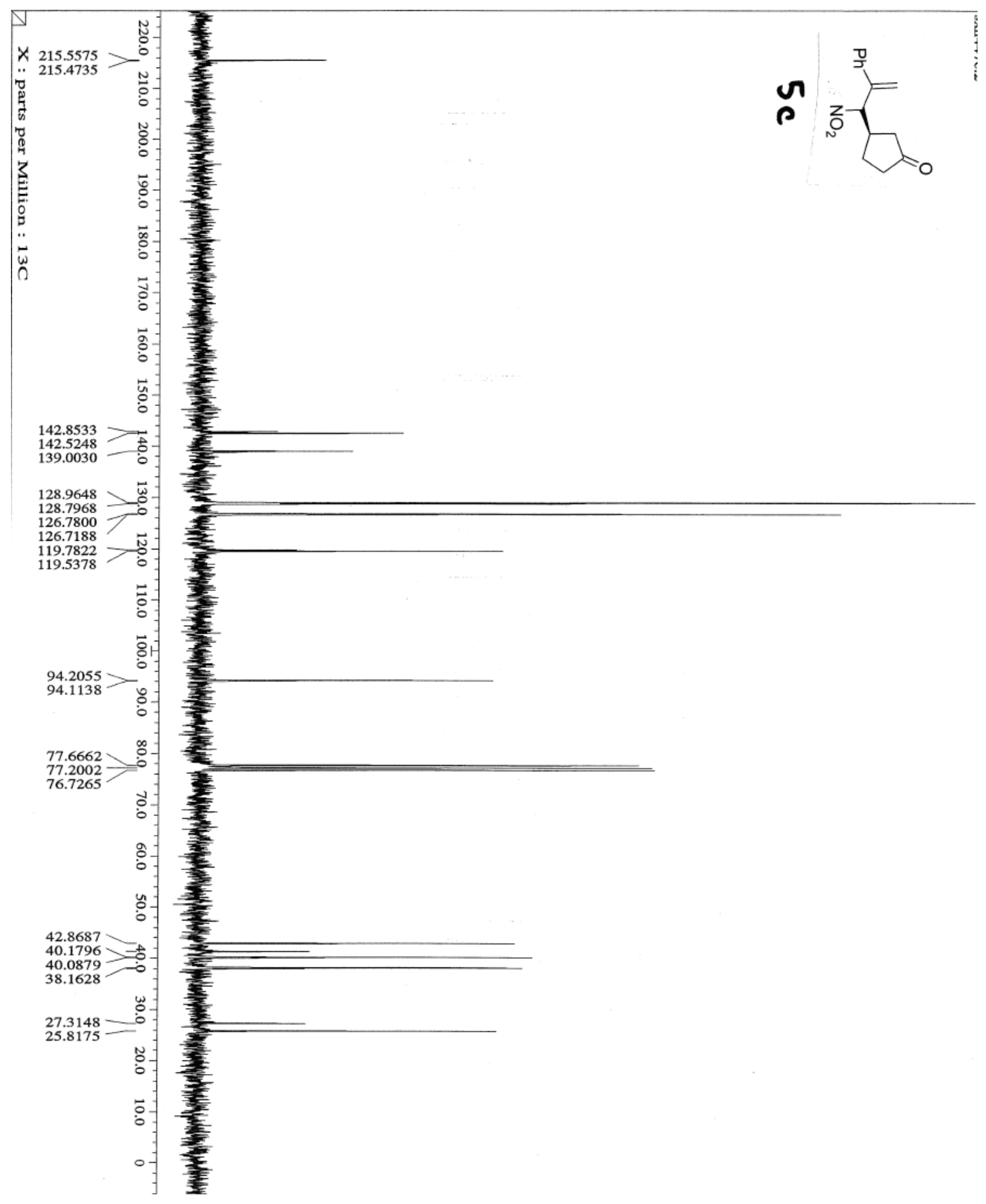




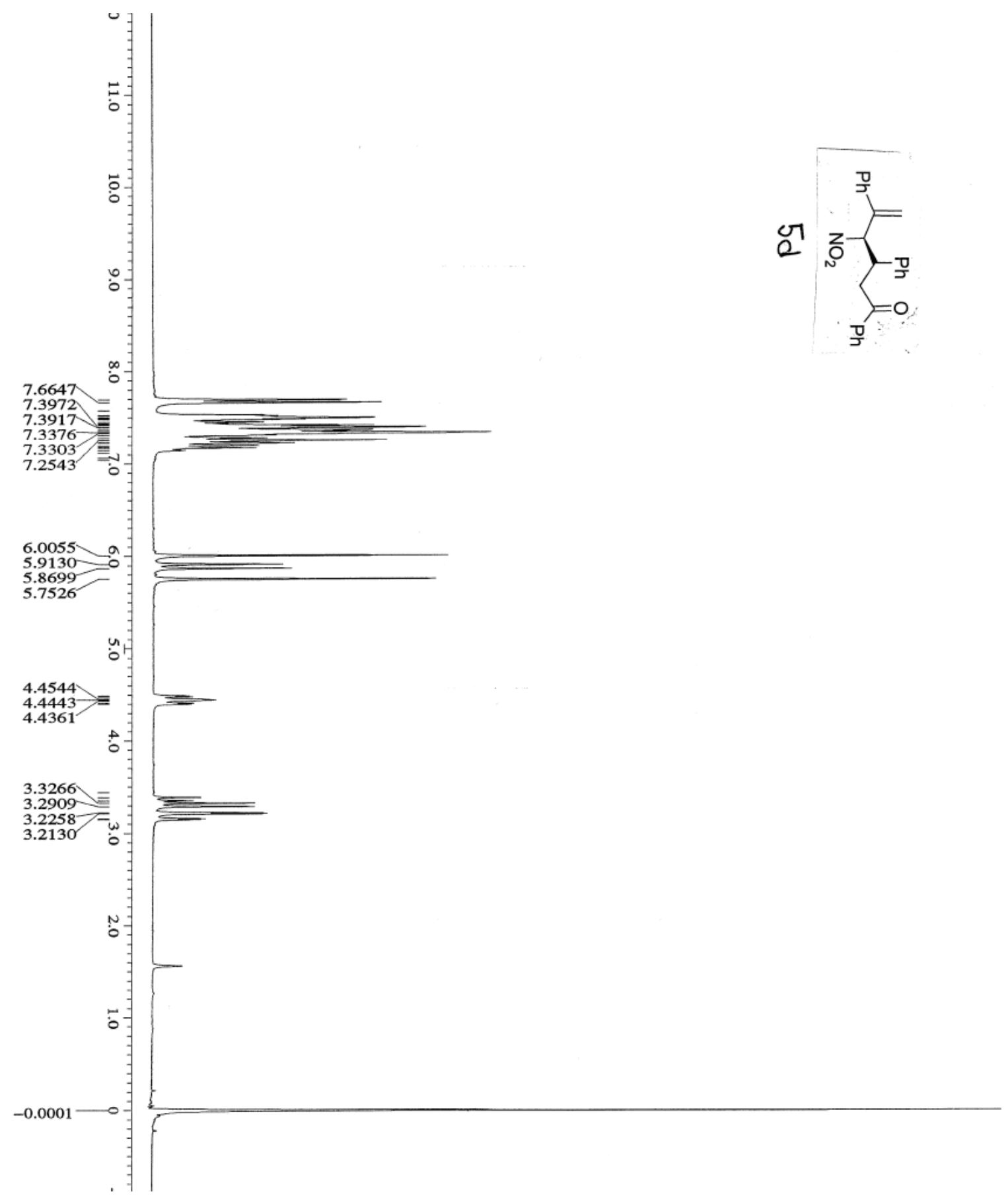

S-25 


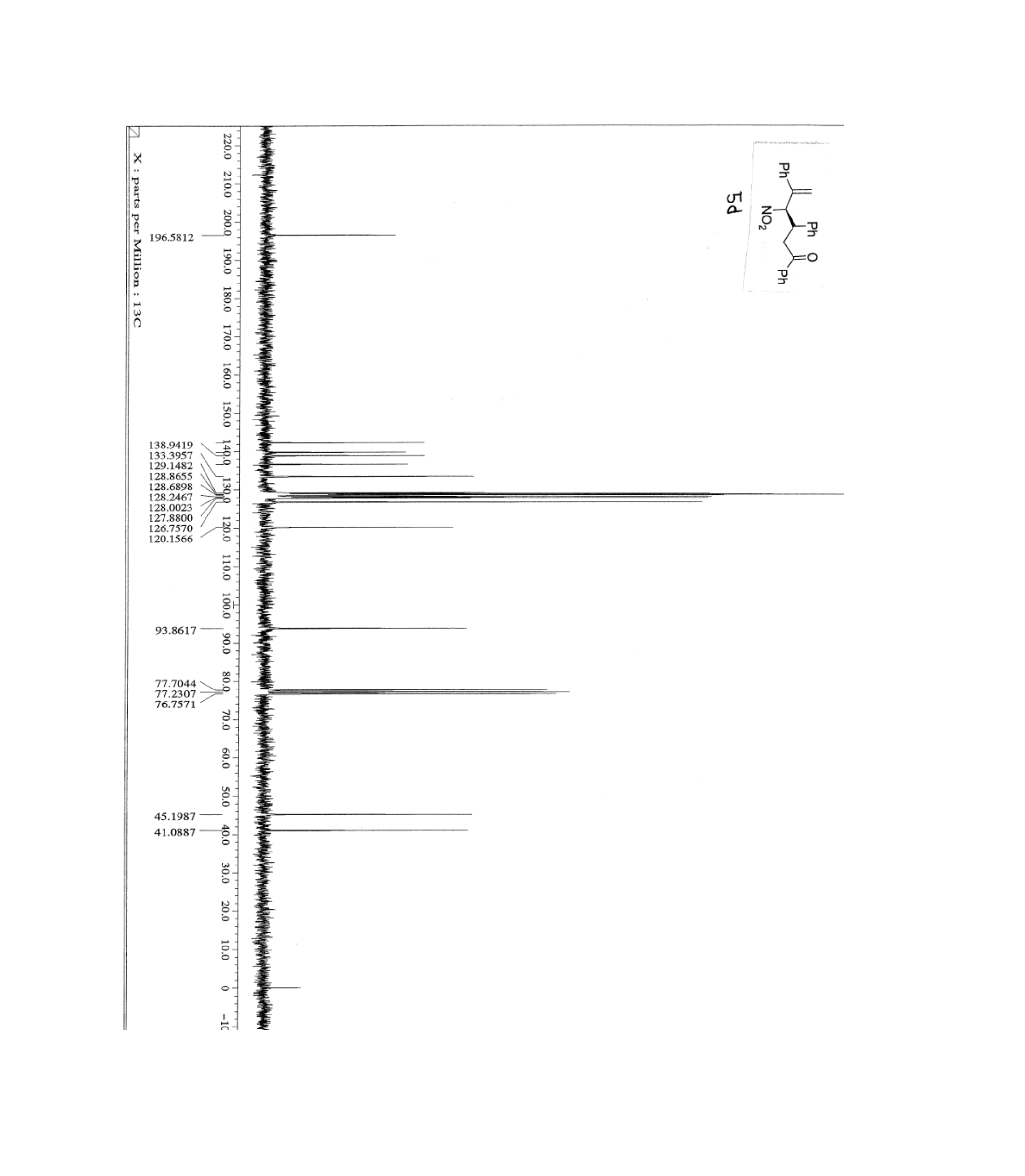




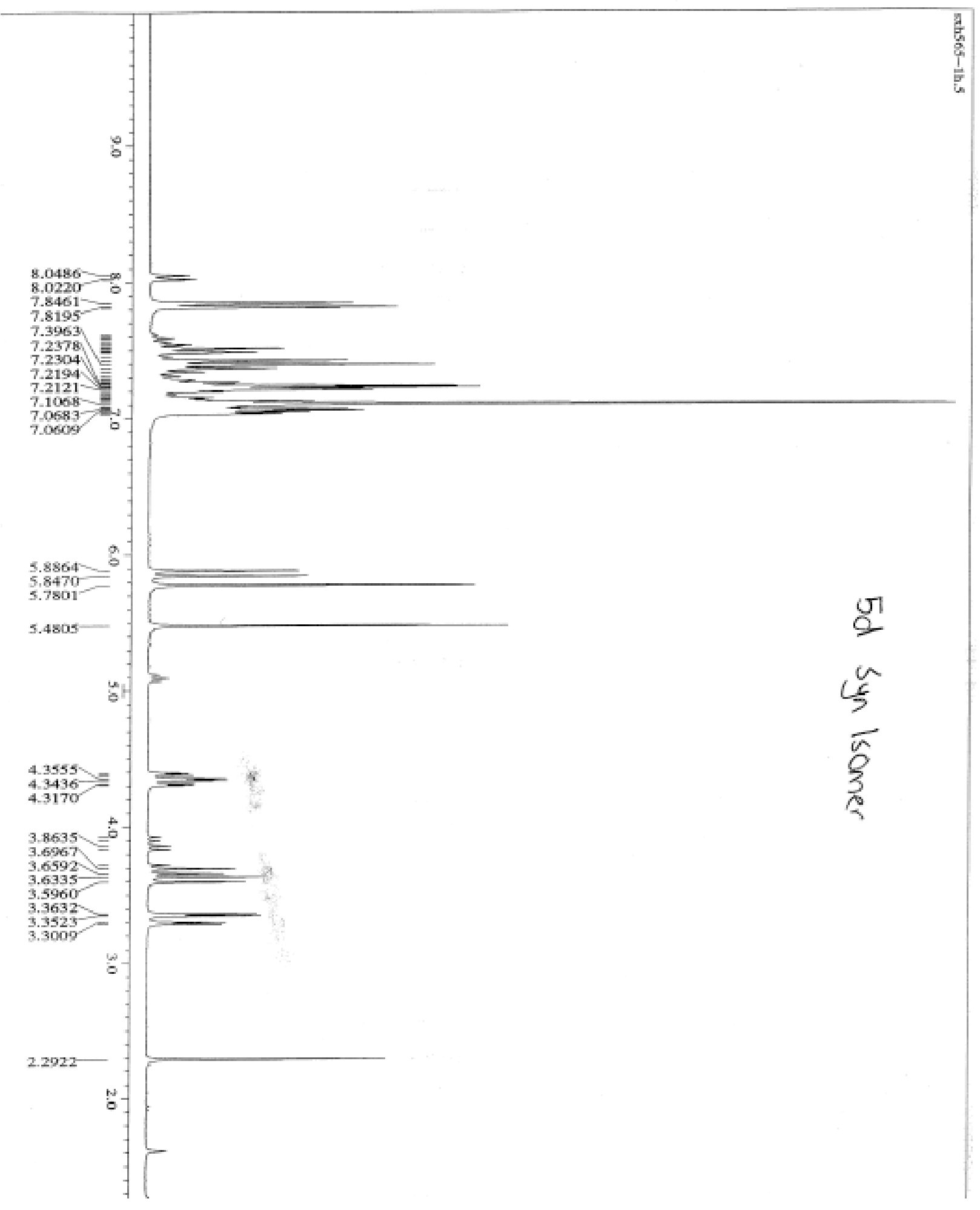




$$
\text { : }
$$




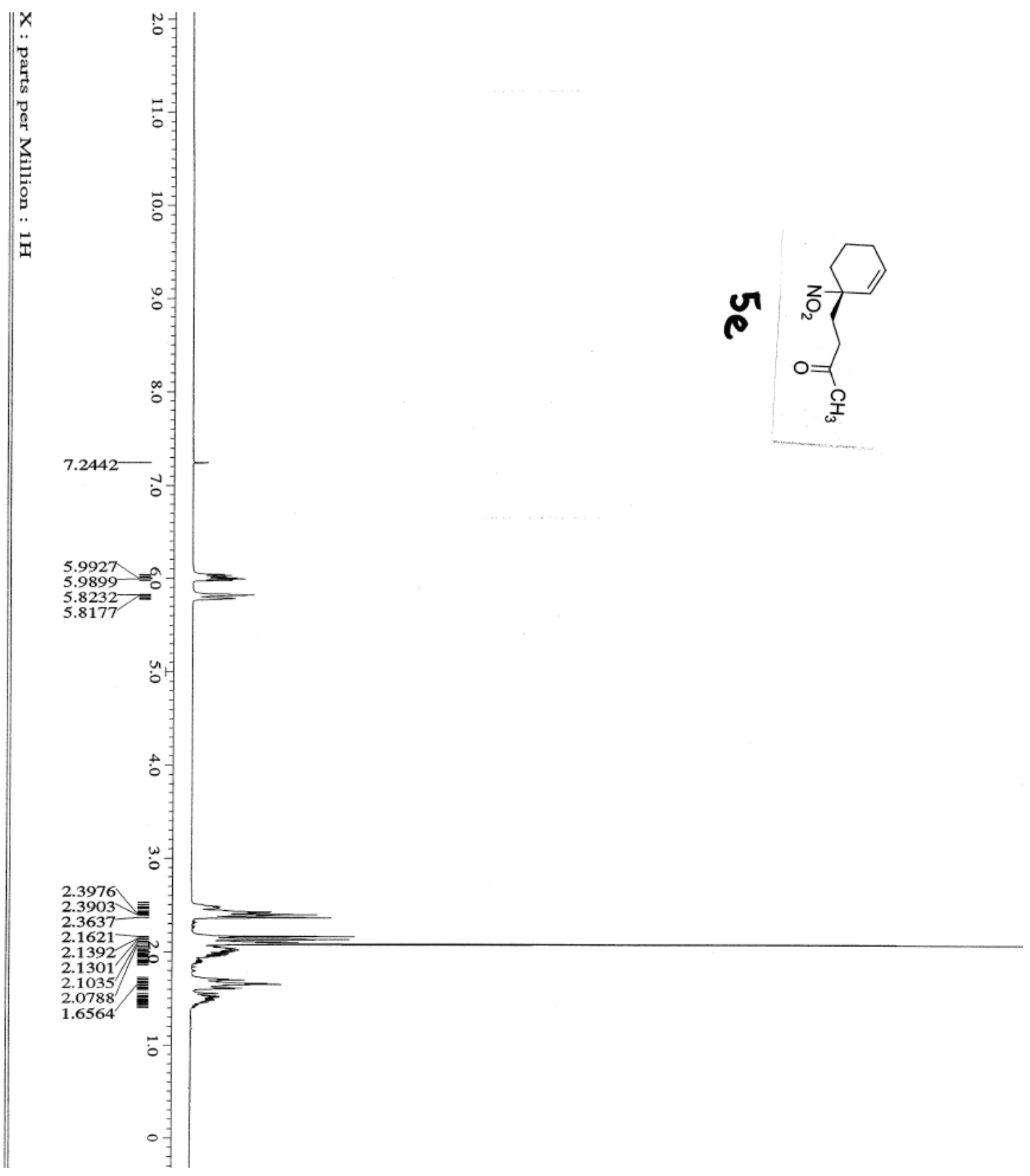




$$
\text { L }
$$




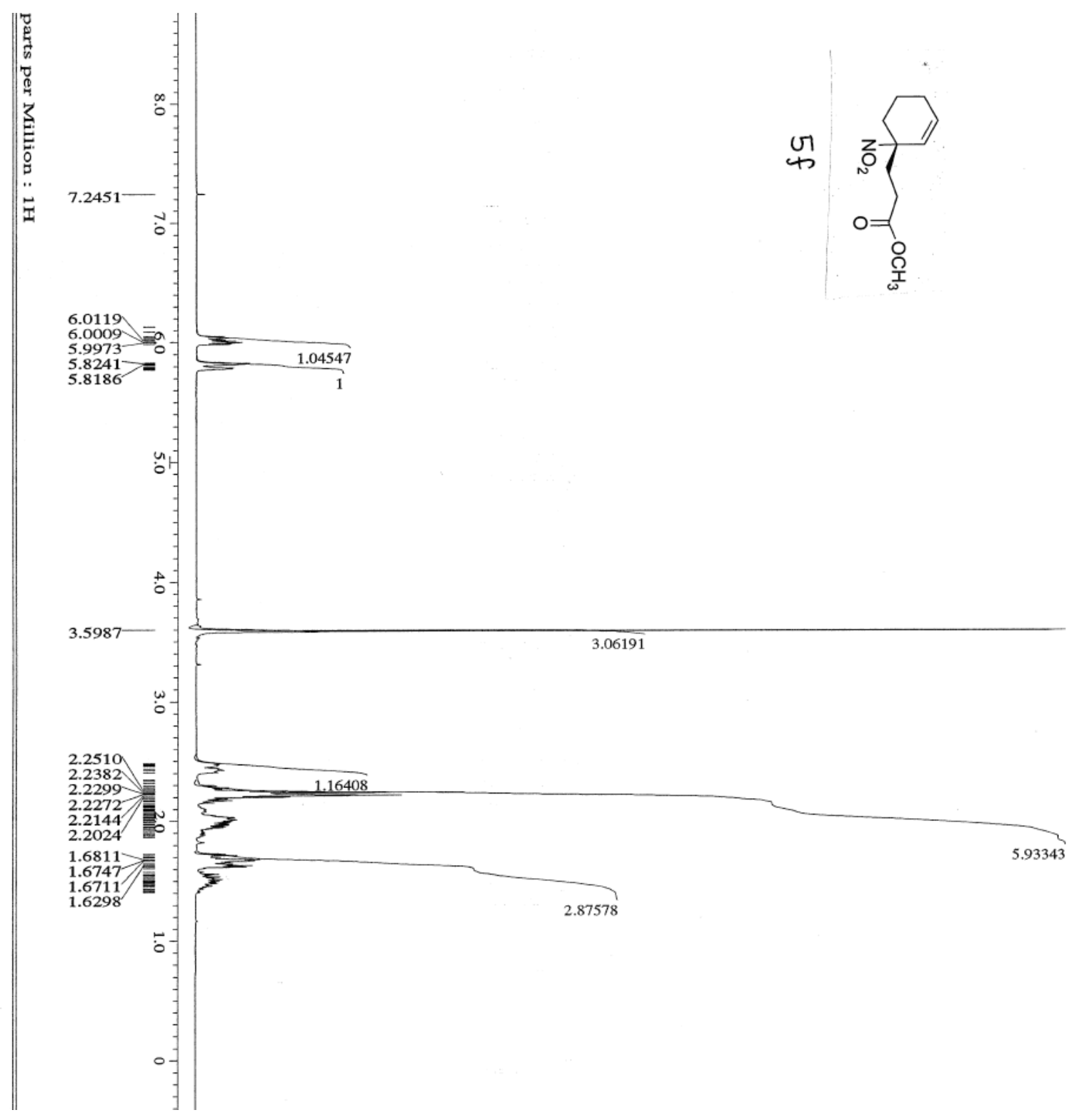

S-31 


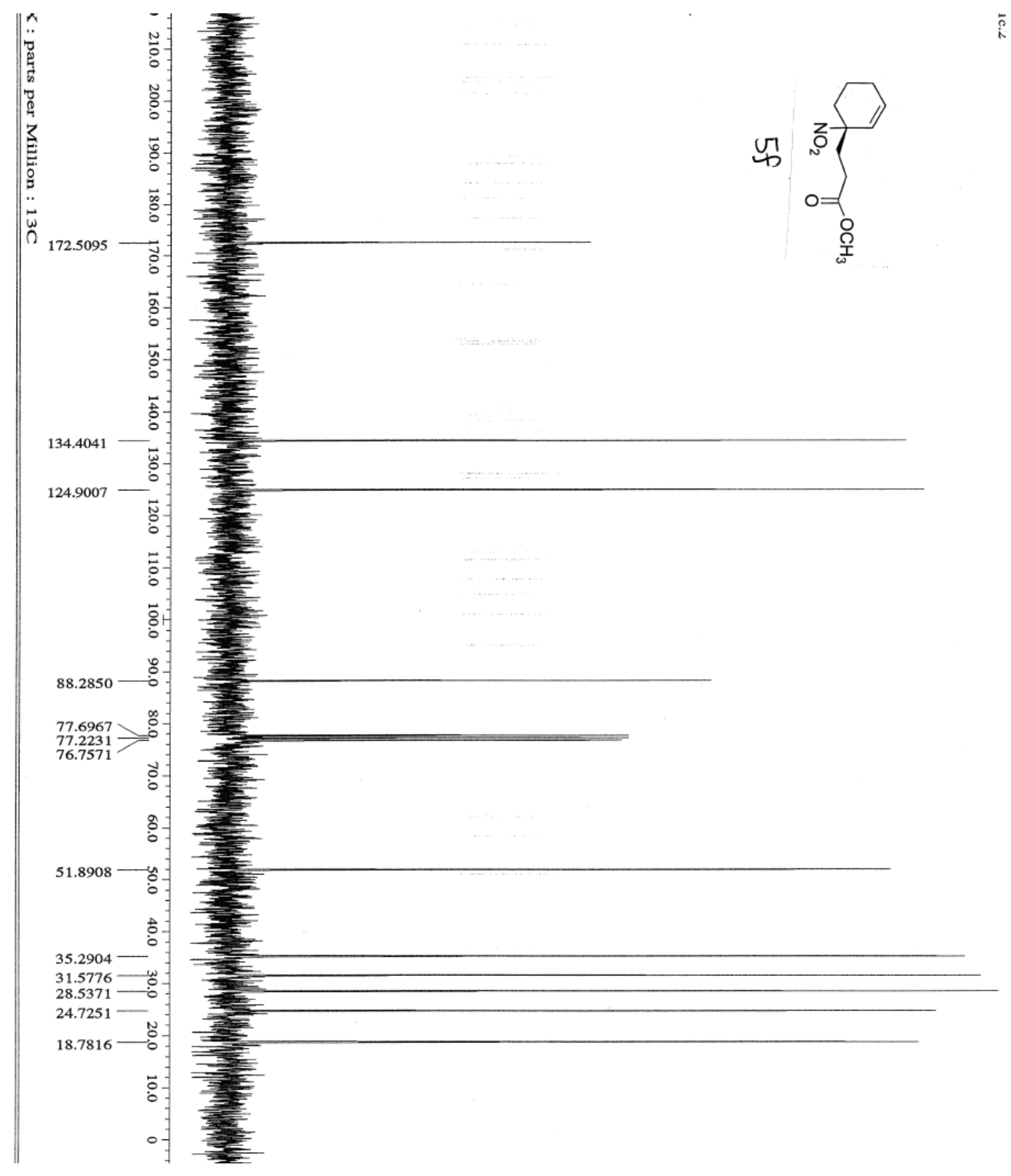




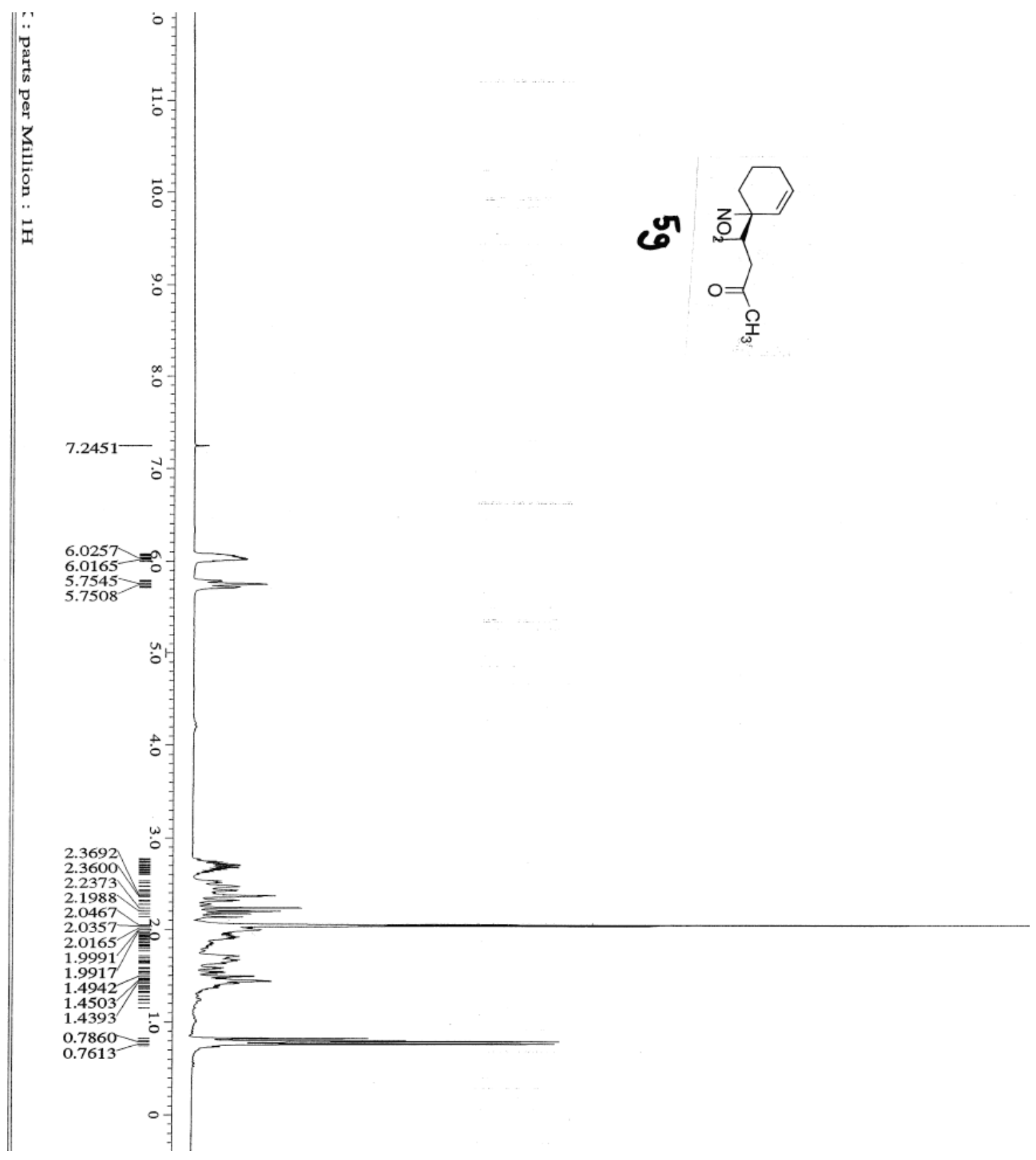

S-33 


$$
\text { l }
$$




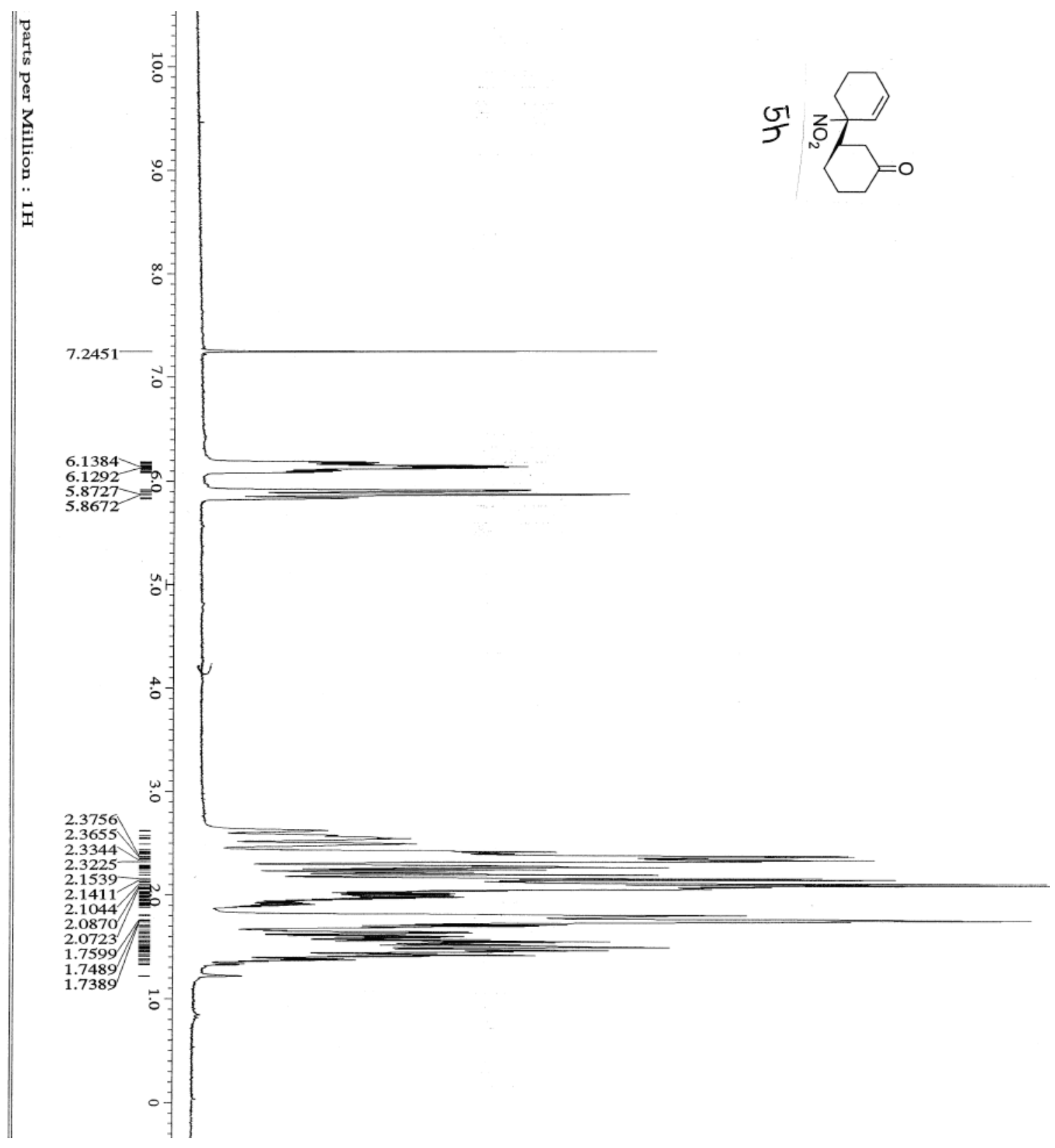




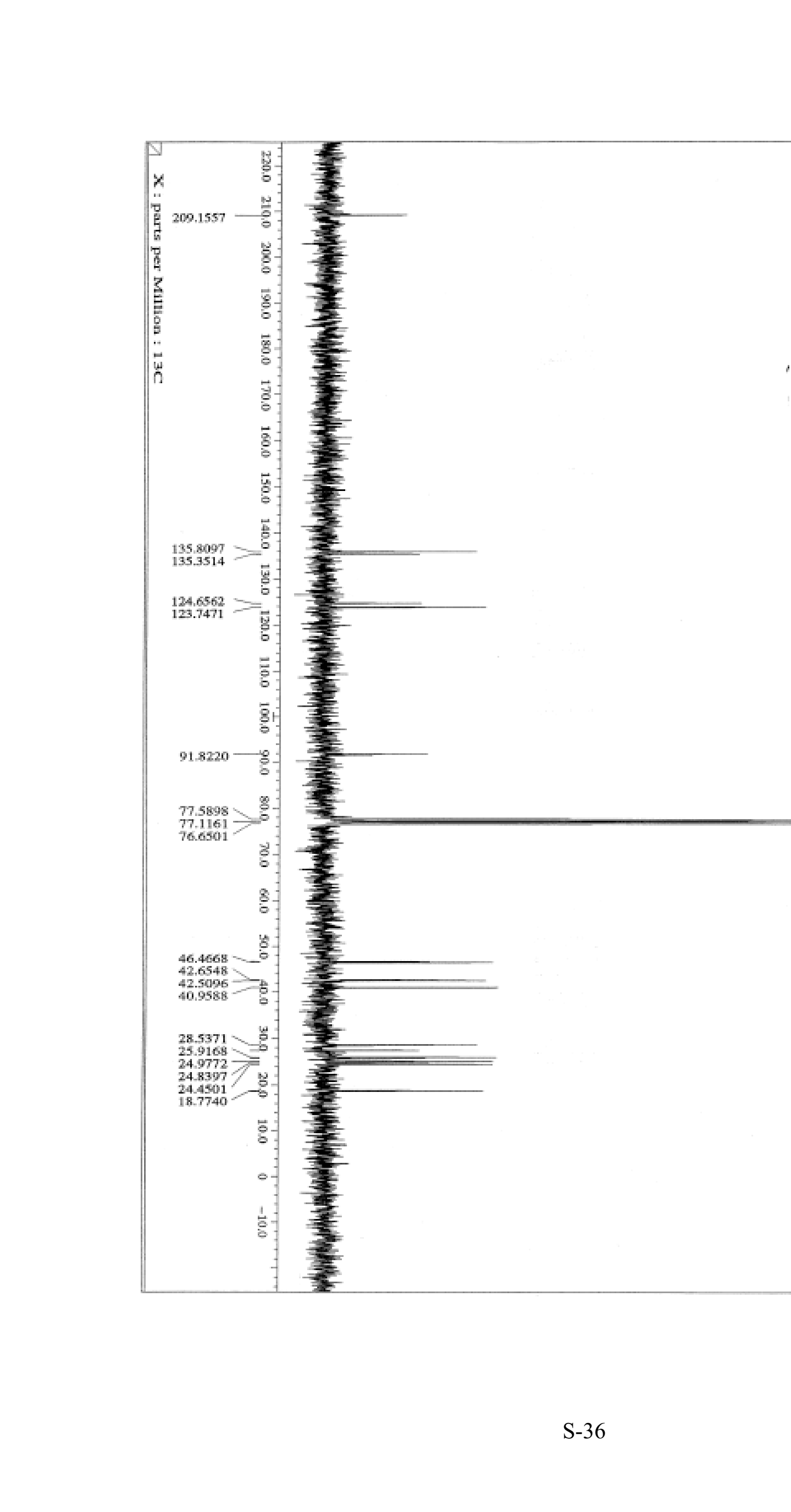




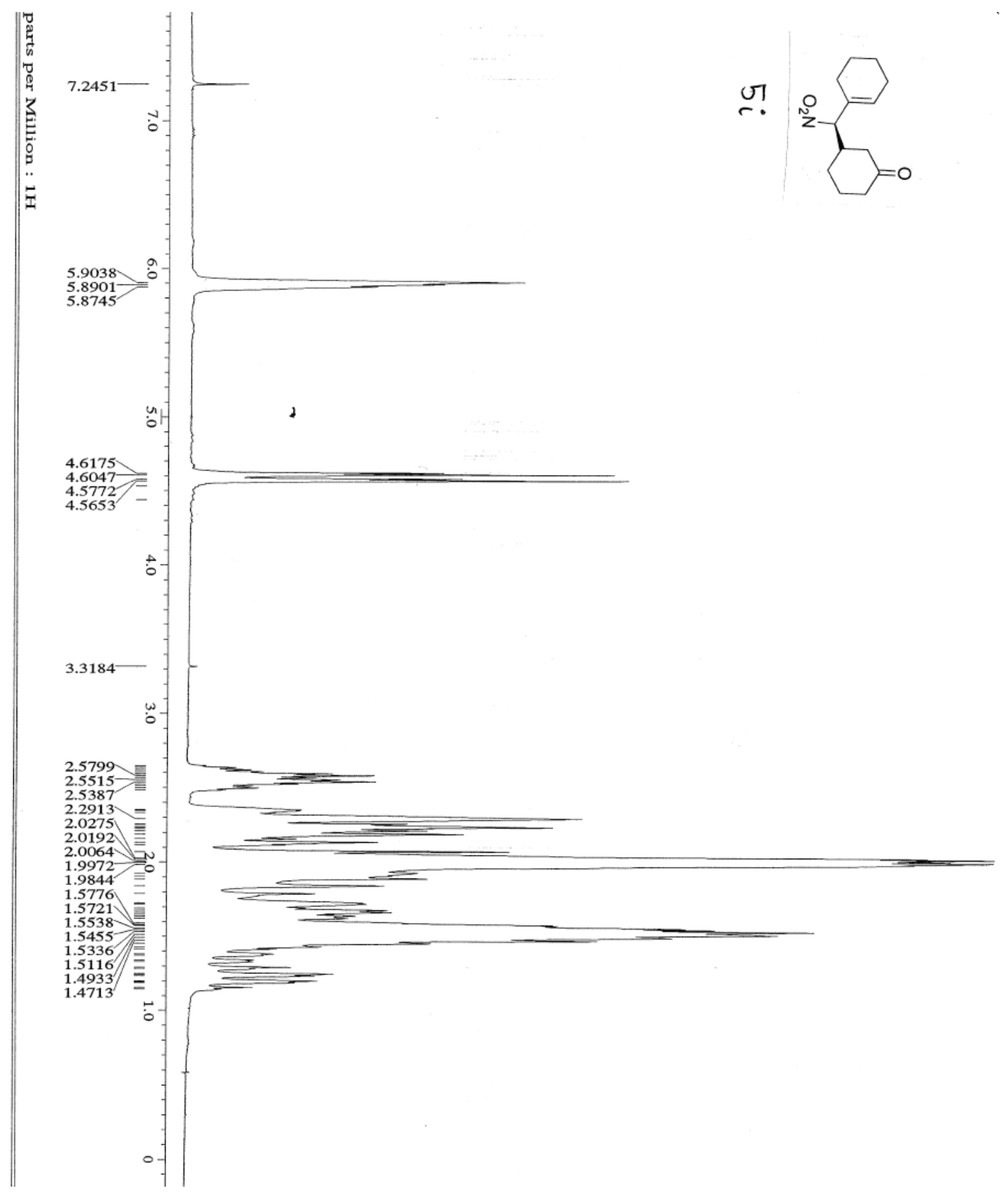




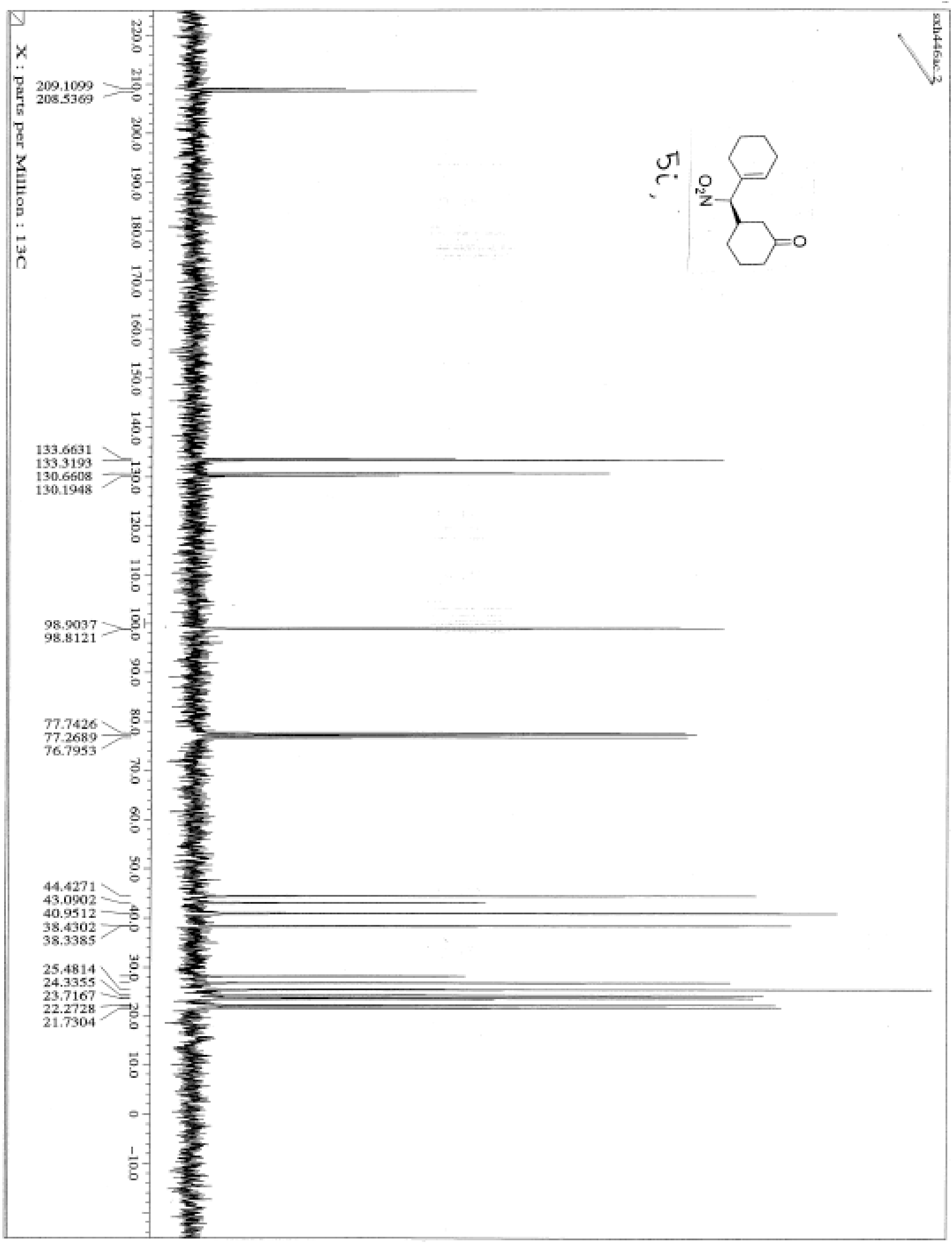




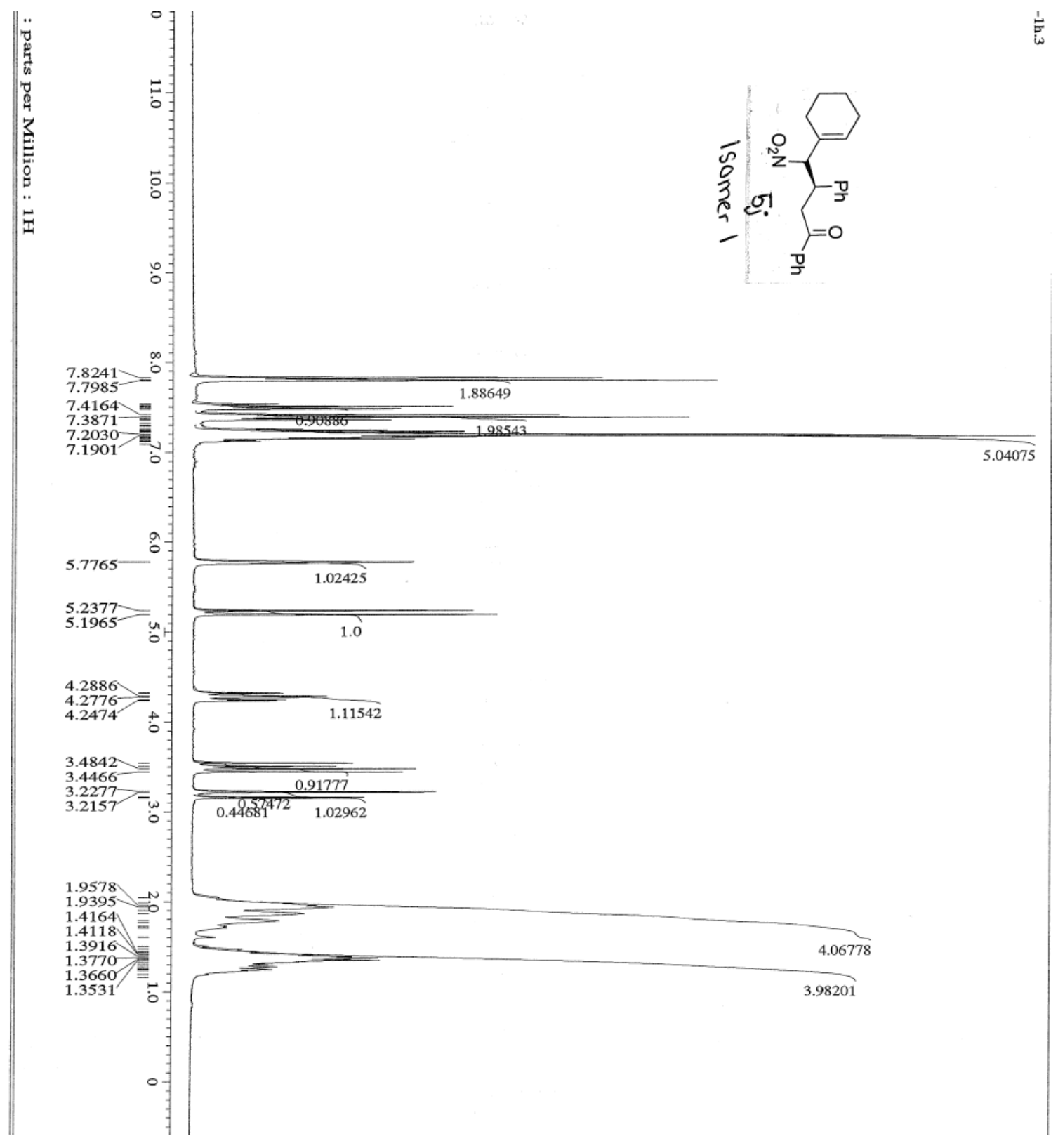




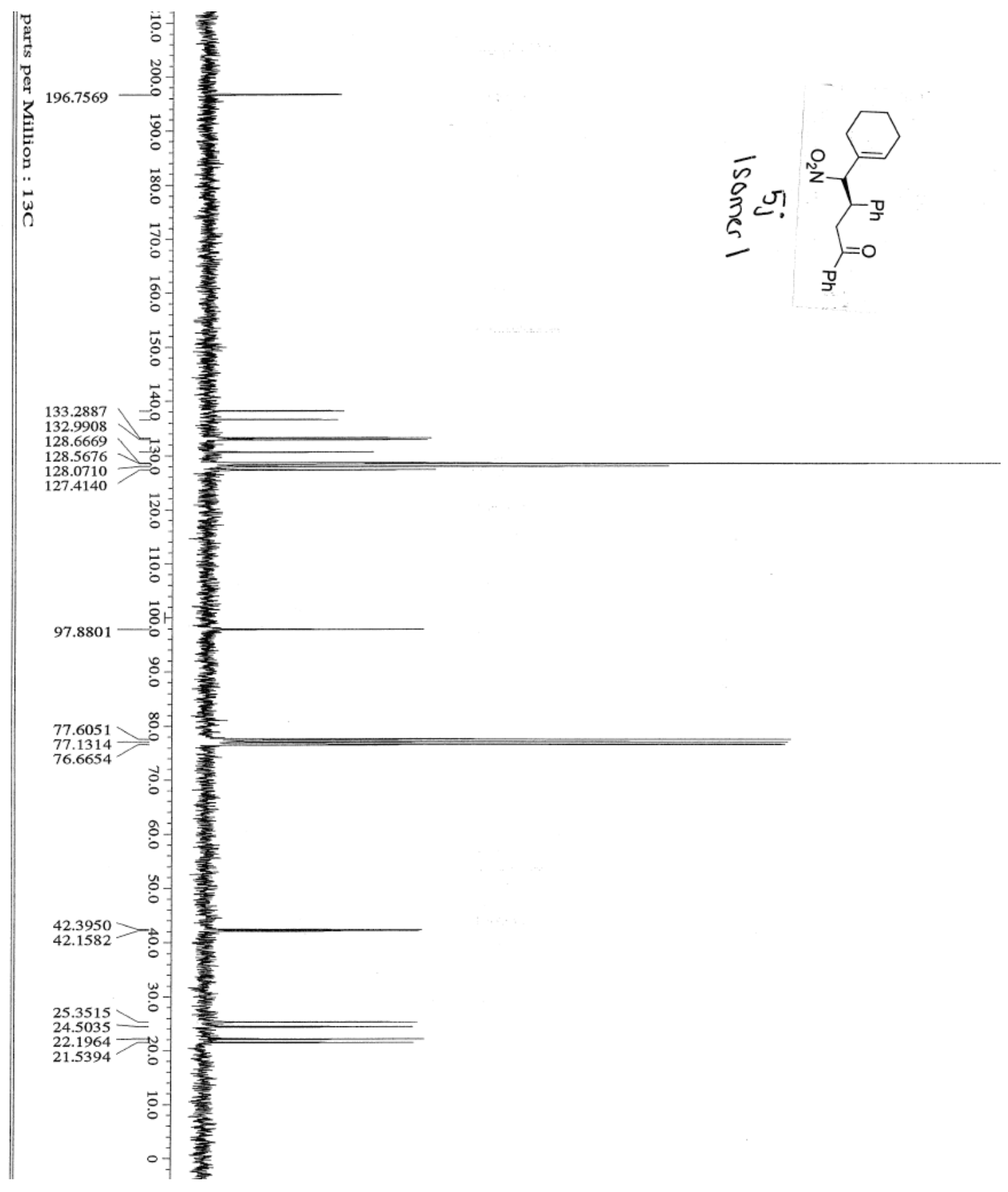




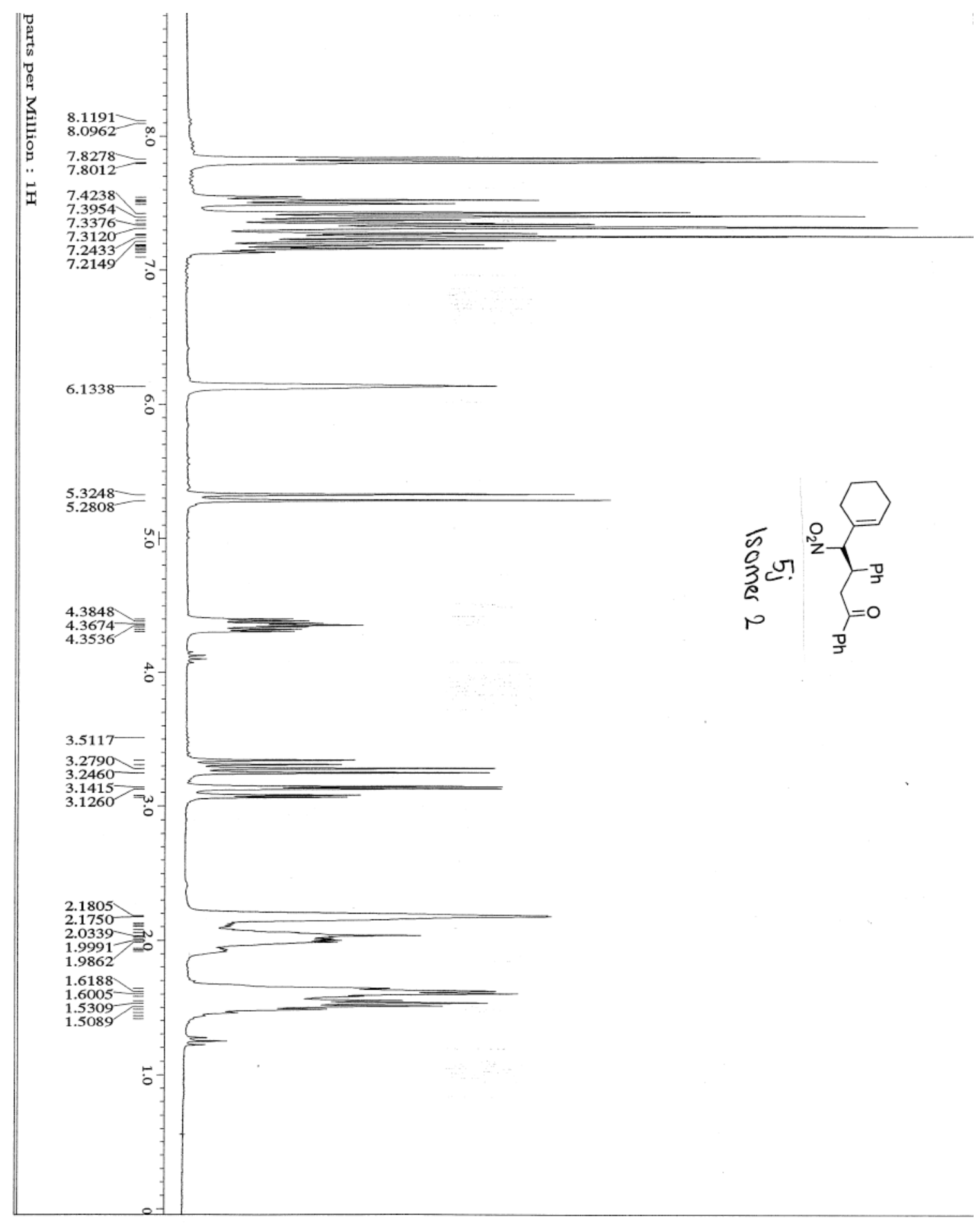




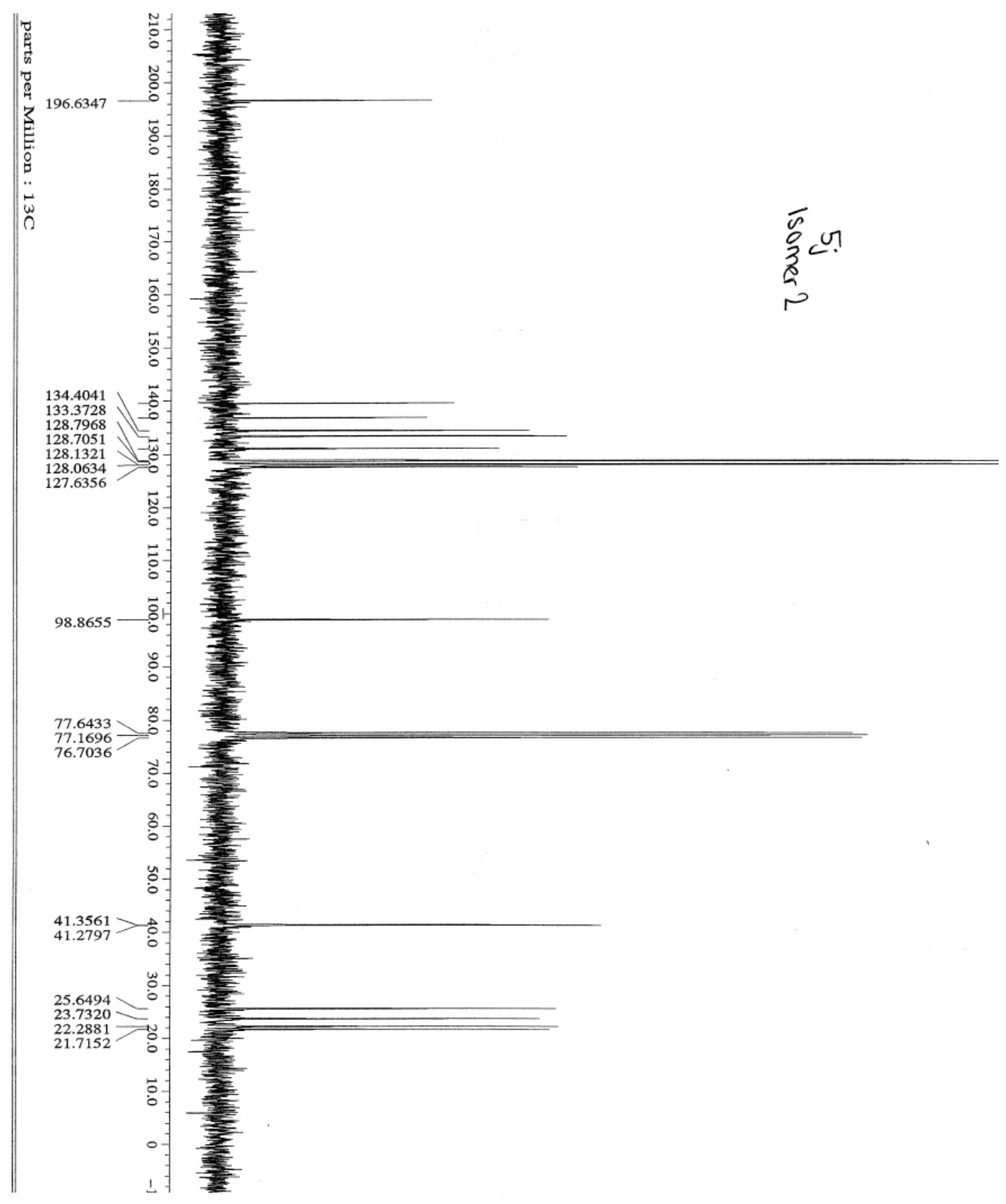




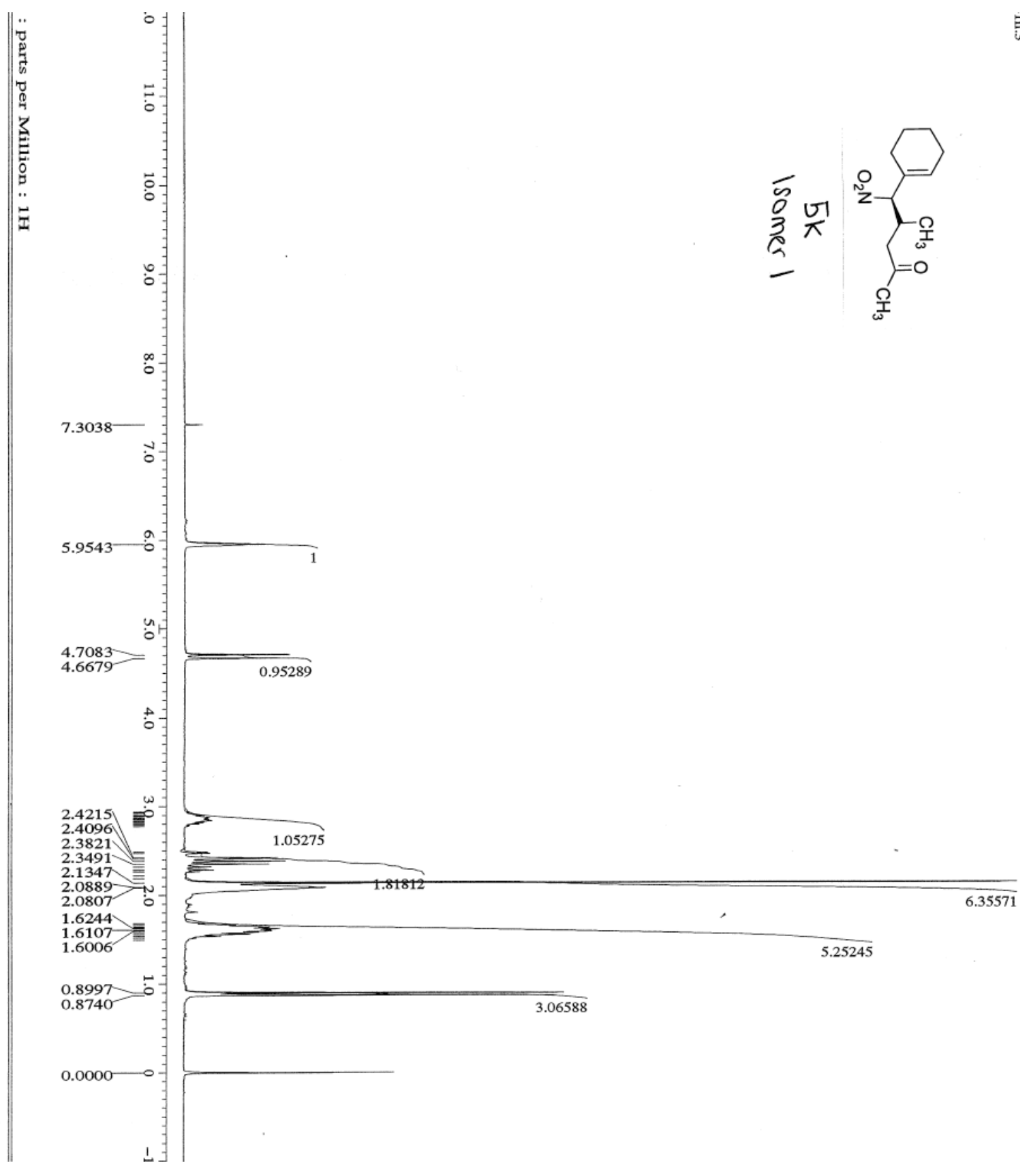




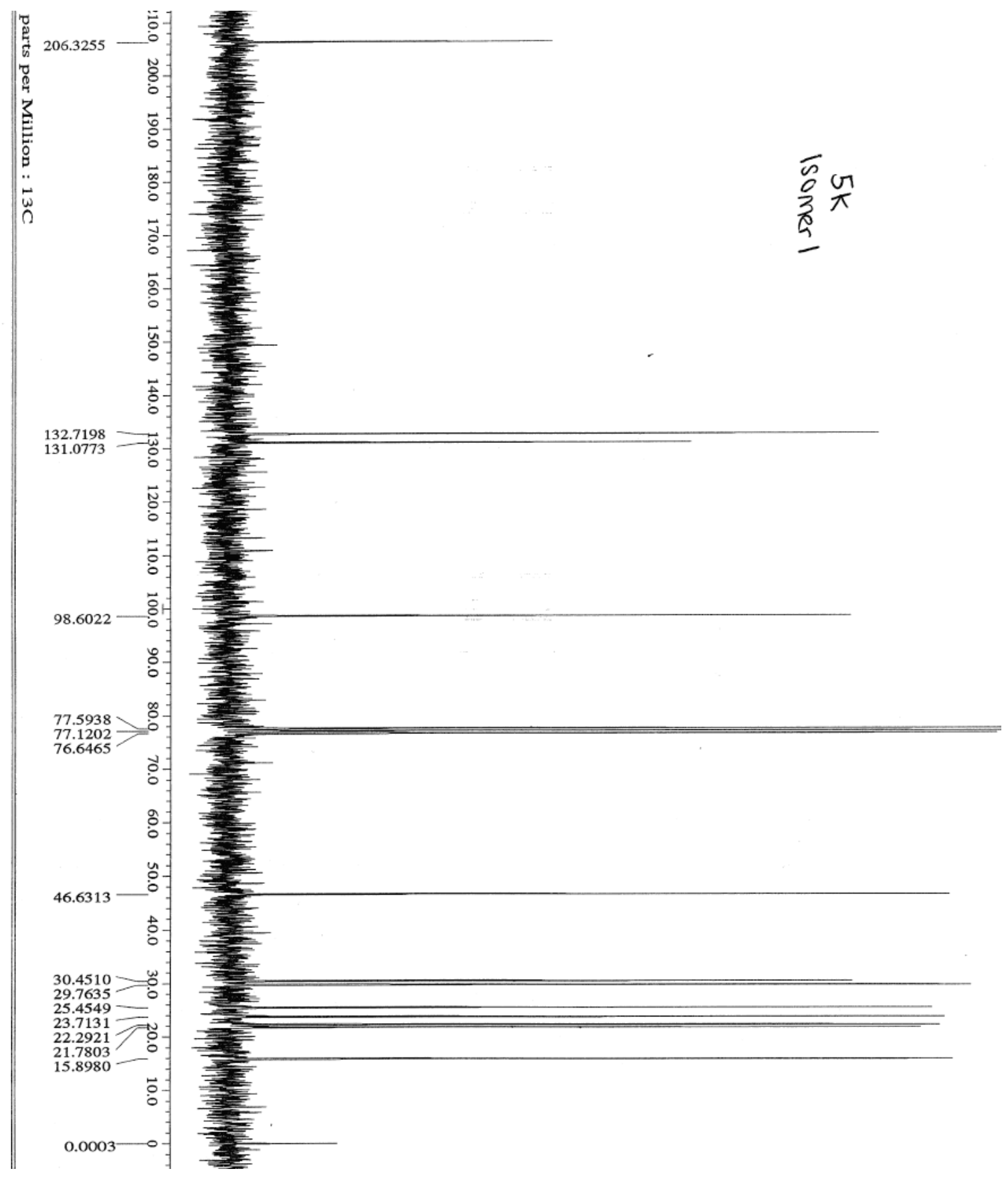




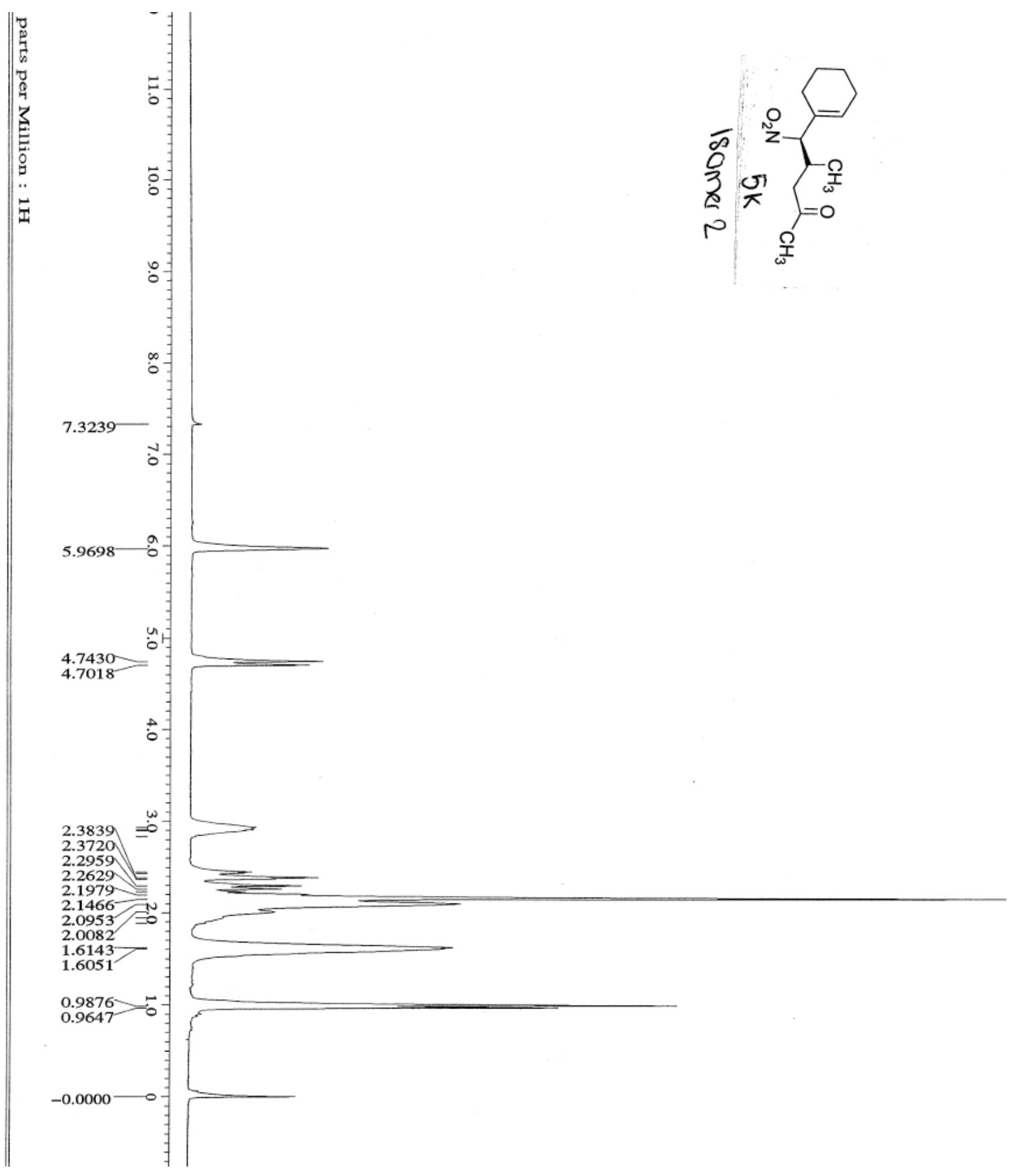




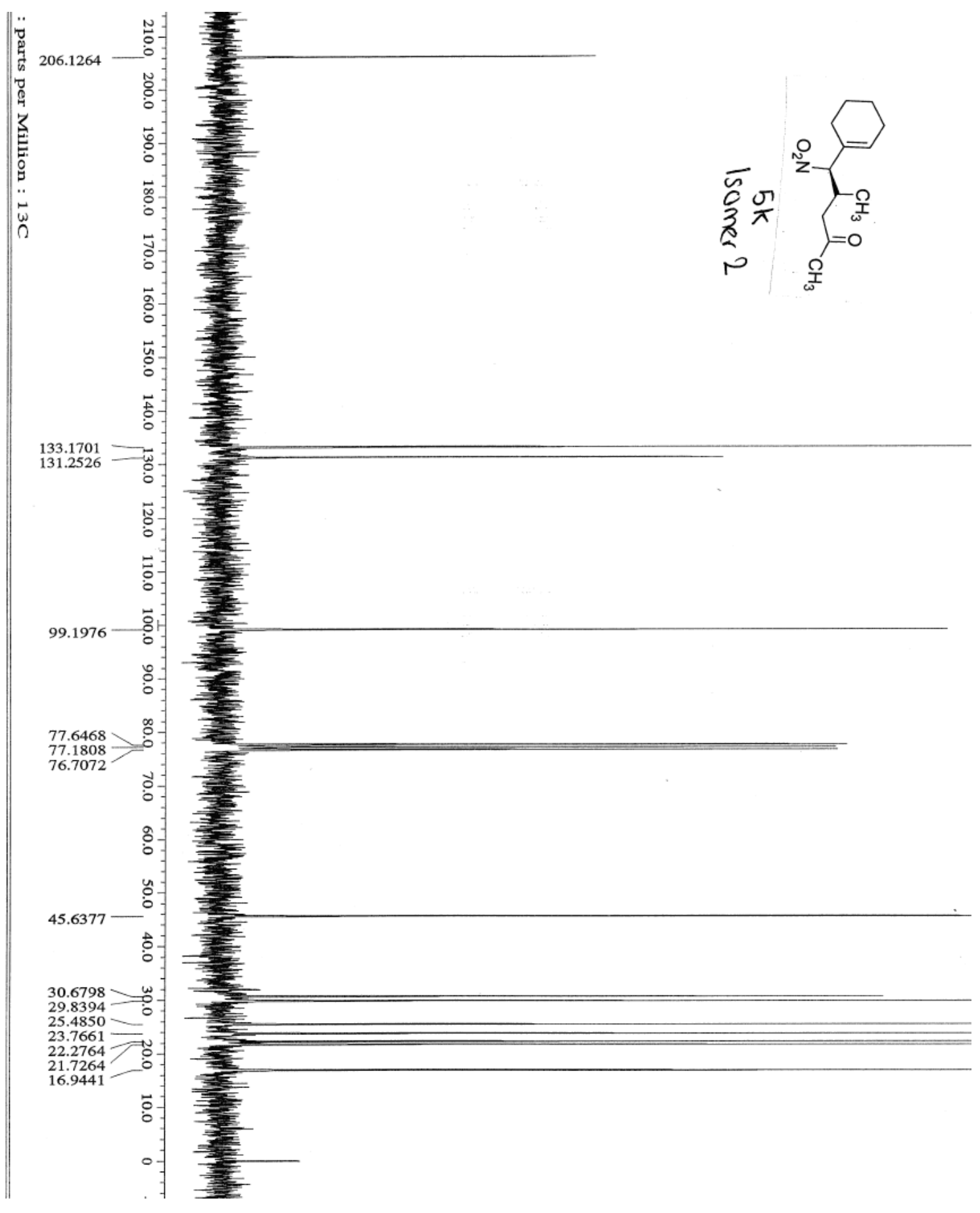




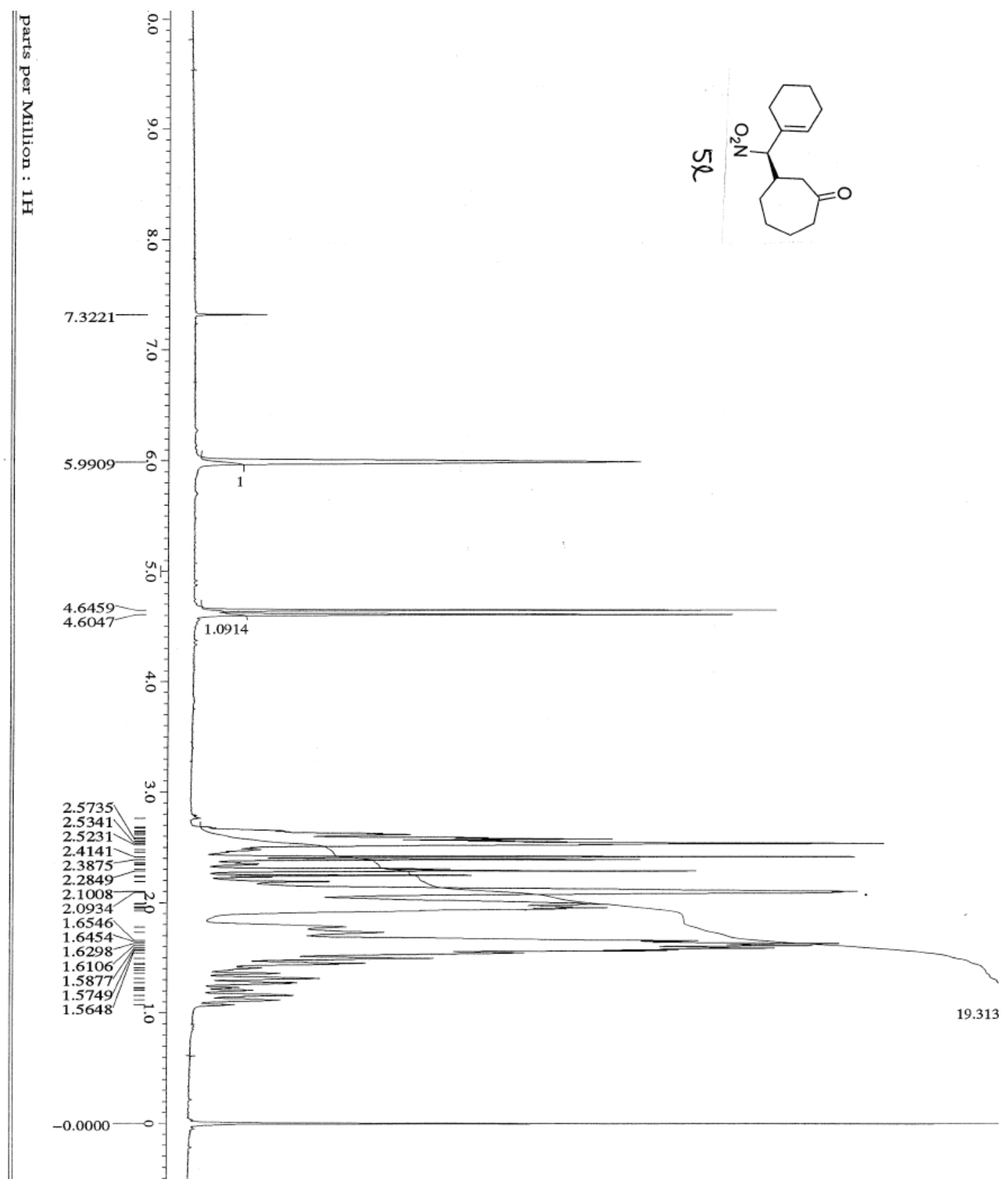




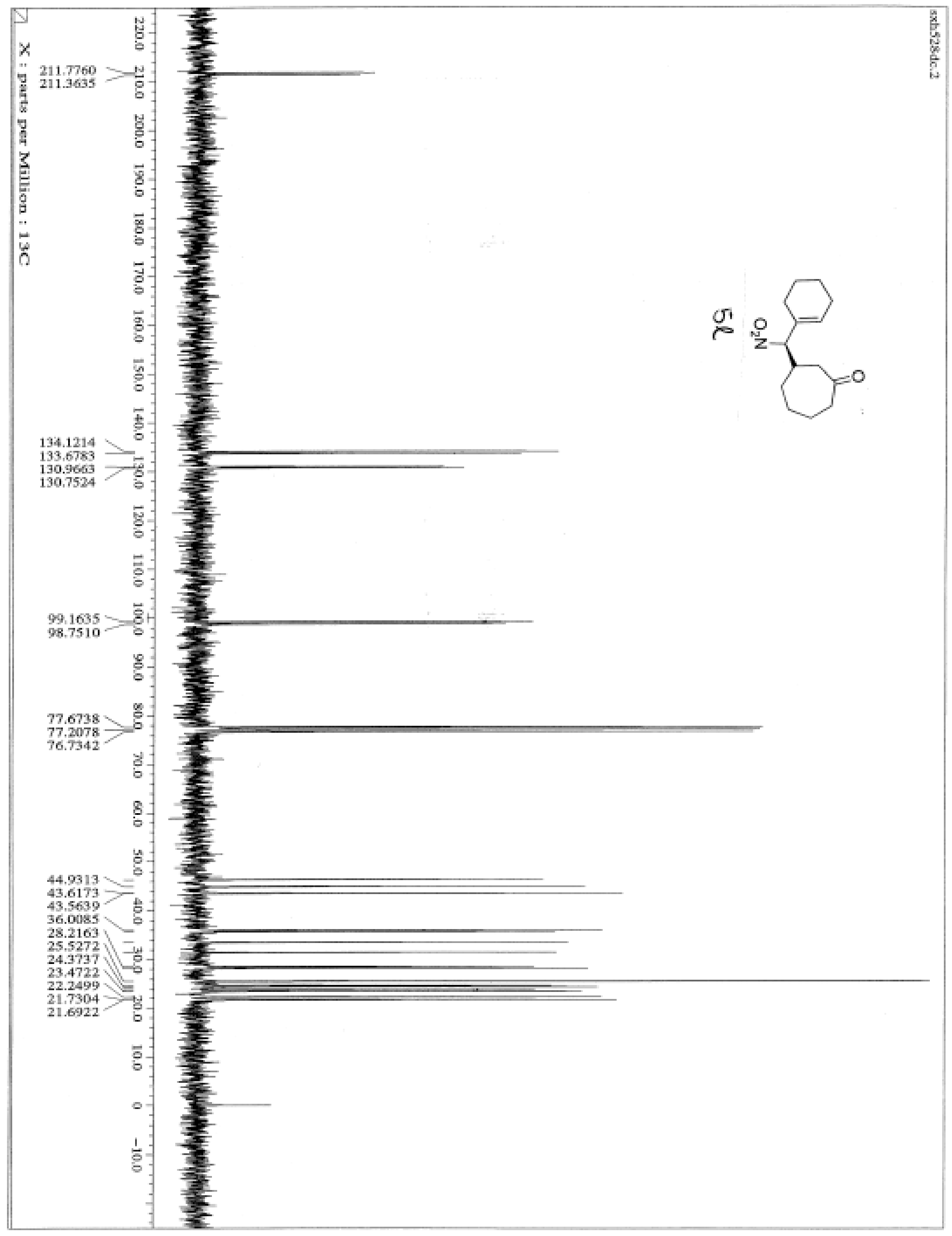




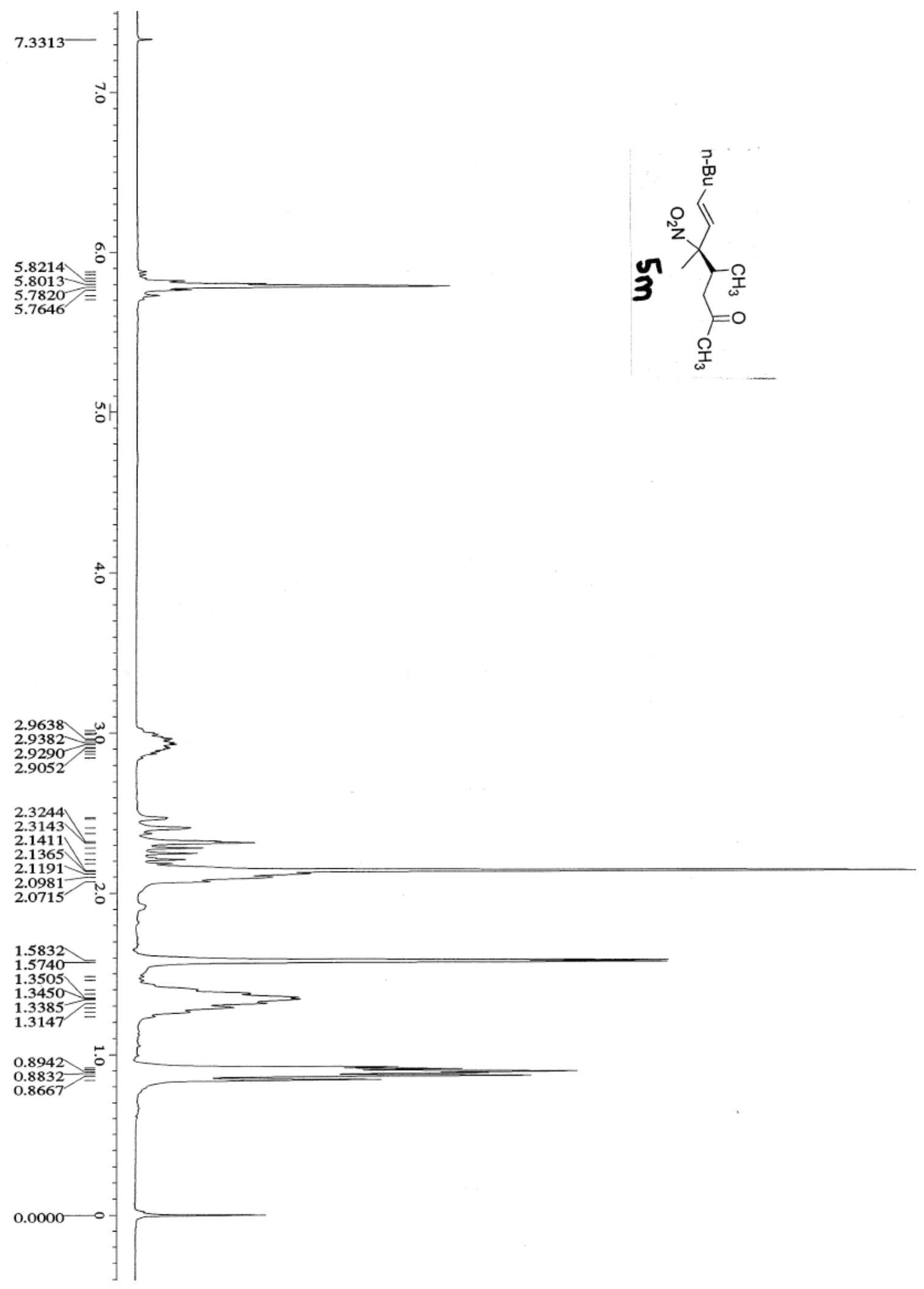




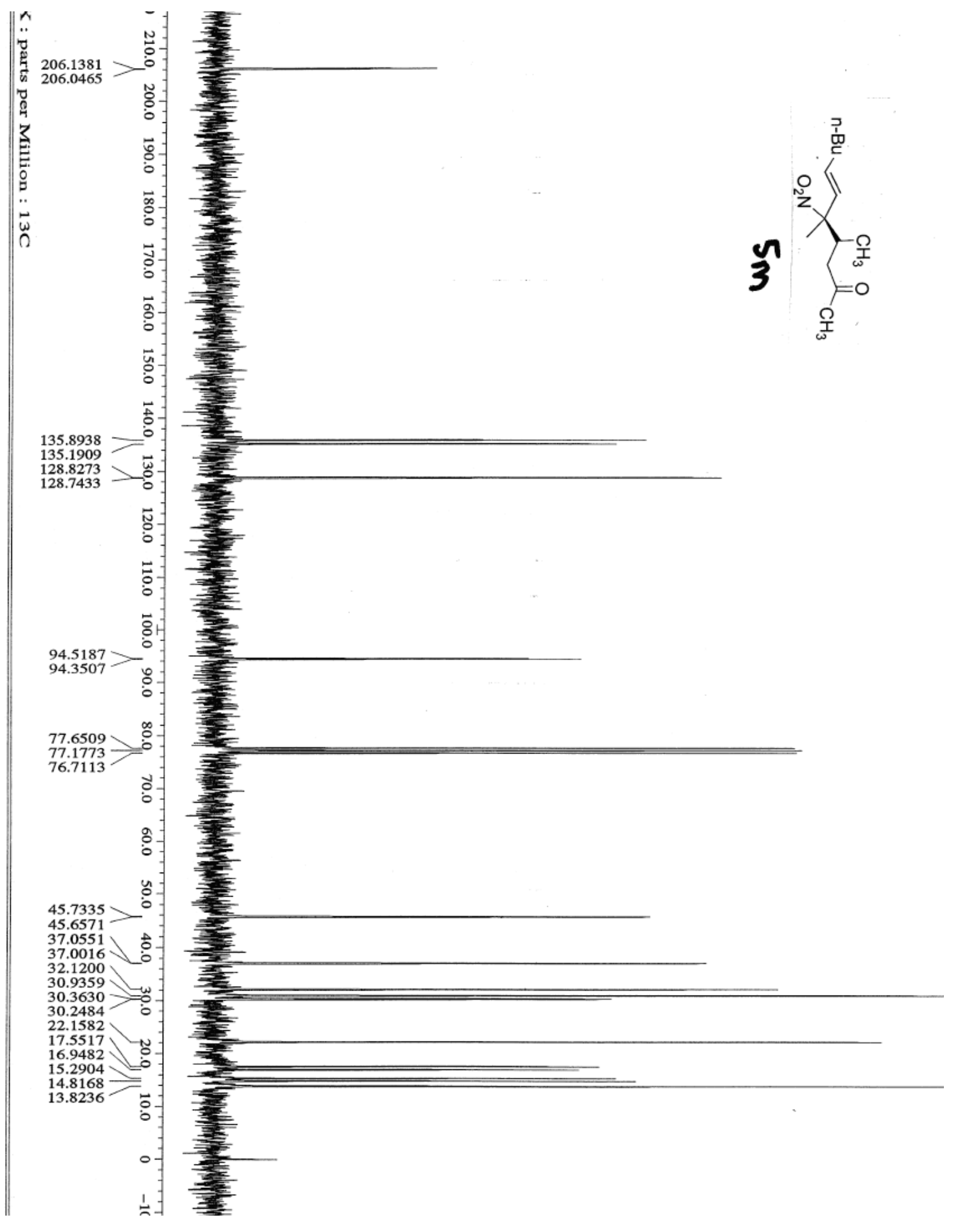




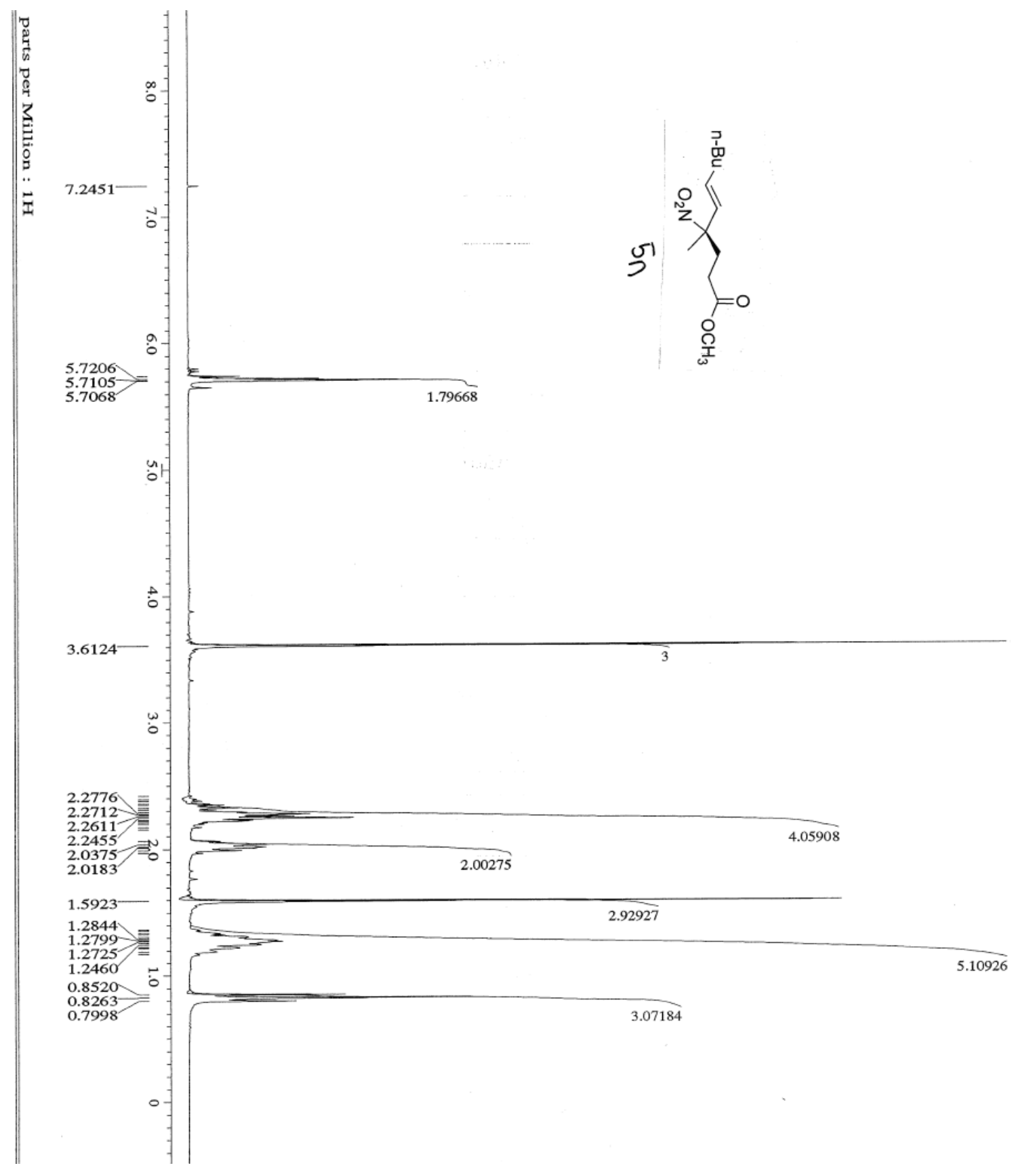




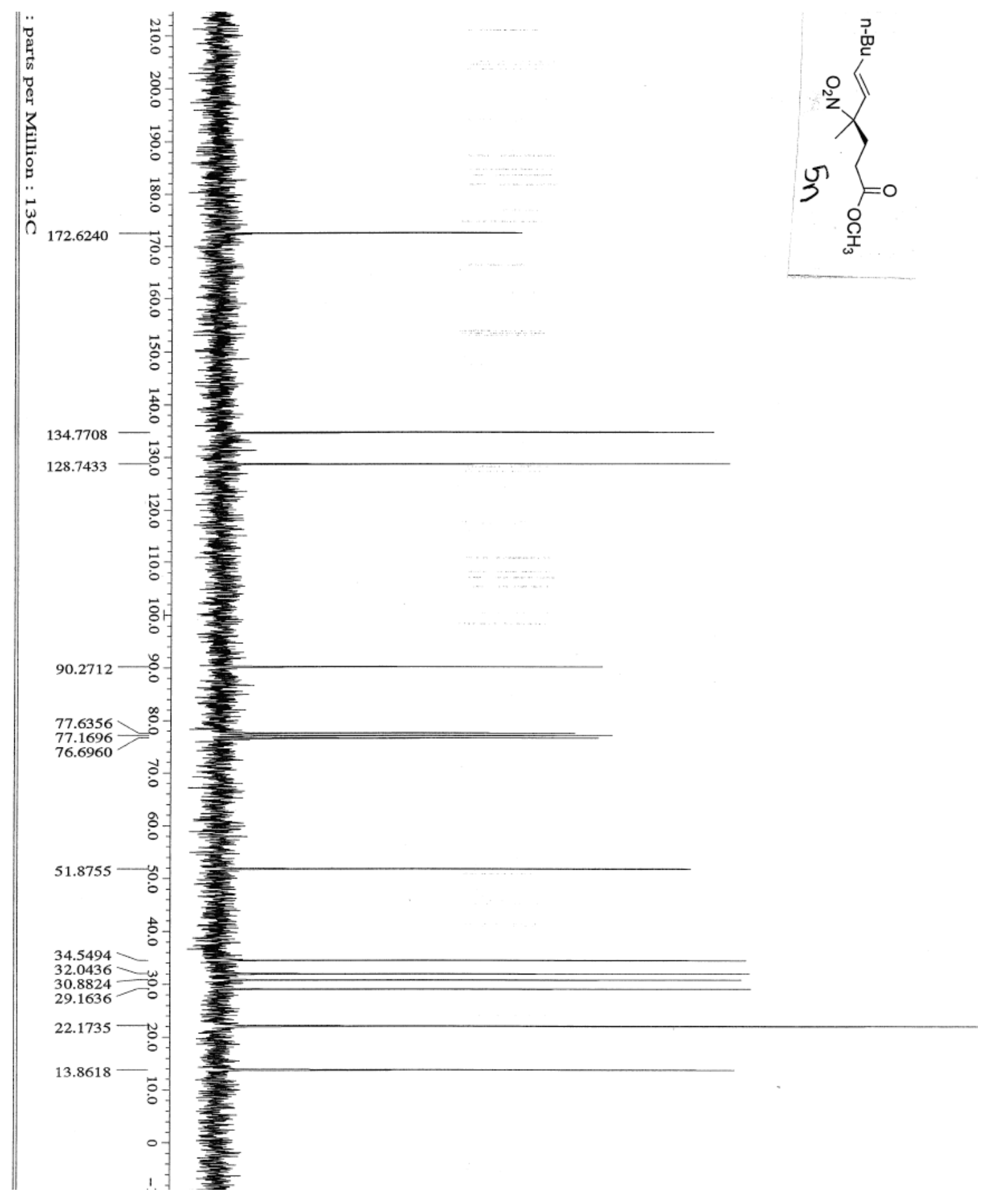




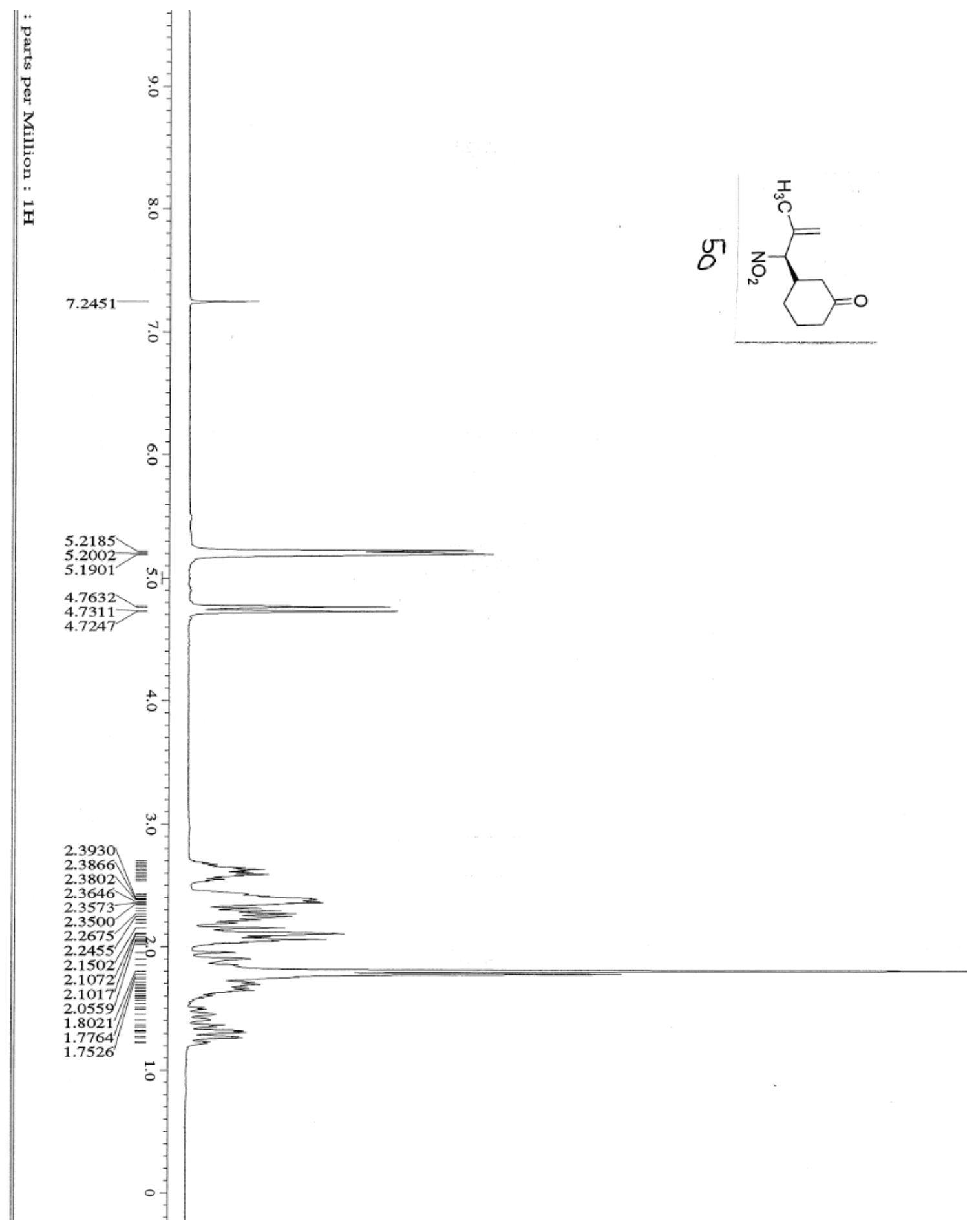




$$
1
$$




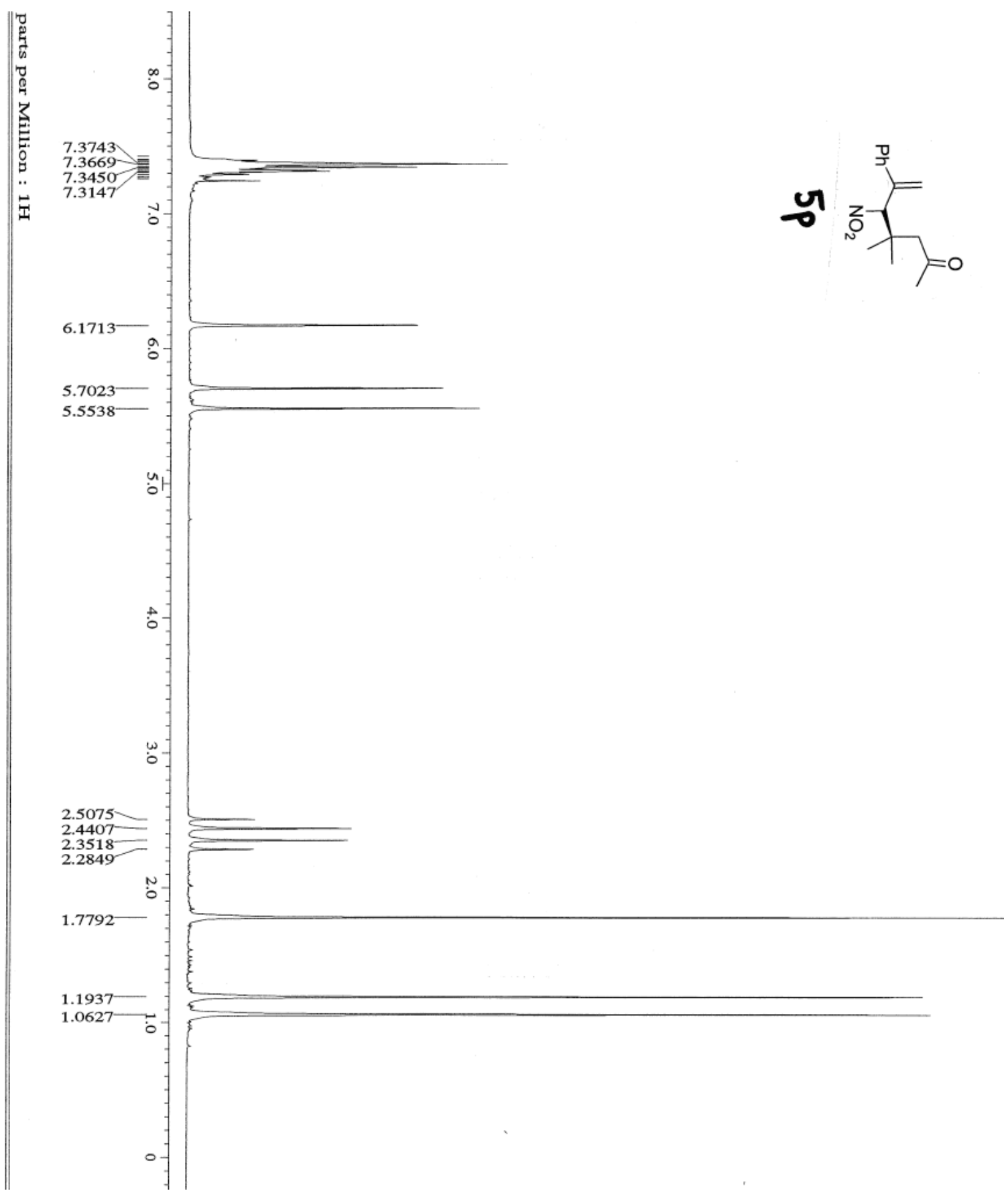




$$
\text { H }
$$




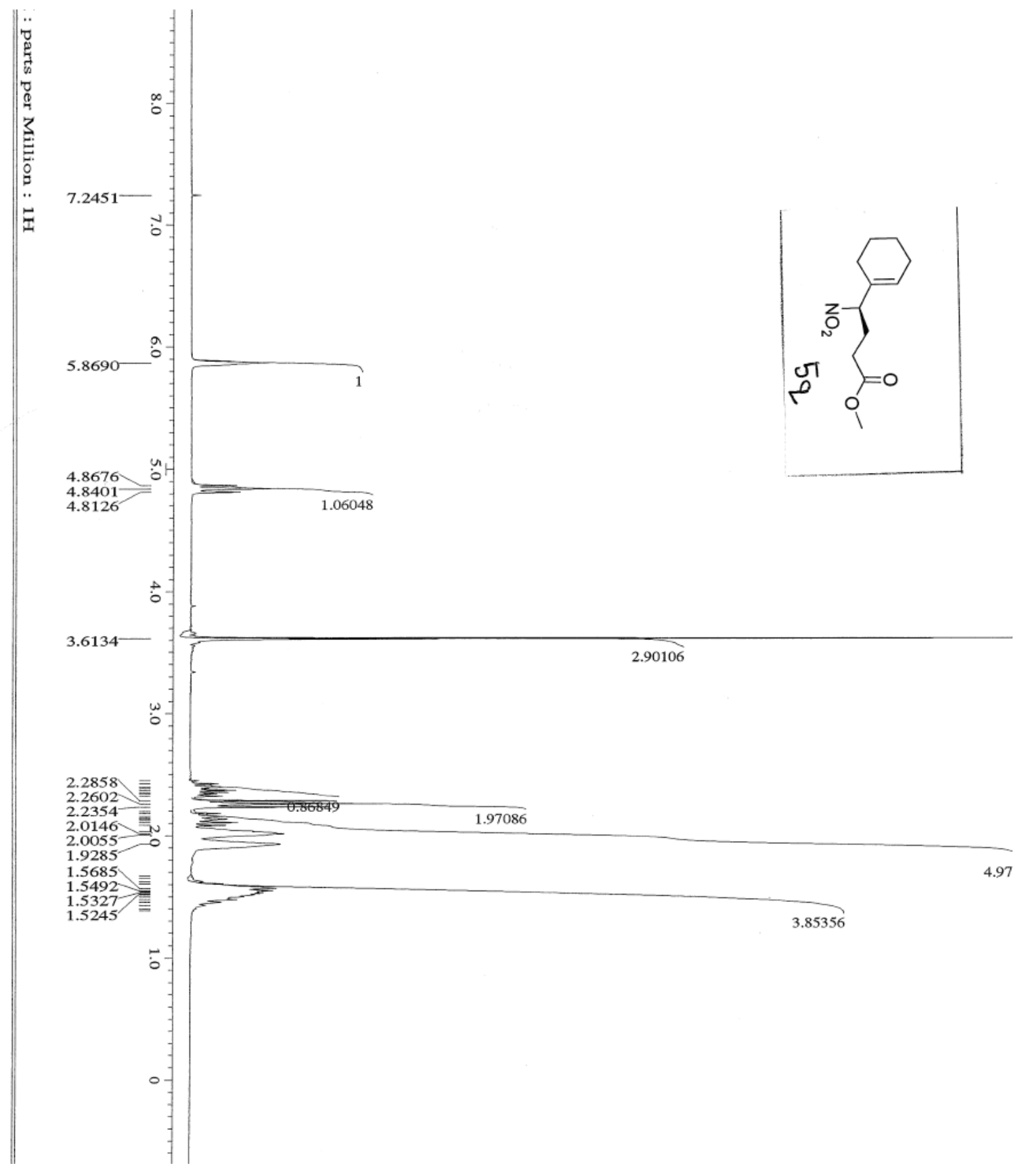




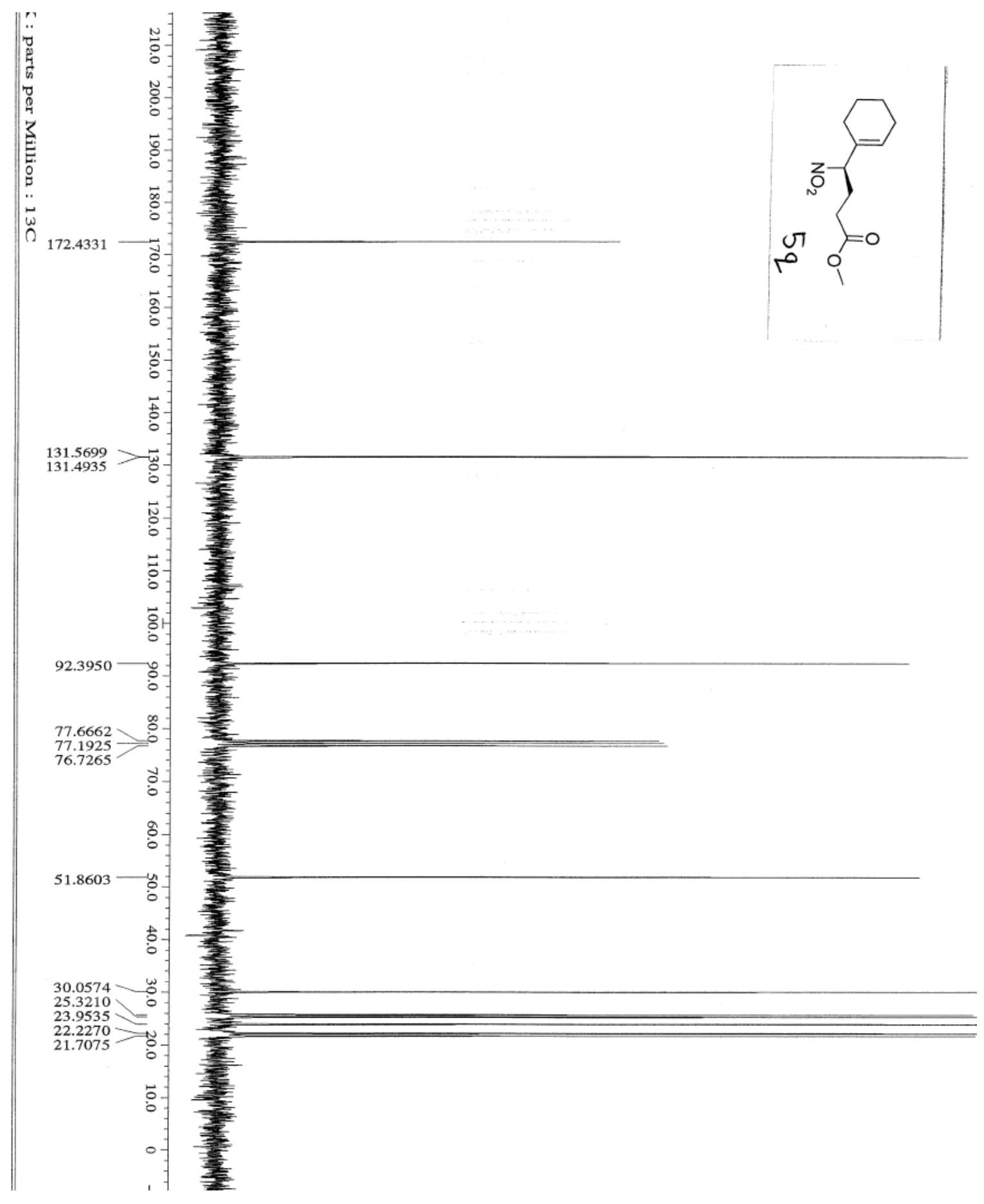




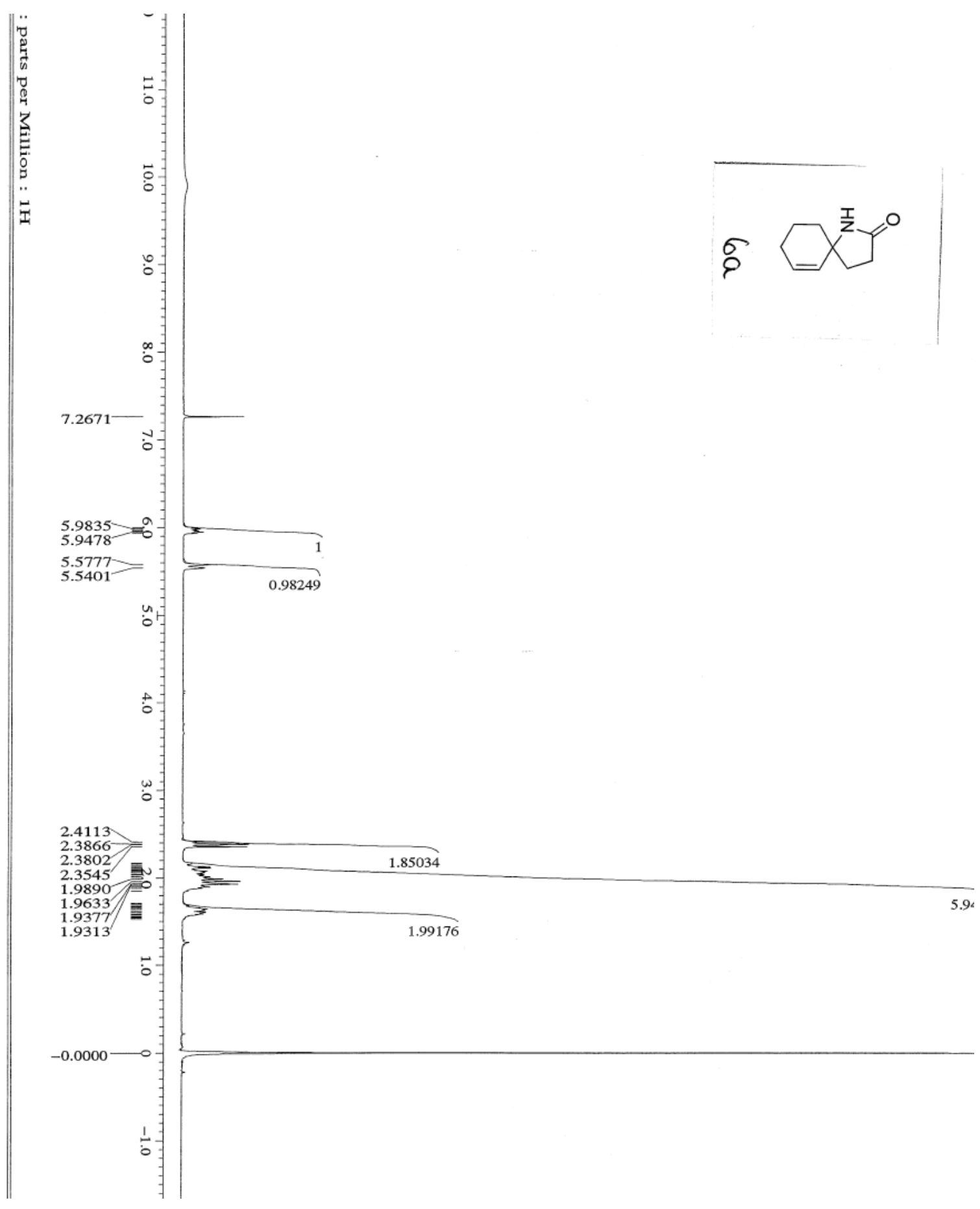




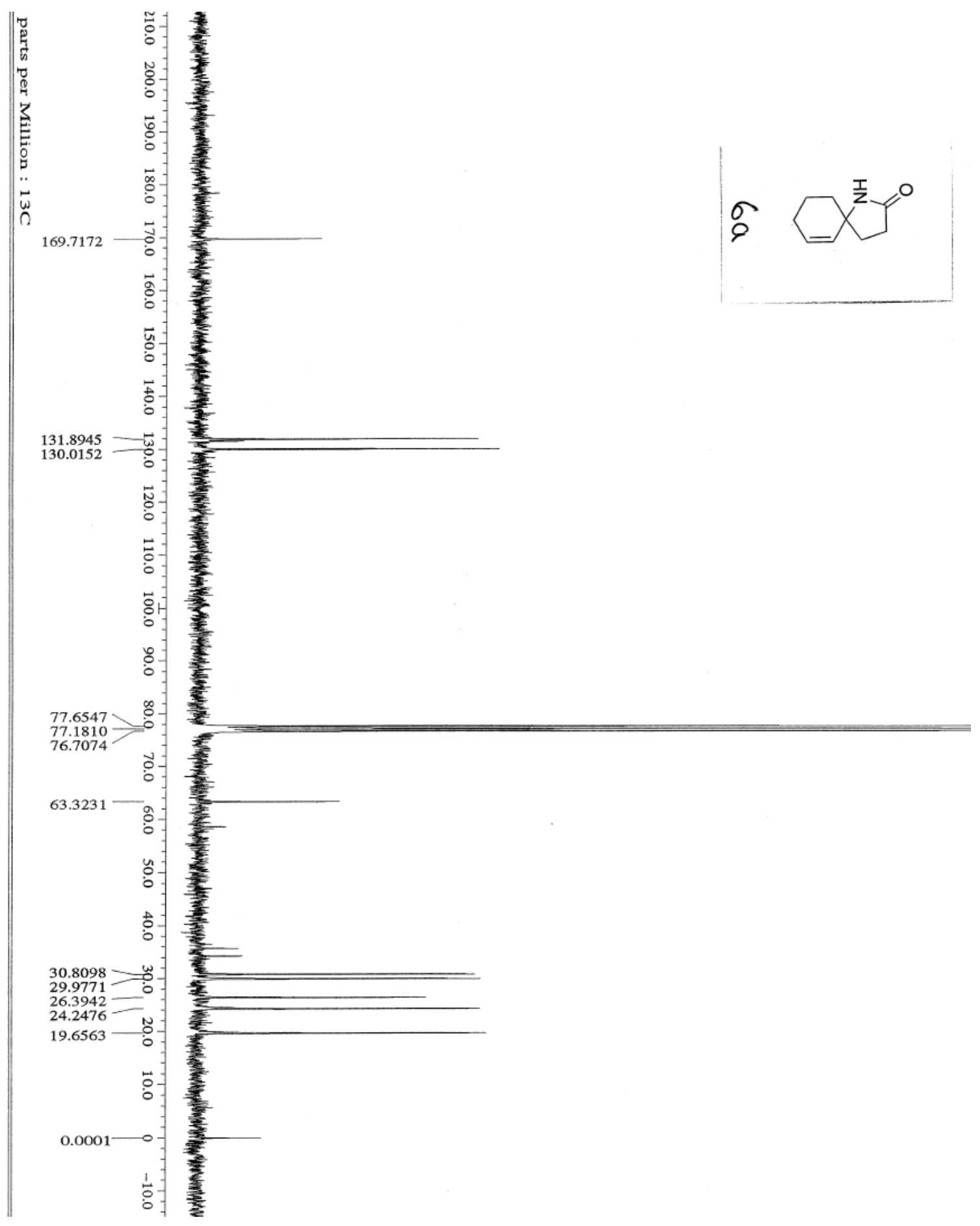




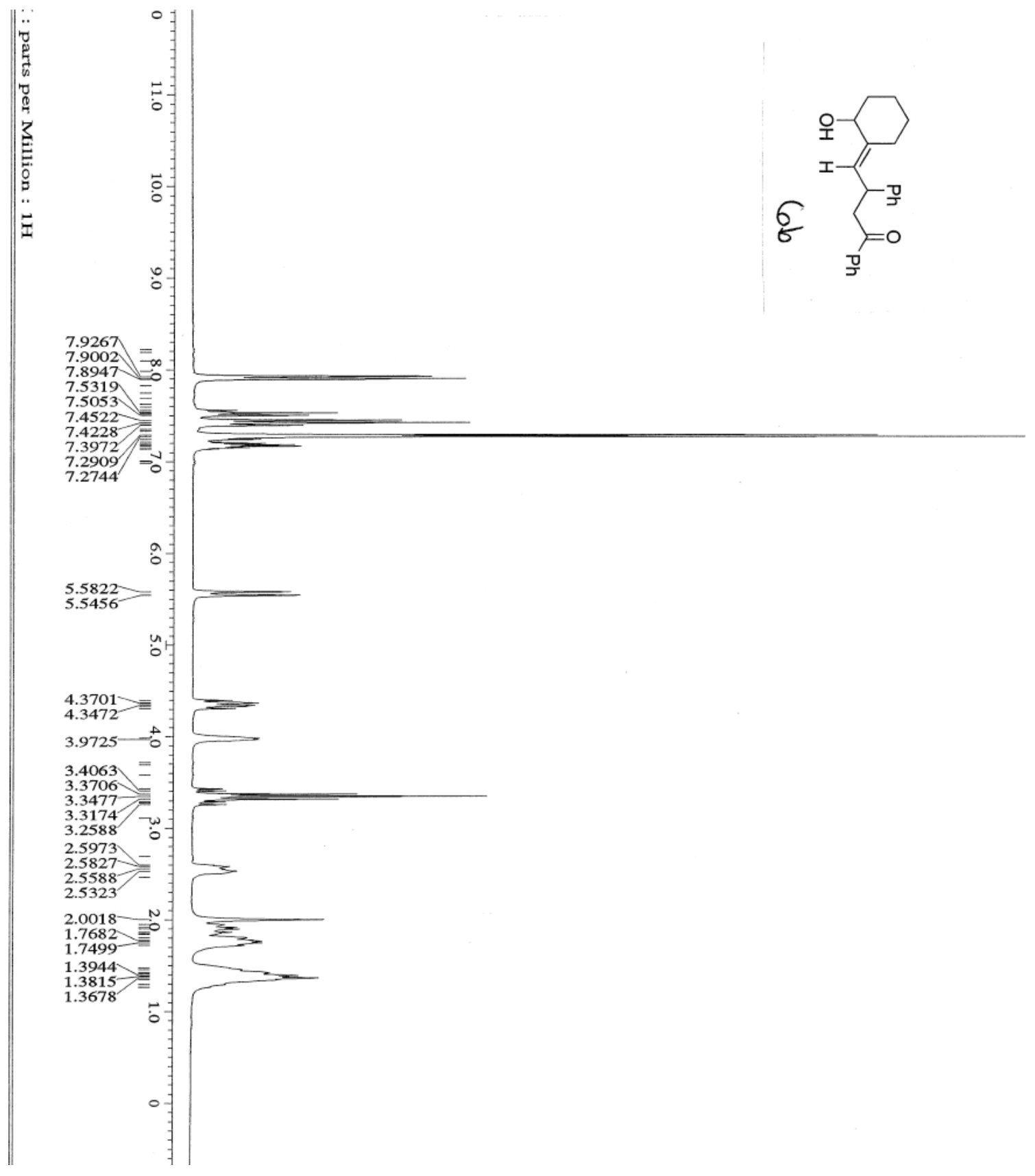




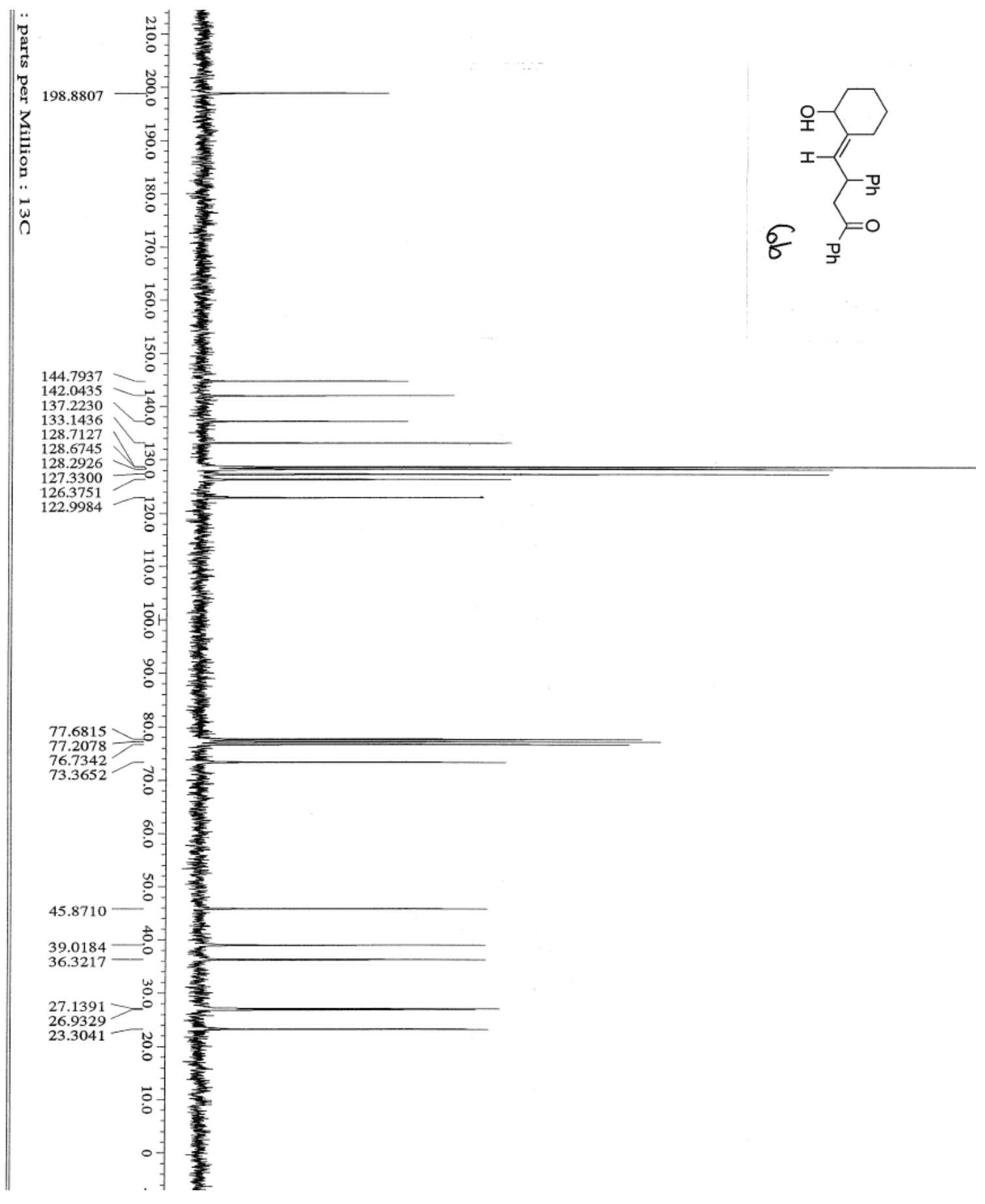




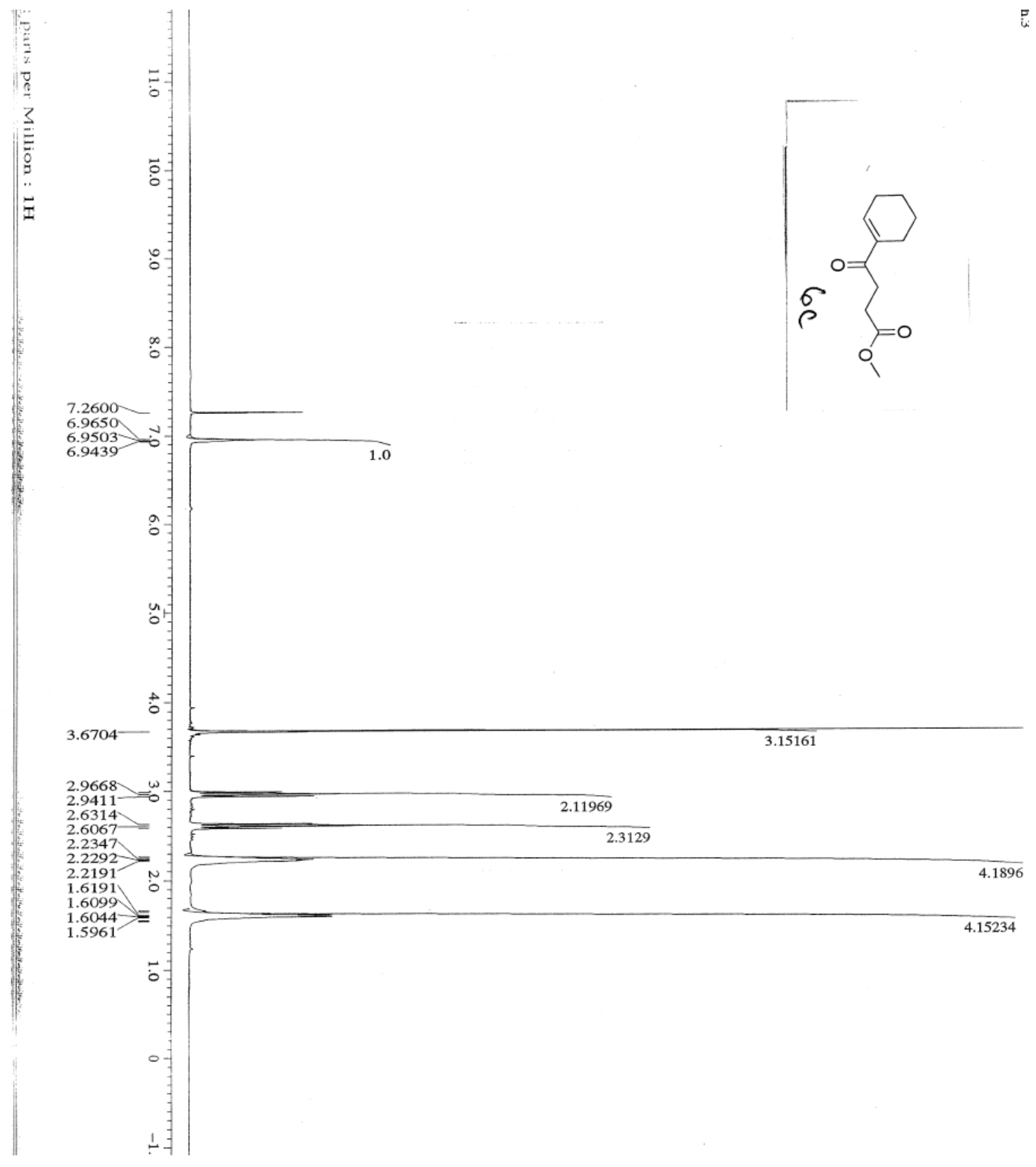




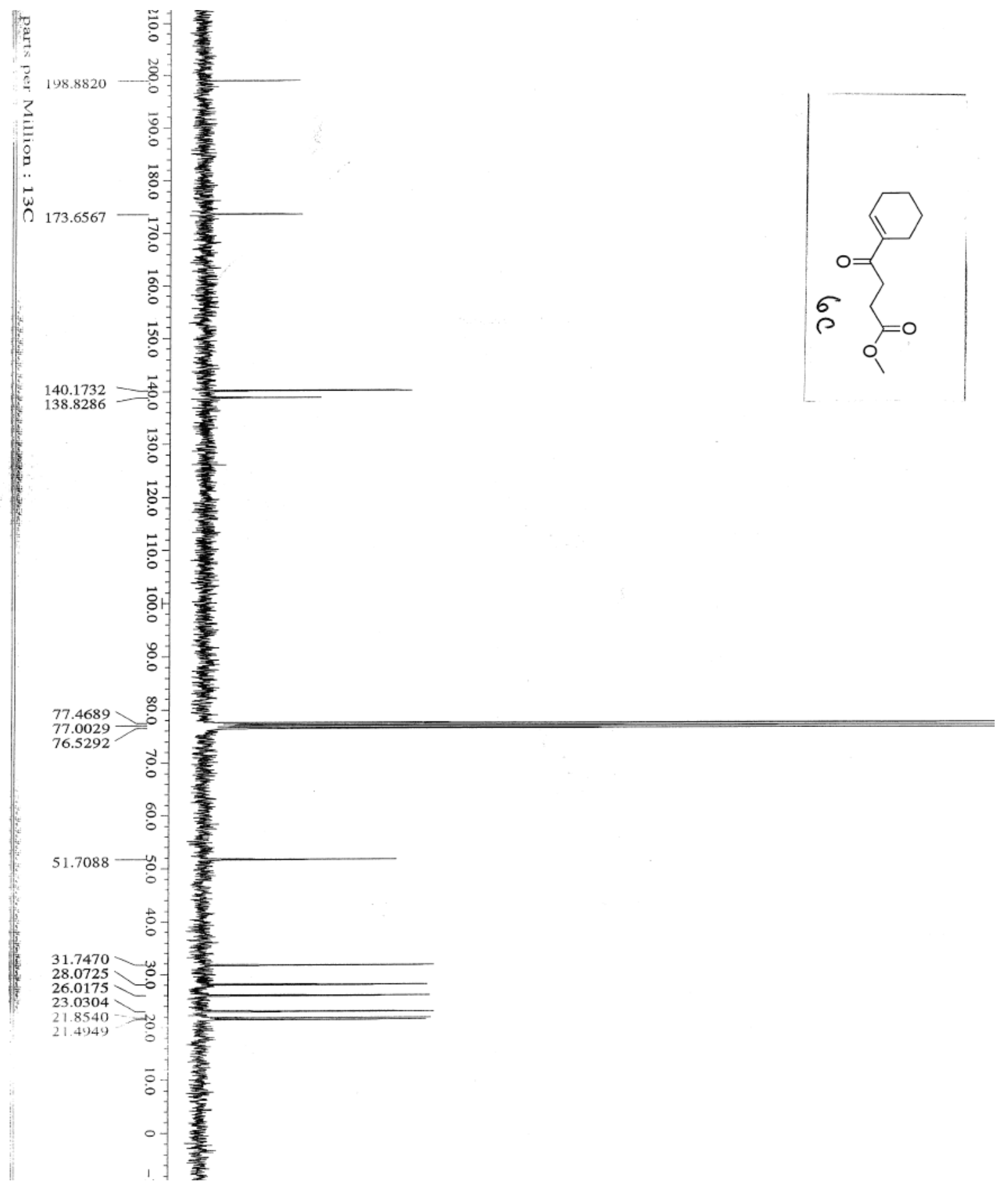

S-64 